Y. Namikawa

Nagoya Math. J.

Vol. 52 (1973), 197-259

\title{
ON THE CANONICAL HOLOMORPHIC MAP FROM THE MODULI SPACE OF STABLE CURVES TO THE IGUSA MONOIDAL TRANSFORM*)
}

\author{
YUKIHIKO NAMIKAWA
}

\section{Introduction.}

Let $\mathscr{M}_{g}$ be the coarse moduli space of complete non-singular curves of genus $g$ and $\widetilde{S}_{g}^{*}$ the coarse moduli space of principally polarized abelian varieties of dimension $g$. There is a canonical map:

$$
i: \mathscr{M}_{g} \rightarrow \widetilde{S}_{g}^{*}
$$

defined by sending the isomorphism class of a curve $C$ to the isomorphism class of the Jacobian variety of $C$. The famous theorem of Torelli asserts that this map $i$ is injective (e.g. [28]). Moreover the map $i$ is holomorphic (and even algebraic). It can be seen by rewriting the map $i$. That is, $\mathcal{S}_{q}^{*}$ is defined analytically as the quotient space of the Siegel upper-half plane $\widetilde{S}_{g}$ of degree $g$ by the integral symplectic group $S p(g, Z)$. It can be considered as the moduli space by letting $\Omega$ mod. $S p(g, Z)$ correspond to the isomorphism class of $C^{g} /\left(1_{g}, \Omega\right) Z^{2 g}$. Then the map $i$ can be defined as the map which sends the isomorphism class of $C$ to the residue class of the period matrix of $C$, and by this definition $i$ is known to be holomorphic (cf. (4.1)).

However the spaces $\mathscr{M}_{g}$ and $\widetilde{S}_{g}^{*}$ are not compact if $g>0$, which gives rise to the problem of their compactification. Several kinds of compactifications with geometrical meaning are known. In case of $\mathscr{M}_{g}$ the moduli space $\mathscr{S}_{g}$ of stable curves of genus $g$ due to Deligne and Mumford gives a good compactification ([4)]. In case of $\mathfrak{S}_{g}^{*}$ the Satake compactification $\overline{\mathfrak{S}}_{g}^{*}$ is a natural one ([19], [20]). As a set $\overline{\mathfrak{S}}_{g}^{*}$ is a union of $\mathfrak{S}_{g^{\prime}}^{*}, 0 \leq g^{\prime} \leq g$. However this compactification has too small boundary (of codimension $g$ ), so $\widetilde{\Xi}_{g}^{*}$ is very singular at the boundary though normal. Igusa studied the desingularization problem

*) This article was presented to Nagoya University for the author's doctorate. 
of the Satake compactification by blowing-up along the boundary ([8]). Unfortunately this procedure does not give the desingularization if $g>3$. The author was informed that now in this direction Mumford and Satake began to study in more general situation (i.e. the desingularization of the Satake compactification of the quotient spaces of bounded symmetric domains). It should be also remarked that the very interesting study on the degeneration of abelian varieties by my colleague, Nakamura ([13]) has a close relation to this problem. It is expected that in the near future we have a nice compactification of $\mathbb{S}_{g}^{*}$ other than Satake compactification. Anyway in this article we shall consider the Satake compactification $\widetilde{\Im}_{g}^{*}$ and the normalization $\widetilde{\Im}_{g}^{*}$ of the blowing-up of $\widetilde{\Im}_{g}^{*}$ along the boundary which we call the Igusa monoidal transform. Denote

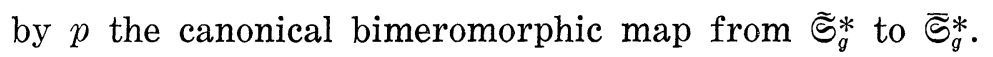

Then the problem arises naturally whether the map $i: \mathscr{M}_{g} \rightarrow \mathfrak{S}_{g}^{*}$ can be extended to a holomorphic map $j: \mathscr{S}_{g} \rightarrow \widetilde{\varsigma}_{g}^{*}$. Our Theorem 4 in $\S 6$ gives the affirmative answer to this problem. The composite map $\bar{j}=$ $p \circ j: \mathscr{S}_{g} \rightarrow \widetilde{\Xi}_{g}^{*}$ sends the isomorphism class of a stable curve $C$ to the isomorphism class of the Jacobian variety of the normalization of $\mathrm{C}$ (Theorem 3 in $\S 5$ ). In fact we show the existence of $\bar{j}$ first and we show that $\bar{j}$ can be lifted to $j$. In the proof we use the methods introduced by Igusa in [8] in its full extent. Especially we use the notions of Fourier-Jacobi series and central cones. For the proof of Theorem 4 we must use the fact that a cone in the vector space of real symmetric matrices generated by a finite number of non-negative integral matrices is covered with a finite number of central cones, which is proved in Theorem 1 in $\S 1$.

After this we shall study the properties of $j$ precisely.

First of all with a stable curve $C$ we associated a (dual) graph whose vertices are the irreducible components of $C$ and whose edges are the double points of $C$ (4.4). We call $C$ planar if the graph associated with $C$ can be embedded in the plane. Those points in $\mathscr{S}_{g}$ corresponding to planar stable curves are mapped by $j$ into "good" points in $\widetilde{\Im}_{g}^{*}$; especially the singularity in $\widetilde{\Im}_{g}^{*}$ of each image point is at most quotient singularity (Theorem 5 in $\S 7$ ).

Secondly denoting by $\mathscr{U}_{g}$ those points in $\mathscr{S}_{g}$ which correspond to irreducible stable curves, we shall study $j$ on $\mathscr{U}_{g}$. Let $C$ be an irreducible stable curve. Then the generalized Jacobian variety $J(C)$ of $C$ is a 
group extension of the Jacobian variety $J(\tilde{C})$ of the normalization $\tilde{C}$ of $C$ by a product of some copies of the multiplicative group $C^{*}$. We note that the extension class of $J(C)$ is explicitly determined by $C$ (Theorem 6 in $\S 8$ ). With the help of this theorem we prove that $j$ is injective on $\mathscr{U}_{g}$ (Theorem 7 ), which gives a natural extension of Torelli's theorem.

Finally we prove that in case of $g=2$ the canonical map $j$ is an isomorphism (Theorem 8 in $\S 9$ ). This fact plays an essential role in the study of degenerated fibres in families of curves of genus two by Ueno and the author ([15]).

This article is divided into 9 sections. The first three sections are preliminary. In Section 1 we recall the notions of fundamental cones and central cones in the theory of positive symmetric matrices due to Igusa [8] and we prove Theorem 1. In Section 2 we recall Satake compactifications and introduce the Igusa monoidal transforms with Igusa's fundamental results in [8]. In Section 3 we make a brief summary on the theory of stable curves due to Deligne and Mumford ([4]).

There is a universal family $\widetilde{\sigma}: \mathscr{Z}_{g} \rightarrow \mathscr{H}_{g}$ of stable curves which is smooth outside a divisor $\mathscr{D}$ in $\mathscr{H}_{g}$ with only normal crossings (3.3). By corresponding $x$ in $\mathscr{H}_{g}-\mathscr{D}$ to the period matrices of $\widetilde{\varpi}^{-1}(x)$ in $\mathfrak{S}_{g}$, we obtain a multiple-valued holomorphic function $T=T_{a}: \mathscr{H}_{g}-\mathscr{D} \rightarrow \mathfrak{S}_{g}$, which is called the period map (of $\widetilde{w}$ ). In Section 4 we introduce this period map and after making a precise study on the homology group of stable curves we study the behaviour of the period map $T$ near the discriminant $\mathscr{D}$ (Proposition 5 and Theorem 2). This result is the foundation of the main theorems in this article.

The rest of this article is devoted to the proof of the main theorems mentioned before.

The author would like to express his hearty thanks to his best friend Dr. Kenji Ueno, whose incessant encouragement and advices were indispensable to this work.

\section{Table of Contents}

List of notations.

$\S 1$. Fundamental cones and central cones.

$\S 2$. Satake compactifications and Igusa monoidal transforms.

$\S 3$. Stable curves and their moduli spaces. 
$\S 4$. Periods of stable curves.

$\S 5$. The canonical map from the moduli space of stable curves to the Satake compactification.

$\S 6$. The canonical map from the moduli space of stable curves to the Igusa monoidal transform.

$\S 7$. The image by $j$ of points corresponding to planar stable curves.

$\S 8$. The case of irreducible stable curves.

$\S 9$. The case of $g=2$.

References.

\section{List of Notations}

$\mathfrak{B}_{g}(n)=\widetilde{\mathfrak{S}}_{g}^{*}(n)-\widetilde{\varsigma}_{g}^{*}(n),(2.2)$.

$C$ : the field of complex numbers.

$C^{*}=C-\{0\}$ : the multiplicative group.

$C_{\sigma}=\bar{C}_{\sigma} \cap \mathfrak{Y}_{g}^{+}((1.4)$ Def. 2).

$\bar{C}_{\sigma}$ : the central cone associated with $\sigma$ ((1.4) Def. 2).

$C_{\circ}=C_{\sigma_{0}}$ : the principal cone (1.7).

$C\left(a_{1}, \cdots, a_{n}\right)$ : the cone generated by $a_{1}, \cdots, a_{n}$ (1.6).

$\mathscr{D}$ : the discriminant of $\widetilde{\sigma}(4.1)$.

$D\left(a_{1}, \cdots, a_{n}\right)$ : the simplex generated by $a_{1}, \cdots, a_{n}(1.6)$.

e( $)=\exp (2 \pi \sqrt{-1}(\quad)),(2.4)$.

$F_{\sigma}=\bar{F}_{\sigma} \cap \mathfrak{Y}_{g}^{+}$, (1.2).

$\bar{F}_{\sigma}$ : the fundamental cone associated with $\sigma$ ((1.2) Def. 1).

$F_{\circ}=F_{\sigma_{0}}$ where $\sigma_{\circ}$ is defined in (1.3.1).

$G L(g, R)$ : the general linear group of degree $g$ with coefficients in $R$. $\mathscr{H}_{g}^{\circ}=\mathscr{H}_{g}-\mathscr{D}$, (4.1).

$H_{\sigma}\left(\tau^{\prime}, \zeta, \tau^{\prime \prime}\right)$ : Fourier-Jacobi series (2.4.2).

$j: \mathscr{S}_{g} \rightarrow \widetilde{\Im}_{g}^{*}$, (6.1).

$\bar{j}=p \circ j: \mathscr{S}_{g} \rightarrow \overline{\mathfrak{S}}_{g}^{*}$, (Introduction).

$J(C)$ : the (generalized) Jabobian variety of $C$ (8.3).

$M(y)$, (1.4).

$\mathscr{M}_{g}$ : the coarse moduli space of non-singular curves of genus $g$ (Introduction, (6.1)).

$p: \widetilde{\Xi}_{g}^{*} \rightarrow \widetilde{\Xi}_{g}^{*}:$ the canonical surjection (Introduction).

$p_{n}: \widetilde{\Im}_{g}^{*}(n) \rightarrow \widetilde{\Im}_{g}^{*}(n),(2.5)$.

$\boldsymbol{R}$ : the field of real numbers.

$\boldsymbol{R}^{+}$: the set of non-negative real numbers. 
$\mathscr{S}_{g}=\mathscr{H}_{g} / P G L(5 g-6):$ the coarse moduli space of stable curves of genus $g$ (3.3).

$\widetilde{S}_{g}:$ the Siegel upper-half plane of degree $g(2.1)$.

$\Im_{g}^{*}=S p(g, Z) \backslash \Im_{g}:$ the coarse moduli space of principally polarized abelian varieties of dimension $g(2.1)$.

$\widetilde{S}_{g}^{*}(n)=\Gamma_{g}(n) \backslash \Im_{g},(2.1)$

$\widetilde{\Im}_{g}^{*}:$ the Satake compactification of $\mathbb{\subseteq}_{g}^{*}(2.2)$.

$\bar{\Im}_{g}^{*}(n)$ : the Satake compactification of $\mathfrak{S}_{g}^{*}(n)$ ((2.2) Def. 4).

$\hat{\subseteq}_{g}^{*}(n)$ : the monoidal transform of $\bar{\subseteq}_{g}^{*}(n)$ along $\mathfrak{B}_{g}(n),(2.5)$.

$\widetilde{\Im}_{g}^{*}$ : the Igusa monoidal transform of $\widetilde{\varsigma}_{g}^{*},(2.6)$.

$\widetilde{\Im}_{g}^{*}(n)$ : the Igusa monoidal transform of $\bar{\Im}_{g}^{*}(n),((2.6)$ Def. 5).

$\widetilde{\Im}_{g}^{\circ}(n)$ : the set of those points in $\widetilde{\Im}_{g}^{*}(n)$ which are conjugate to limits of points in $\widetilde{S}_{g}^{*}(n)$ with normal coordinates bounded above (2.5).

$S p(g, R)$ : the symplectic group of degree $g$ with coefficients in $R(2.1)$. $T=T_{\sigma}: \mathscr{H}_{g}^{\circ} \rightarrow \widetilde{S}_{g}:$ the period map associated with $\widetilde{\sigma}(4.2)$.

$T_{\pi}: S \rightarrow \widetilde{\subseteq}_{g}$ : the period map associated with a family $\pi: X \rightarrow S((4.2)$ Def. 8).

$\operatorname{tr}()$ : the trace function (1.1).

$\mathscr{U}_{g}$ : the open set of points in $\mathscr{S}_{g}$ corresponding to irreducible stable curves (8.0).

$Y_{g}^{+}, Y^{+}$: the set of positive integral matrices (1.1).

$\bar{Y}_{g}^{+}, \bar{Y}^{+}:$the set of non-negative integral matrices (1.1).

$Y_{g}^{*}$ : the set of positive half-integer matrices (1.1).

$Y_{g}^{\circ}$ : the set of positive half-integer matrices $\sigma$ with $C_{\sigma}^{\circ} \neq \phi$ (1.5).

$\mathfrak{Y}_{g}^{+}, \mathfrak{Y}^{+}$: the set of positive real matrices (1.1).

$\overline{\mathfrak{Y}}_{g}^{+}, \overline{\mathfrak{Y}}^{+}:$the set of non-negative real matrices $(1.1)$.

$Z$ : the ring of integers.

$\boldsymbol{Z}^{+}$: the set of positive integers.

$\Gamma_{g}(n)=\operatorname{Ker}(S p(g, \boldsymbol{Z}) \rightarrow S p(g, \boldsymbol{Z} / n \boldsymbol{Z})):$ the principal congruence subgroup (2.1).

$\mu(y),(1.4)$.

$\varpi: \mathscr{Z}_{g} \rightarrow \mathscr{H}_{g}:$ the universal family of tricanonical embedded stable curves (3.3).

$\Phi: \bar{\varsigma}_{g^{\prime}}^{*}(n) \rightarrow \widetilde{\varsigma}_{g}^{*}(n)$ : the Siegel operator (2.2).

$\omega_{X / S}, \omega_{C}:$ the dualizing sheaves (3.2).

$(\alpha, \beta)$ : the intersection number of $\alpha$ and $\beta$ (4.1).

$1_{g}$ : the identity matrix of degree $g$. 


\section{§1. Fundamental cones and central cones.}

(1.1) Let $\bigvee_{g}$ denote the set of real symmetric matrices of degree $g$, which is a vector space of dimension $N=g(g+1) / 2$. Let $\mathfrak{Y}_{g}^{+}$(or simply $\mathfrak{Y}^{+}$if no confusion occurs) denote the set of positive symmetric matrices of degree $g$. Then $\mathfrak{Y}_{g}^{+}$is an open convex cone in $\mathfrak{Y}_{g}$ and its closure $\overline{\mathfrak{Y}}_{g}^{+}$is the set of non-negative symmetric matrices of degree $g$. We write $y>0$ if $y$ is an element of $\mathfrak{y}^{+}$; we write $y \geq 0$ if $y$ is an element of $\overline{\mathfrak{Y}}^{+}$.

On $\overline{\mathfrak{Y}}_{g}$ there is a non-degenerate bilinear form defined by

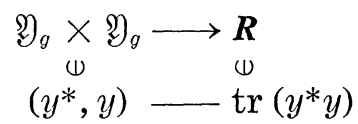

where $\operatorname{tr}(\quad)$ denotes the trace function. With this bilinear form $\mathfrak{V}_{g}^{+}$is the dual cone of itself.

In $\bigvee_{g}$ the set $Y_{g}$ of all integral matrices forms a lattice. Denote $Y_{g} \cap \mathfrak{Y}_{g}^{*}$ by $Y_{g}^{+}$(or simply $Y^{+}$), and $Y_{g} \cap \overline{\mathfrak{Y}}_{g}^{+}$by $\bar{Y}_{g}^{+}$(or simply $\bar{Y}^{+}$) respectively. Note that we can choose a system of generators of $Y_{g}$ in $\bar{Y}_{g}^{+}$. Then the dual lattice $Y_{g}^{* *}$ i.e. the set of matrices with $\operatorname{tr}(\sigma y) \in Z$ for all $y$ in $Y_{g}$ is nothing but the set of half-integer symmetric matrices, i.e. $\sigma=\left(\sigma_{i j}\right)$ with $\sigma_{i i} \in Z$ and $2 \sigma_{i j} \in Z$ for $1 \leq i, j \leq g$. The set $Y_{g}^{*}=$ $Y_{g}^{* *} \cap \bigvee_{g}^{+}$of positive half-integer matrices $\sigma$ is the set of matrices $\sigma$ with $\operatorname{tr}(\sigma y) \in Z^{+}$for all $y$ in $Y_{g}^{+}$.

(1.2) The group $G L(g, R)$ acts continuously on $\mathfrak{Y}_{g}$ as

$$
\begin{aligned}
G L(g, \boldsymbol{R}) \times \mathfrak{Y} & \longrightarrow \mathfrak{W} \\
(u & \mathbb{\Psi} \\
(u, y) & \longrightarrow u \cdot y=u y^{t} u
\end{aligned}
$$

and this action keeps $\mathfrak{Y}^{+}$stable and is transitive on $\mathfrak{Y}^{+}$.

The discontinuous subgroup $G L(g, Z)$ of $G L(g, R)$ acts on $\mathfrak{Y}^{+}$. The reduction theory asserts that the action of $G L(g, Z)$ on $\mathfrak{Y}^{+}$is properly discontinuous and there is a normal fundamental domain with respect to this action.

Following Igusa [8] we shall not consider the fundamental domain itself but a fundamental set $F$ such that $G L(g, Z) \cdot F=\mathfrak{Y}^{+}$and the set $\{u \in G L(g, Z) ; u \cdot F \cap F \neq \phi\}$ is a finite set, or equivalently to say, $F$ is covered with a finite number of fundamental domains.

Definition 1. Choose an element $\sigma$ of $\mathfrak{Y}^{+}$and fix it. Let $\bar{F}_{\sigma}$ be the set of elements $y$ of $\overline{\mathfrak{Y}}$ satisfying 


$$
\operatorname{tr}\left(\sigma u y{ }^{t} u\right)-\operatorname{tr}(\sigma y) \geq 0
$$

for all $u$ in $G L(g, Z)$. We call this $\bar{F}_{\sigma}$ the fundamental cone associated with $\sigma *^{*}$

Also denote $\bar{F}_{\sigma} \cap \mathfrak{Y}^{+}$by $F_{\sigma}$. The $\bar{F}_{\sigma}$ and $F_{\sigma}$ have the following properties :

i) $\bar{F}_{\sigma}$ is a closed convex cone in $\overline{\mathfrak{Y}}^{+}$;

ii) $G L(g, Z) \cdot F_{\sigma}=\mathfrak{Y}^{+}$;

iii) $\left\{u \in G L(g, \boldsymbol{Z}) ; u \cdot F_{\sigma}^{\circ} \cap F_{\sigma} \neq \phi\right\}=\left\{u \in G L(g, \boldsymbol{Z}){ }^{t} u \sigma u=\sigma\right\}$ where $F^{\circ}$ denotes the set of interior points of $F$. We denote the above subgroup of $G L(g, Z)$ by $I(\sigma)$;

iv) ([12]) if moreover $\sigma$ is a half-integer matrix, then for only a finite number of $u$ in $G L(g, Z)$ we have $u \cdot F_{\sigma} \cap F_{\sigma} \neq \phi$. The boundary of $F_{\sigma}$ consists of a finite number of "thin" convex cones. Hence $F_{\sigma}$ is covered with a finite number of fundamental domains.

(1.3) In the following we shall consider a special fundamental cone. Let $\sigma_{\circ}$ be the half-integer matrix

$$
\sigma_{\circ}=\left(\begin{array}{cccc}
1 & 1 / 2 & \cdots & 1 / 2 \\
1 / 2 & 1 & \cdots & 1 / 2 \\
\vdots & \vdots & & \vdots \\
1 / 2 & 1 / 2 & \cdots & 1
\end{array}\right) .
$$

We denote $\bar{F}_{\sigma_{\circ}}\left(\operatorname{resp} . F_{\sigma_{0}}\right)$ simply by $\bar{F}_{\circ}$ (resp. $F_{\circ}$ ).

Denote by $V_{g}$ the real vector space of column vectors with $g$ coefficients. Take a column vector $x$ with coefficients $x_{1}, \cdots, x_{g}$. If we introduce a column vector $\bar{x}$ with coefficients $x_{1}, \cdots, x_{g}, x_{g+1}=-\left(x_{1}+\cdots+x_{g}\right)$, then we have an imbedding $i_{g}: V_{g} \rightarrow V_{g+1}$ whose image is the subspace $V_{g}^{\prime}$ defined by the equation

$$
x_{1}+x_{2}+\cdots+x_{g+1}=0 .
$$

Let $y=\left(y_{i j}\right)$ be a point of $\mathfrak{Y}_{g}$. Introduce a new matrix $\tilde{y}$ in $\mathfrak{Y}_{g+1}$ with coefficients $y_{i j}, 1 \leq i, j \leq g+1$ where additional $g+1$ coefficients are determined by the equations

$$
\sum_{j=1}^{g+1} y_{i j}=0, \quad i=1,2, \cdots, g+1 .
$$

The correspondence $y \rightarrow \tilde{y}$ can be extended to a linear map $\mathfrak{Y}_{g} \rightarrow \mathfrak{V}_{g+1}$.

*) This $\bar{F}_{\sigma}$ was introduced by Venkov [26], Koecher [12] and independently by Igusa [8]. 
With (1.3.2) $\tilde{y}$ is uniquely determined by its $N=g(g+1) / 2$ coefficients $y_{i j}$ for $1 \leq i<j \leq g+1$. It is clear that by these $N$ coefficients $y$ is also determined. Arranging these coordinates lexicographically we call them normal coordinates of $y$. Also we call $\tilde{y}$ the matrix associated with $y$.

Let $\pi_{n}$ be the symmetric group of permutations of the set $\{1, \cdots, n\}$. There is a canonical representation $\pi_{g+1} \rightarrow G L(g+1, Z)$ defined by sending $p=(i \rightarrow p(i))$ to $\bar{u}(p)=\left(\bar{u}(p)_{i j}\right)$ with $\bar{u}(p)_{i j}=1$ if $(i, j)=(i, p(i))$ and 0 otherwise. Clearly this matrix $\bar{u}(p)$ preserves $V_{g}^{\prime}$ above, hence it induces a matrix $u(p)$ in $G L(g, Z)$ through $i_{g}$. Then we have a representation

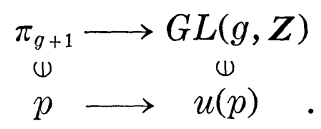

This representation being injective, we identify $\pi_{g+1}$ with its image in $G L(g, Z)$. Then we have

LEMMA $1([8]) . \quad I\left(\sigma_{\circ}\right)=\pi_{g+1} \cup\left(-\pi_{g+1}\right)$.

(1.4) Next we shall introduce another type of closed cone. To define this cone and investigate it we must introduce a few more notations.

Let $y$ be an element of $\overline{\mathfrak{Y}}^{+}=\overline{\mathfrak{Y}}_{g}^{+}$and put

$$
\mu(y)=\inf _{\sigma \in Y_{g}^{*}} \operatorname{tr}(\sigma y),
$$

and

$$
M(y)=\left\{\sigma \in Y_{g}^{*} ; \operatorname{tr}(\sigma y)=\mu(y)\right\} .
$$

LEMMA 2. i) For a positive real number $\lambda$ we have

$$
\mu(\lambda y)=\lambda \mu(y) \quad \text { and } \quad M(\lambda y)=M(y) .
$$

iii) For every element $u$ of $G L(g, Z)$ we have

$$
\mu\left(u y^{t} u\right)=\mu(y) \quad \text { and } \quad M\left(u y{ }^{t} u\right)={ }^{t} u M(y) u .
$$

iii) For $y, y^{\prime} \in \overline{\mathfrak{Y}}$ we have

$$
M(y) \cap M\left(y^{\prime}\right) \subset M\left(\lambda y+\mu y^{\prime}\right)
$$

where $\lambda$ and $\mu$ are positive real numbers.

iv) $\mu(y)$ is upper-semicontinuous. 
The proof is clear.

LEMMA 3. Suppose $y$ is contained in $\mathfrak{Y}^{+}$. Then we have

i) the set of values $\operatorname{tr}(\sigma y)$ with $\sigma \in Y_{g}^{*}$ is a discrete set in $R^{+}$, especially we have $\mu(y)>0$;

ii) $M(y) \neq \phi$ and it is a finite set;

iii) there is a neighbourhood $U$ of $y$ such that for any element of $x$ of $U$ we have $M(x) \subset M(y)$.

Proof. i) It is sufficient to prove that for any number $N$ only a finite number of $\sigma$ 's in $Y_{g}^{*}$ satisfy the inequality

$$
\operatorname{tr}(\sigma y)<N \text {. }
$$

On the other hand for a sufficiently small positive number $\varepsilon$ we have $y \geq \varepsilon 1_{g}$ where $1_{g}$ denotes the identity matrix in $\bigvee_{g}$ (for example, $\varepsilon=$ the least eigenvalue of $y$ ). Then if $\sigma$ satisfies (1.4.1), we have

$$
N>\operatorname{tr}(\sigma y) \geq \operatorname{tr}\left(\sigma\left(\varepsilon 1_{g}\right)\right)=\varepsilon \operatorname{tr} \sigma .
$$

Since $\sigma$ is positive definite, there are only a finite number of such $\sigma$ 's.

ii) This is clear from the proof of $i$ ).

iii) In the same way as in the proof of i) we see that for any compact set $K$ in $\mathfrak{Y}^{+}$and any real number $N$, only a finite number of $\sigma^{\prime}$ 's in $Y^{*}$ satisfy the inequality

$$
\operatorname{tr}(\sigma x)<N
$$

for an $x$ in $K$.

Let $\mu(y)+\varepsilon$ be the smallest value of $\operatorname{tr}(\sigma y)$ but $\mu(y)$. Take a neighbourhood $V$ of $y$ whose closure $\bar{V}$ is compact and contained in $\mathfrak{Y}^{+}$. Then by the remark above for only a finite number of $\sigma_{i}$ 's, $i=1, \cdots, r$, in $Y_{g}^{*}$ there is an element $x$ in $V$ with $\operatorname{tr}\left(\sigma_{i} x\right)<\mu(y)+\varepsilon$. Especially $M(y)$ is contained in $\left\{\sigma_{1}, \cdots, \sigma_{r}\right\}$, so assume for example $M(y)=\left\{\sigma_{1}, \cdots, \sigma_{k}\right\}$. Hence for $j \geq k+1$ we have

$$
\operatorname{tr}\left(\sigma_{j} y\right) \geq \mu(y)+\varepsilon .
$$

By continuity of the function $\operatorname{tr}\left(\sigma_{j} \cdot\right)$ there is a neighbourhood $W$ of $y$ contained in $V$ such that for all $x$ in $W$ and for all $j \geq k+1$,

$$
\operatorname{tr}\left(\sigma_{j} x\right)>\mu(y)+\frac{1}{2} \varepsilon .
$$


By the uppersemicontinuity of $\mu(y)$ also there is a neighbourhood $W^{\prime}$ of $y$ such that for all $x$ in $W^{\prime}$ we have

$$
\mu(x)<\mu(y)+\frac{1}{2} \varepsilon .
$$

Hence if we put $U=W \cap W^{\prime}$, then for any point $x$ in $U$ we have

$$
\operatorname{tr}\left(\sigma_{j} x\right) \geq \mu(y)+\frac{1}{2} \varepsilon>\mu(x)
$$

for $j \geq k+1$ and

$$
\operatorname{tr}(\sigma x) \geq \mu(y)+\varepsilon>\mu(x)
$$

for any $\sigma \neq \sigma_{j}(j=1, \cdots, r)$. That is,

$$
M(x) \subset M(y)
$$

LEMMA 4. Put

$$
\mathfrak{Y}_{g, g^{\prime}}^{+}=\left\{y \in \overline{\mathfrak{Y}}_{g}^{+} ; y=\left(\begin{array}{cc}
0 & 0 \\
0 & y^{\prime}
\end{array}\right) \text { where } y^{\prime} \in \mathfrak{Y}_{g^{\prime}}^{+}\right\}
$$

and

$$
\dot{\mathfrak{Y}}_{g}^{+}=\bigcup_{g^{\prime} \leq g} \mathfrak{Y}_{g, g^{\prime}}^{+}
$$

Then for each point $y=\left(\begin{array}{ll}0 & 0 \\ 0 & y^{\prime}\end{array}\right)$ of $\dot{\mathfrak{y}}_{g}^{+}$we have:

i) the set of values $\operatorname{tr}(\sigma y)$ with $\sigma \in Y_{g}^{+}$is a discrete set in $\boldsymbol{R}^{+}$;

ii) $\mu(y)=\mu\left(y^{\prime}\right)>0$ if $y \neq 0$;

iii) $M(y)=\left\{\sigma=\left(\begin{array}{cc}* & * \\ * & \sigma^{\prime}\end{array}\right) ; \sigma^{\prime} \in M\left(y^{\prime}\right)\right\} \neq \phi$;

iv) there is a neighbourhood $U$ of $y$ such that $M(x) \subset M(y)$ for all $x$ in $U$ with $x \geq y$.

Proof. The claims i), ii) and iii) are clear from the fact that for each $y=\left(\begin{array}{ll}0 & 0 \\ 0 & y^{\prime}\end{array}\right)$ with $y^{\prime} \in Y_{g^{\prime}}^{+}$and $\sigma=\left(\begin{array}{cc}* & * \\ * & \sigma^{\prime}\end{array}\right)$ with $\sigma^{\prime} \in Y_{g^{\prime}}^{*}$ we have

$$
\operatorname{tr}(\sigma y)=\operatorname{tr}\left(\sigma^{\prime} y^{\prime}\right)
$$

iv) Let $\mu(y)+\varepsilon$ be the smallest value of $\operatorname{tr}(\sigma y)$ but $\mu(y)$. Since $\mu(y)$ is uppersemicontinuous, there is a neighbourhood $U$ of $y$ for any element $x$ of which

$$
\mu(x)<\mu(y)+\varepsilon
$$


Hence if moreover $x \geq y$, for any $\sigma \in M(x)$

$$
\begin{aligned}
\operatorname{tr}(\sigma y) & \leq \operatorname{tr}(\sigma y)+\operatorname{tr}(\sigma(x-y)) \\
& =\operatorname{tr}(\sigma x) \\
& =\mu(x) \\
& <\mu(y)+\varepsilon .
\end{aligned}
$$

which implies

$$
\operatorname{tr}(\sigma y)=\mu(y)
$$

That is,

$$
\sigma \in M(y)
$$

q.e.d.

DeFinition 2. Let $\sigma$ be an element of $Y_{g}^{*}$. We call the closed convex cone defined as $\bar{C}_{\sigma}=\left\{y \in \overline{\mathfrak{Y}}^{+} ; \sigma \in M(y)\right\}$ the central cone of $\sigma$. Also denote $\bar{C}_{\sigma} \cap \mathfrak{Y}^{+}$by $C_{\sigma}$.

By Lemma 3 ii) $\mathfrak{Y}^{+}$is covered with central cones. There arises naturally the problem whether the fundamental domain is covered with a finite number of central cones. It is the main object in this section to answer this question affirmatively.

Remark. In general $\mu(y)=0$ and $M(y)=\phi$ for $y$ in the boundary $\overline{\mathfrak{Y}}^{+}-\mathfrak{V}^{+}$. For example you can see easily that for $y=\left(\begin{array}{cc}1 & -\sqrt{2} \\ -\sqrt{2} & 2\end{array}\right)$ it holds that $\mu(y)=0$. It seems to me that if the set of values $\operatorname{tr}(\sigma y)$ is discrete (hence $\mu(y)>0$ and $M(y) \neq \phi$ ), then $y$ is conjugate to a point in $\mathfrak{Y}^{+}$. This subject also seems to have a relation to "rational boundary components" in the sense of Baily and Borel ([2]).

(1.5) First of all we shall note some elementary properties of the central cones.

LEMMA 5. i) Let $Y_{g}^{\circ}$ be the subset of $\sigma^{\prime} s$ with $C_{\sigma}^{\circ} \neq \phi$ where $C_{\sigma}^{\circ}$ denotes the set of interior points of $C_{\sigma}$ in $\mathfrak{Y}^{+}$. Then $\mathfrak{Y}^{+}$is covered with $C_{o}$ 's with $\sigma \in Y_{g}^{\circ}$. We call such $C_{\sigma}$ a non-degenerate central cone.

ii) Let $\sigma$ be an element of $Y_{g}^{\circ}$. For a point $y$ in $C_{\sigma}, M(y)=\{\sigma\}$ if and only if $y \in C_{o}^{\circ}$.

iii) Let $\sigma$ be an element of $Y_{g}^{\circ}$ and put $N_{\sigma}=\left\{\tau \in Y_{g}^{\circ} ; C_{o} \cap C_{\tau} \neq \phi\right\}$. Then we have

$$
C_{\sigma}=\left\{y \in \mathfrak{Y}^{+} ; \operatorname{tr}(\tau y) \geq \operatorname{tr}(\sigma y) \text { for all } \tau \in N_{\sigma}\right\} .
$$


Proof. i) The claim is clear by Baire's theorem.

ii) By Lemma 3 iii) the set $\mathscr{Y}_{\sigma}$ of elements $y$ with $M(y)=\{\sigma\}$ is open, hence $\mathscr{Y}_{\sigma} \subset C_{\sigma}^{\circ}$.

Conversely suppose that $y$ is contained in $C_{\sigma}^{\circ}$. Note that $\mu(x)=$ $\operatorname{tr}(\sigma x)$ on $C_{o}$, which is a linear function. Hence if $\sigma^{\prime}$ satisfies the equality $\mu(y)=\operatorname{tr}\left(\sigma^{\prime} y\right)$, we have $\operatorname{tr}\left(\sigma^{\prime} x\right)=\operatorname{tr}(\sigma x)=\mu(x)$ on a neighbourhood $U$ of $y$, since $\operatorname{tr}\left(\sigma^{\prime} x\right) \geq \operatorname{tr}(\sigma x)$ on $U$. Therefore $\sigma^{\prime}=\sigma$.

iii) It is evident that

$$
C_{\sigma} \subset\left\{y \in \mathfrak{Y}^{+} ; \operatorname{tr}(\tau y) \geq \operatorname{tr}(\sigma y) \text { for all } \tau \in N_{\sigma}\right\} \text {. }
$$

We shall prove the converse by reductio ad absurdum. Let $y$ be an element of $\mathfrak{Y}^{+}$with $\operatorname{tr}(\tau y) \geq \operatorname{tr}(\sigma y)$ for all $\tau \in N_{\sigma}$ which is not contained in $C_{\sigma}$. Take an element $x$ of $C_{\sigma}^{\circ}$. Then we have two inequalities:

$$
\begin{aligned}
& \operatorname{tr}(\sigma y) \leq \operatorname{tr}(\tau y) \\
& \operatorname{tr}(\sigma x)<\operatorname{tr}(\tau x) .
\end{aligned}
$$

Since the segment $\overline{x y}$ is not contained in $C_{\sigma}$ by assumption, on it there is a point $z$ in the boundary of $C_{\sigma}$, hence at least an element $\tau$ of $N_{\sigma}$ is contained in $M(z)$. This implies $\operatorname{tr}(\sigma z)=\operatorname{tr}(\tau z)$, but this is impossible by the above inequalities

q.e.d.

(1.6) Let $a_{1}, \cdots, a_{n}$ be $n$ elements in $\overline{\mathfrak{Y}}_{g}^{+}$. We call $D\left(a_{1}, \cdots, a_{n}\right)=$ $\left\{\sum_{i} \lambda_{i} a_{i} ; \lambda_{i} \in \boldsymbol{R}^{+}\right.$and $\left.\sum \lambda_{i}=1\right\}$ the simplex generated by $a_{1}, \cdots, a_{n}$; we call $C\left(a_{1}, \cdots, a_{n}\right)=\left\{\sum_{i} \lambda_{i} a_{i} ; \lambda_{i} \in \boldsymbol{R}^{+}\right\}=\bigcup_{\lambda \in \boldsymbol{R}^{+}} \lambda D\left(a_{1}, \cdots, a_{n}\right)$ the cone generated by $a_{1}, \cdots, a_{n}$.

If all $a_{i}^{\prime}$ s are in $\bar{Y}_{g}^{+}$, we say $C\left(a_{1}, \cdots, a_{n}\right)$ to be integral.

Our main theorem in $\S 1$ is the following.

THEOREM 1. Let $a_{1}, \cdots, a_{n}$ be $n$ integral non-negative matrices of degree $g$, and let $C\left(a_{1}, \cdots, a_{n}\right)$ be the integral cone generated by them. Then $C\left(a_{1}, \cdots, a_{n}\right)$ is covered with a finite number of non-degenerate central cones.

Clearly we have only to prove that $C\left(a_{1}, \cdots, a_{n}\right) \cap \mathfrak{Y}^{+}$is covered with a finite number of central cones.

The reduction theory asserts that the fundamental domain in $\mathfrak{Y}_{g}^{+}$ with respect to $G L(g, Z)$ is a finite union of integral cones (e.g. cf. [11]). Hence we have: 
COROLlary 1. A finite union of fundamental domains in $\mathfrak{Y}_{g}^{+}$with respect to $G L(g, Z)$ is covered with a finite number of non-degenerate central cones. Especially the fundamental cone associated with a halfinteger matrix is covered with them.

COROLlaRY 2. For a half-integer positive matrix $\sigma$ the central cone $C_{\sigma}$ is a finite union of integral cones and has only a finite number of neighbouring non-degenerate central cones.

Proof. If we prove the finiteness of the number of neighbouring non-degenerate central cones, then the other statements are clear from the definition and Lemma 5 iii). On the other hand let $S$ be the set of non-degenerate central cones which cover the union of $F_{\sigma}$ and its neighbouring fundamental cones. Then $S$ is a finite set by Corollary 1 . Since $C_{\sigma}$ is contained $F_{\sigma}$, every neighbouring central cone of $C_{\sigma}$ belongs to $S$, which proves the assertion.

q.e.d.

To state the next corollary we shall introduce a stratification of $\mathfrak{Y}^{+}$. For a finite subset $M$ in $Y_{g}^{*}$ we define the stratum $\mathscr{Y}_{M}$ associated with $M$ as the set of points $y$ in $\mathfrak{y}^{+}$with $M(y)=M$. By virtue of Lemma 3 ii) and iii) these strata cover $\mathfrak{Y}^{+}$and are locally finite.

It is also easy to see that each stratum is locally an integral cone and on it $\mu(y)$ is a linear function. Hence as the similar way as the proof of Lemma 5 ii) we have

LEMMA 6. If we consider the stratification each stratum of which is the set of points $y$ in $\mathfrak{Y}^{+}$with $M(y) \cap Y_{g}^{\circ}=M^{\circ}$ for a finite subset $M^{\circ}$ in $Y_{g}^{\circ}$, then this stratification coincides with the one defined above. That is, for each point $y$ in $\mathfrak{Y}^{+}$the set $M(y)$ is determined by $M(y) \cap Y_{g}^{\circ}$.

Hence together with Theorem 1 and Corollary 2 we have

COROLlary 3. i) Every integral cone is covered with a finite strata. (Note that every integral cone is contained in the interior of a larger integral cone in $\mathfrak{Y}^{+}$.)

ii) Each stratum is a finite union of integral cones.

iii) If $\overline{\mathscr{Y}}_{M} \supset \mathscr{Y}_{N}$, then $M \subset N$.

iv) $\overline{\mathscr{Y}}_{M}=\bigcap_{\sigma \in M \cap Y^{\circ}} C_{\sigma}$

(1.7) Now let us prove Theorem 1.

First of all we shall reduce the theorem to the case of a special type of cones. 
DEFINITION 3. A cone $C\left(a_{1}, \cdots, a_{n}\right)$ is called regular if

$$
a_{1}>0, \quad a_{1} \geq a_{2} \geq \cdots \geq a_{n} \geq 0 \text {. }
$$

For any cone $C\left(a_{1}, \cdots, a_{n}\right)$ using the barycentric subdivision of $D\left(a_{1}, \cdots, a_{n}\right)$, we have

LEMMA 7 ([11] $\S 4) . \quad$ Put $S=\left\{a_{i_{1}}+\cdots+a_{i_{r}} ; r>0\right\}$. Then $C\left(a_{1}, \cdots\right.$, $\left.a_{n}\right)$ is a union of a finite number of regular cones whose generators are in $S$.

LEMMA 8 ([11] §11). Let $C\left(a_{1}, \cdots, a_{n}\right)$ be a regular integral cone. Then there is a matrix $u$ in $G L(g, Z)$ with $u C\left(a_{1}, \cdots, a_{n}\right)^{t} u \subset \dot{\mathfrak{Y}}_{g}^{+}$, or equivalently, $u a_{i}{ }^{t} u \in \dot{\mathfrak{Y}}_{g}^{+}$for all $i$, where $\dot{\mathfrak{Y}}_{g}^{+}$is defined in (1.4).

Proof. We shall prove this lemma by induction on $g$. In case of $g=1$ there is nothing to prove. Suppose that the claim is true for any $g^{\prime}<g$. Let $k$ be the minimum of $i$ with $\operatorname{det} a_{i}=0$. Then for any $i<k$ we have $a_{i}>0$. Now take an element $u_{1}$ of $G L(g, Z)$ such that $u_{1} a_{k}^{t} u_{1}$ $=\left(\begin{array}{cc}0 & 0 \\ 0 & b_{k}^{\prime}\end{array}\right)$ with $b_{k}^{\prime} \in Y_{g^{\prime}}^{+}\left(g^{\prime}<g\right)$. Since $u_{1} a_{i}{ }^{t} u_{1} \geq u_{1} a_{j}{ }^{t} u_{1}$ for any $k \geq i \geq j$, it follows that $u_{1} a_{i}{ }^{t} u_{1}=\left(\begin{array}{cc}0 & 0 \\ 0 & b_{i}^{\prime}\end{array}\right)$ with $b_{i}^{\prime} \in \bar{Y}_{g}^{+}$, and $b_{k}^{\prime} \geq b_{k+1}^{\prime} \geq \cdots \geq b_{n}^{\prime}$. Hence by assumption there is an element $u_{2}$ of $G L\left(g^{\prime}, Z\right)$ with $u_{2} b_{i}^{\prime} u_{2}=$ $\left(\begin{array}{cc}0 & 0 \\ 0 & b_{i}\end{array}\right), b_{i}>0$. If we put $u=\left(\begin{array}{cc}1_{g-g^{\prime}} & 0 \\ 0 & u_{2}\end{array}\right) u_{1}$, it satisfies the desired condition.

q.e.d.

Secondly we note the following.

Lemma 9. Let $C\left(a_{1}, \cdots, a_{n}\right)$ be a cone in $\dot{\mathfrak{y}}_{g}^{+}$. For any point $y$ in $D\left(a_{1}, \cdots, a_{n}\right)$ there is a neighbourhood $U$ of $y$ with $M(x) \subset M(y)$ for all $x$ in $U \cap D\left(a_{1}, \cdots, a_{n}\right)$.

Proof. We may assume that $a_{1}, \cdots, a_{n}$ are irredundant, i.e. $D\left(a_{1}\right.$, $\left.\cdots, \hat{a}_{i}, \cdots, a_{n}\right) \subseteq D\left(a_{1}, \cdots, a_{n}\right)$ for any $i$ where $D\left(a_{1}, \cdots, \hat{a}_{i}, \cdots, a_{n}\right)$ is the cone generated by $\left\{a_{1}, \cdots, a_{n}\right\}-\left\{a_{i}\right\}$. Assume that

$$
y=\sum \lambda_{i} a_{i}
$$

with $\lambda_{i} \geq 0$. We may further assume that $\lambda_{i}>0$ for $i \leq k$ and $\lambda_{i}=0$ $i>k$. Then we have

$$
D\left(a_{1}, \cdots, a_{n}\right)=\bigcup_{1 \leq j \leq k} D\left(y, a_{1} \cdots, \hat{a}_{i}, \cdots, a_{n}\right) .
$$

Hence we may assume $y=a_{1}$. 
The set $D_{0}=\left\{x=\sum \lambda_{i} a_{i} ; \lambda_{1}>1 / 2\right\}$ is a neighbourhood of $a_{1}$ in $D\left(a_{1}\right.$, $\left.\cdots, a_{n}\right)$. Put $D_{1}=D\left(a_{1}, a_{1}+a_{2}, \cdots, a_{1}+a_{n}\right)$. Then the map

$$
\begin{array}{cc}
p: D_{1} & D_{0} \\
z=\lambda_{1} a_{1}+\sum_{i \geq 2}^{\mathcal{W}} \lambda_{i}\left(a_{1}+a_{i}\right) \longrightarrow z /\left(2-\lambda_{1}\right)
\end{array}
$$

is a homeomorphism. Further we have

$$
M(z)=M(p(z))
$$

and

$$
z \geq a_{1} \quad \text { for all } z \text { in } D_{1} \text {. }
$$

By Lemma $4 \mathrm{iv}$ ) there is a neighbourhood $V$ of $a_{1}$ in $D_{1}$ for any element $z$ of which $M(z) \subset M\left(a_{1}\right)$. Hence $U=p(V)$ is a neighbourhood of $a_{1}$ in $D\left(a_{1}, \cdots, a_{n}\right)$ for any element $x$ of which $M(x) \subset M\left(a_{1}\right)$.

q.e.d.

Proof of theorem 1.

By virtue of Lemmas 7 and 8 we have only to prove the theorem for regular integral cones contained in $\dot{\mathfrak{Y}}_{g}^{+}$. (See also Lemma 2 ii).) Moreover since $M(y)$ is invariant under scalar multiplication (Lemma 2 i)), it is sufficient to prove that the simplex $D\left(a_{1}, \cdots, a_{n}\right)$ is covered with a finite number of nondegenerate central cones.

We shall prove it by induction on $g$. In case of $g=1$ the theorem holds trivially. Hence we suppose that $g>1$ and that the theorem is true for all $g^{\prime}<g$.

Let $C\left(a_{1}, \cdots, a_{n}\right)$ be the regular integral cone considered. Let $k$ be the maximum of $i$ with $\operatorname{det} a_{i}>0$. Then we shall prove the theorem by descending induction on $k$. It is easily seen that we may assume that $n$ is equal to $g(g+1) / 2$ and the generators $a_{1}, \cdots, a_{n}$ are linearly independent (i.e. the cone is non-degenerate and its generators are irredundant).

Suppose that $k=n$. Then $D\left(a_{1}, \cdots, a_{n}\right)$ is compact and contained in $\mathfrak{Y}^{+}$. Hence the claim is true for $k=n$ by virtue of Lemma 3 ii) and iii). Now suppose the claim is true for any $k^{\prime}>k$. By assumption we have

$$
a_{k+1}=\left(\begin{array}{cc}
0 & 0 \\
0 & b_{k+1}
\end{array}\right), \quad b_{k+1} \in \mathfrak{Y}_{g^{\prime}}^{+} \quad\left(g^{\prime}<g\right)
$$

and for $j>k$ 


$$
a_{j}=\left(\begin{array}{ll}
0 & 0 \\
0 & b_{j}
\end{array}\right), \quad b_{j} \in \dot{\mathfrak{Y}}_{g^{\prime}}^{+} \cap \bar{Y}_{g^{\prime}}^{+},
$$

hence

$$
D\left(b_{k+1}, \cdots, b_{n}\right) \subset \dot{\mathfrak{Y}}_{g^{\prime}}^{+} .
$$

By the assumption of induction $D\left(b_{k+1}, \cdots, b_{n}\right)$ is covered with a finite number of non-degenerate central cones. Further by Corollary 3, $D\left(b_{k+1}, \cdots, b_{n}\right) \subset \bigcup_{i=1}^{m} D\left(c_{k+1}^{(i)}, \cdots, c_{n}^{(i)}\right)$ such that for each $D\left(c_{k+1}^{(i)}, \cdots, c_{n}^{(i)}\right)$ an open dense subset of it is contained in a stratum $\mathscr{Y}_{M}^{(i)}$.

By this remark we may assume that an open dense subset of $D\left(b_{k+1}\right.$, $\left.\cdots, b_{n}\right)$ is contained in a stratum $\mathscr{Y}_{M}$ in $\mathfrak{V}_{g^{\prime}}^{+}$.

Put $D_{0}=D\left(a_{k+1}, \cdots, a_{n}\right)$ and $D_{1}=D\left(a_{1}, \cdots, a_{k}\right)$.

Take a point $x$ in $D_{0} \cap \mathscr{Y}_{M}\left(\right.$ We identify $y^{\prime}$ in $\overline{\mathfrak{Y}}_{g^{\prime}}$ with $\left(\begin{array}{ll}0 & 0 \\ 0 & y^{\prime}\end{array}\right)$ in $\left.\overline{\mathfrak{Y}}_{g}^{+} \cdot\right)$ and fix it. By Lemma 9 there is a neighbourhood $U$ of $x$ in $\overline{\mathfrak{Y}}_{g}^{+} \cap D\left(a_{1}\right.$, $\left.\cdots, a_{n}\right)$ such that for any $z$ in $U$ we have $M(z) \subset M(x)$.

Therefore for a sufficiently small positive real number $\lambda$ the simplex $D_{1, x}=(1-\lambda) x+\lambda D_{1}$ is contained in $U$. As it is contained in $\mathfrak{V}^{+}$, it is covered with a finite number of non-degenerate central cones $C_{\sigma_{1}}, \ldots, C_{\sigma_{r}}$. By the assumption and by Lemmas 2 iii) and 4 iii) we have

$$
C_{\sigma_{1}} \cup \cdots \cup C_{\sigma_{r}} \supset D^{\prime}=\bigcup_{0 \leq \mu \leq 1} \mu D_{1, x}+(1-\mu) D_{0} .
$$

On the other hand the intersection of $\overline{\mathfrak{V}}_{g^{\prime}}$ and the closure $D^{\prime \prime}$ of $D\left(a_{1}\right.$, $\left.\cdots, a_{n}\right)-D^{\prime}$ is nothing but the boundary of $D_{0}$. Hence $D^{\prime \prime}$ is covered with a finite number of simplexes with $k^{\prime}>k$, so the claim is true for $D^{\prime \prime}$ by the assumption of induction on $k$.

Hence $D=D^{\prime} \cup D^{\prime \prime}$ is covered with a finite number of central cones. Thus the theorem was proved.

(1.7) We shall close this section with a few remarks and problems. Igusa proved that $C_{\sigma_{\circ}}=F_{\sigma_{\circ}}$ if $g=2$, and 3 . This $C_{\sigma_{\circ}}$ was also introduced by Voronoi ([27]) with the name "principal cone". It can be expressed explicitly as the set of matrices whose normal coordinates (1.3) are all non-positive. We shall denote $C_{\sigma_{0}}$ simply by $C_{0}$. In case of $g=4$ when one uses Igusa's result ([8] Lemma 5) he can prove that

$$
F_{\sigma_{\circ}} \subset C_{\sigma_{\circ}} \cup\left(\bigcup_{1 \leq i<j \leq 5} C_{\sigma_{i j}}\right)
$$


where $\sigma_{i j}$ is defined as

$$
\operatorname{tr}\left(\sigma_{i j} y\right)=\operatorname{tr}\left(\sigma_{\circ} y\right)-y_{i j}
$$

for every $y$ in $\mathfrak{Y}_{4}^{+}$. (Here $y_{i j}$ is the $(i, j)$-component of the normal coordinates of $y$ (1.3).)

Now what is the most interesting is that the non-degenerate central cones seem to coincide with the type I cones due to Voronoi ([27]) and Koecher ([11]). The latter is defined as follows. Let $v$ be an element of $\mathfrak{Y}_{g}^{+}$such that the minimum of values ${ }^{t} \mathfrak{g} v \mathfrak{g}$ for $\mathfrak{g} \in Z^{g}$ is equal to 1 and that $\mathfrak{g}{ }^{t} \mathfrak{g}$ with such integral vectors $\mathfrak{g}$ as ${ }^{t} \mathfrak{g} v \mathfrak{g}=1$ generate non-degenerate cone $C_{v}$ in $\overline{\mathfrak{Y}}_{g}^{+}$. Such $C_{v}$ is called the type I cone associated with $v$. Koecher proved that all coefficients of $v$ are rational numbers ([11] p. 405). If one can show that $v$ is in fact a half-integer matrix, the conjecture above is true. By the observation above the conjecture is true for $g \leq 4$.

\section{§ 2. Satake compactifications and Igusa monoidal transforms}

In this section we make a review on Satake compactifications and their monoidal transforms along the boundary which were introduced by Igusa [8]. For details that we omitted to prove here, we refer the reader to [8].

(2.1) Denote by $\widetilde{\varsigma}_{g}$ the set of symmetric matrices of degree $g$ with complex coefficients whose imaginary parts are positive definite. This $\widetilde{S}_{g}$ is called the Siegel upper-half plane of degree $g$. On it acts the symplectic group $S p(g, Z)$ as $\tau \rightarrow M \cdot \tau=(A \tau+B)(C \tau+D)^{-1}$ for $M=\left(\begin{array}{ll}A & B \\ C & D\end{array}\right)$ in $S p(g, Z)$. Let $\Gamma_{g}(n)$ be the kernel of the natural homomorphism $S p(g, Z) \rightarrow S p(g, Z / n Z)$, which is called the principal congruence subgroup of level $n$. This group $\Gamma_{g}(n)$ acts on $\mathfrak{S}_{g}$ properly discontinuously ([25]). Further if $n$ is greater than 2, the action is free. Hence the quotient space $\mathfrak{S}_{g}^{*}(n)=\Gamma_{g}(n) \backslash \mathfrak{S}_{g}$ admits a canonical structure of a normal analytic space and if $n \geq 3$ it is even non-singular. We write simply $\mathfrak{S}_{g}^{*}$ for $\varsigma_{g}^{*}(1)$.

(2.2) Let $A\left(\Gamma_{g}(n)\right)_{k}$ be the vector space of Siegel modular forms of weight $k$, that is, holomorphic functions $\psi$ on $\widetilde{S}_{g}$ such that $\psi(M \cdot \tau)=$ $\operatorname{det}(C \tau+D)^{k} \psi(\tau)$ for every $M$ in $\Gamma_{g}(n)$. Then the projective variety

$$
\overline{\mathfrak{S}}_{g}^{*}(n)=\operatorname{Proj}\left(\bigoplus_{k \geq 0} A\left(\Gamma_{g}(n)\right)_{k}\right)
$$


contains $\mathfrak{S}_{g}^{*}(n)$ as a Zariski open subset.

DEFINITION 4. The projective algebraic variety $\widetilde{\Im}_{g}^{*}(n)$ is called the Satake compactification of $\mathfrak{S}_{g}^{*}(n)$ (cf. [19], [20]).

The boundary $\mathfrak{B}_{g}(n)=\overline{\mathfrak{S}}_{g}^{*}(n)-\widetilde{S}_{g}^{*}(n)$ is a disjoint union of a finite number of copies of $\mathfrak{S}_{g^{\prime}}^{*}(n)$ with $g^{\prime}<g$. This inclusion is defined by the so-called Siegel operator $\Phi$. Put $g^{\prime \prime}=g-g^{\prime}$ and write an element $\tau$ of $\widetilde{S}_{g}$ as

$$
\tau=\left(\begin{array}{cc}
\tau^{\prime} & \zeta \\
t \zeta & \tau^{\prime \prime}
\end{array}\right)
$$

where $\zeta$ is a $g^{\prime} \times g^{\prime \prime}$ matrix. For every $\psi$ in $A\left(\Gamma_{g}(n)\right)_{k}$ we define $\Phi(\psi)$ as

$$
\Phi(\psi)\left(\tau^{\prime}\right)=\lim _{\operatorname{Im} \tau^{\prime \prime} \rightarrow \infty} \psi(\tau),
$$

and then $\Phi(\psi)$ belongs to $A\left(\Gamma_{g^{\prime}}(n)\right)_{k}$. Hence $\Phi$ gives a homomorphism: $A\left(\Gamma_{g}(n)\right) \rightarrow A\left(\Gamma_{g^{\prime}}(n)\right)$ of graded rings, which is surjective up to a finite number of weights. This homomorphism $\Phi$ defines, therefore, an embedding $\Phi^{*}: \widetilde{\mathfrak{S}}_{g}^{*}(n) \rightarrow \widetilde{\mathfrak{S}}_{g^{\prime}}^{*}(n)$ and the image of $\widetilde{S}_{g^{\prime}}^{*}(n)$ by $\Phi^{*}$ is a locally closed algebraic subset in $\bar{\Im}_{g^{\prime}}^{*}(n)$. On $\bar{\Im}_{g}^{*}(n)$ the group $S p(g, Z / n Z)$ acts and this group transforms the image of $\Im_{g^{\prime}}^{*}(n)$ by $\Phi$ to its conjugates, and these conjugates with $g^{\prime}<g$ forms the boundary $\mathfrak{B}_{g}(n)$. In particular if $n=1, \widetilde{\Im}_{g}^{*}=\bar{\Im}_{g}^{*}(1)$ is a union of $\widetilde{S}_{g^{\prime}}^{*}$ with $g^{\prime} \leq g$.

(2.3) A system of fundamental neighbourhoods of the image $\Phi^{*}\left(t^{\prime}\right)$ of a point $t^{\prime}$ in $\mathfrak{S}_{g^{\prime}}^{*}(n)$ is given as follows.

Fix a fundamental domain $F_{g}(n)$ of $\Gamma_{g}(n)$ in $\widetilde{S}_{g}$ such that for all $g^{\prime}<g$ those elements $\tau^{\prime}$ in $\widetilde{\Im}_{g^{\prime}}$ with $\tau=\left(\begin{array}{cc}\tau^{\prime} & \zeta \\ \iota \zeta & \tau^{\prime \prime}\end{array}\right) \in F_{g}(n)$ form a fundamental domain $F_{g^{\prime}}(n)$ of $\Gamma_{g^{\prime}}(n)$ in $\widetilde{S}_{g^{\prime}}$. (For example take the Siegel fundamental domain.) Take a neighbourhood $U$ of $t^{\prime}$ and a positive number $K$. We define $B^{(k)}(U, K)$ with $g^{\prime} \leq k \leq g$ as

$$
\begin{aligned}
& V^{(k)}(U, K)=\left\{\tau=\left(\begin{array}{cc}
\tau^{\prime} & \zeta \\
t \zeta & \tau^{\prime \prime}
\end{array}\right) \bmod . \Gamma_{k}(n) \in \mathbb{S}_{k}^{*} ;\right. \\
& \left.\tau \in F_{k}(n), \tau^{\prime} \bmod . \Gamma_{g^{\prime}}(n) \in U \text { and } \operatorname{Im} \tau^{\prime \prime}>K 1_{k-g^{\prime}}\right\},
\end{aligned}
$$

and $V(U, K)$ as $\cup_{g^{\prime} \leq k \leq g} \Phi_{k}^{*}\left(V^{(k)}(U, K)\right)$. Then these sets $V(U, K)$ form a system of fundamental neighbourhoods of $\Phi^{*}\left(t^{\prime}\right)$ in $\overline{\mathfrak{S}}_{g}^{*}(n)$ when $U$ runs 
over a system of fundamental neighbourhoods of $t^{\prime}$ in $\mathfrak{S}_{g^{\prime}}^{*}(n)$ and $K$ runs over the positive integers ([19] Th. 1).

(2.4) Take a point $\tau_{\circ}^{\prime}$ in $\mathfrak{S}_{g}$, and consider its image in $\widetilde{S}_{g}^{*}(n)$ by $\Phi^{*}$ which we denote by $t^{\prime}$. Then the analytic local ring $\mathcal{O}_{t^{\prime}}$ of $\widetilde{\Xi}_{g}^{*}(n)$ at $t^{\prime}$ consists of the so-called Fourier-Jacobi series. More precisely Igusa proved the following theorem ([8], Th. 1 and Supplement).

Before the statement of the theorem we shall introduce some preliminary notations. Let $\bar{Y}_{g}^{*}$ be the set of non-negative half-integer matrices and $G L(g, Z)(n)$ be the kernel of the canonical homomorphism: $G L(g, Z) \rightarrow$ $G L(g, Z / n Z)$. Then $G L(g, Z)(n)$ acts on $\bar{Y}_{g}^{*}$ as $\sigma \rightarrow{ }^{t} u \sigma u$ for $u \in G L(g, Z)(n)$. Denote $\exp (2 \pi \sqrt{-1}())$ by $\mathbf{e}()$.

THEOREM. i) The analytic local ring $\mathcal{O}_{t}$, of $\overline{\mathfrak{S}}_{g}^{*}(n)$ at $t^{\prime}$ consists of convergent power series of the form

$$
f\left(\begin{array}{cc}
\tau^{\prime} & \zeta \\
t \zeta & \tau^{\prime \prime}
\end{array}\right)=\sum_{\sigma} H_{\sigma}\left(\tau^{\prime}, \zeta, \tau^{\prime \prime}\right)
$$

where

$$
H_{\sigma}\left(\tau^{\prime}, \zeta, \tau^{\prime \prime}\right)=\sum_{u} \theta_{\sigma}\left(\tau^{\prime}, \zeta^{t} u\right) \mathbf{e}\left((1 / n) \operatorname{tr}\left({ }^{t} u \sigma u \tau^{\prime \prime}\right)\right)
$$

Here the summation in (2.4.1) is taken over a set of representatives of $\bar{Y}_{g^{\prime \prime}}^{*} / G L\left(g^{\prime \prime}, Z\right)(n)$ and the summation in (2.4.2) is taken over all distinct

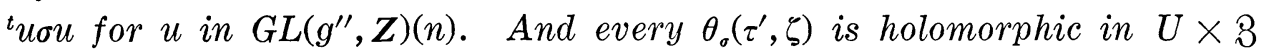
for an open neighbourhood $U$ of $\tau_{\circ}^{\prime}$ in $\widetilde{S}_{g^{\prime}}$ and the vector space 3 of $g^{\prime} \times g^{\prime \prime}$ matrices. Further $\theta_{o}\left(\tau^{\prime}, \zeta\right)$ satisfies the following functional equations :

$$
\begin{aligned}
& \theta_{\sigma}\left(\tau^{\prime}, \zeta+\tau^{\prime} \mathfrak{m}+\mathfrak{n}\right)=\theta_{\sigma}(\tau, \zeta) \mathbf{e}\left(-(1 / n) \operatorname{tr}\left(\sigma\left(2^{t} \mathfrak{m} \zeta+{ }^{t} \mathfrak{m} \tau^{\prime} \mathfrak{n}\right)\right)\right) \\
& \text { where } \mathfrak{m} \text { and } \mathfrak{n} \text { are } g^{\prime} \times g^{\prime \prime} \text { integral matrices } \\
& \theta_{\sigma}\left(M^{\prime} \cdot \tau^{\prime},{ }^{t}\left(C^{\prime} \tau^{\prime}+D^{\prime}\right)^{-1} \zeta\right) \\
& =\theta_{\sigma}\left(\tau^{\prime}, \zeta\right) \mathbf{e}\left((1 / n) \operatorname{tr}\left(\sigma^{t} \zeta\left(C^{\prime} \tau^{\prime}+D^{\prime}\right)^{-1} C^{\prime} \zeta\right)\right) \\
& \text { where } M^{\prime}=\left(\begin{array}{ll}
A^{\prime} & B^{\prime} \\
C^{\prime} & D^{\prime}
\end{array}\right) \in S p\left(g^{\prime}, Z\right)(n) \text { and } M^{\prime} \cdot \tau^{\prime}=\tau^{\prime}
\end{aligned}
$$

ii) The ideal $\mathscr{I}_{t^{\prime}}$ in $\mathcal{O}_{t^{\prime}}$ which defines the boundary $\mathfrak{B}_{g}^{*}(n)$ consists of such series $\sum_{\sigma} H_{\sigma}\left(\tau^{\prime}, \zeta, \tau^{\prime \prime}\right)$ that $\sigma$ is (strictly) positive definite.

(2.5) In [8] Igusa introduced the monoidal transform $\hat{\Im}_{g}^{*}(n)$ of $\widetilde{\Xi}_{g}^{*}(n)$ 
along the boundary $\mathfrak{B}_{g}(n)$ and studied its singularity. We shall use the notations in Section 1 freely.

Denote by $p$ (or $p_{n}$ more precicely) the canonical morphism from $\hat{\Im}_{g}^{*}(n)$ to $\bar{\Im}_{g}^{*}(n)$. Then the singular locus of $\hat{\Im}_{g}^{*}(n)$ is given as follows.

THEOREM ([8] Section 3). Here we suppose that $n \geq 3$.

i) Let $\tilde{t}$ be a point in $\hat{\mathfrak{S}}_{g}^{*}(n)$ and $t=p(\tilde{t})$ in $\overline{\mathfrak{S}}_{g}^{*}(n)$. Then $\tilde{t}$ is a simple point if $\tilde{t}$ is contained in $\mathfrak{S}_{g}^{*}(n)$ or $\tilde{t}$ is a conjugate of a limit of points $\tau^{(k)}=\left(\begin{array}{ll}\tau^{\prime(k)} & \zeta^{(k)} \\ \zeta^{(k)} & \tau^{\prime \prime(k)}\end{array}\right) \bmod . \Gamma_{g}(n)$ such that $\operatorname{Im} \tau^{\prime \prime(k)} \rightarrow \infty$ and the normal coordinates (1.3) of $\operatorname{Im} \tau^{\prime \prime(k)}$ are bounded above, i.e. the distance of $\operatorname{Im} \tau^{\prime \prime(k)}$ and the principal cone $C_{\circ}$ is bounded (cf. (1.7)).

ii) Let $\tilde{t}$ be a limit of points $\tau^{(k)}=\left(\begin{array}{ll}\tau^{\prime(k)} & \zeta^{(k)} \\ t \zeta^{(k)} & \tau^{\prime \prime(k)}\end{array}\right) \bmod . \Gamma_{g}(n)$ with $\operatorname{Im} \tau^{\prime \prime(k)} \rightarrow \infty$ and the normal coordinates of $\operatorname{Im} \tau^{\prime \prime(k)}$ bounded above. Further taking a subsequence if necessary, we may assume that there exist the limits

$$
\begin{aligned}
\tilde{\tau}^{\prime}=\lim _{k \rightarrow \infty} \tau^{\prime(k)}, \tilde{\zeta}=\lim _{k \rightarrow \infty} \zeta^{(k)} \quad \text { and } \quad \tilde{\xi}_{i j}=\lim _{k \rightarrow \infty} \mathbf{e}\left((1 / n)\left(-\tau_{i j}^{\prime \prime(k)}\right)\right), \\
1 \leq i<j \leq g^{\prime \prime}+1,
\end{aligned}
$$

(where $\tau_{i j}^{\prime \prime(k)}$ is the $(i, j)$-component of the normal coordinates of $\left.\tau^{\prime \prime(k)}\right)$. Put $\xi_{i j}=\mathbf{e}\left((1 / n)\left(-\tau_{i j}^{\prime \prime}\right)\right)$. Then a system of local coordinates of $\hat{\mathfrak{S}}_{g}^{*}(n)$ at $\tilde{t}$ is given by

$$
\left(\tau^{\prime}-\tilde{\tau}^{\prime}, \zeta-\tilde{\zeta}, \xi-\tilde{\xi}\right)
$$

iii) The projection of the singular locus of $\hat{\Im}_{g}^{*}(n)$ to $\overline{\mathfrak{S}}_{g}^{*}(n)$ is precisely the union of all conjugates of the image of $\overline{\mathfrak{S}}_{g-4}^{*}(n)$ by the Siegel operator. In particular $\hat{\varsigma}_{g}^{*}(n)$ is non-singular if $g \leq 3$.

We shall denote by $\widetilde{\varsigma}_{g}^{\circ}(n)$ the set of points in $\hat{\subseteq}_{g}^{*}(n)$ satisfying the assumption in the above theorem i).

(2.6) We do not know even whether $\hat{S}_{g}^{*}(n)$ is normal. (It is affirmative if the equality $\left(\mathscr{I}(n)^{r}: \mathscr{I}(n)^{r+s}\right)=\mathscr{I}(n)^{s}$ does hold.) Hence we shall consider the normalization $\widetilde{\Im}_{g}^{*}(n)$ of $\hat{\subseteq}_{g}(n)$.

DEFINITION 5. We call $\widetilde{\Im}_{g}^{*}(n)$ the Igusa monoidal transform of $\bar{\varsigma}_{g}^{*}(n)$ or the Igusa compactification of $\mathfrak{\subseteq}_{g}^{*}(n)$.

In case of $n=1$ we write simply $\widetilde{\Im}_{g}^{*}$ for $\widetilde{\varsigma}_{g}^{*}(1)$.

(2.7) In the following we study the relation between $\widetilde{\Im}_{g}^{*}(n)$ and 
$\widetilde{\Im}_{g}^{*}(m n)$ to show that $\widetilde{\varsigma}_{g}^{*}(n)$ is isomorphic to $(\Gamma(n) / \Gamma(m n)) \backslash \widetilde{\Im}_{g}^{*}(m n)$. We shall begin with a study of Satake compactifications.

We took a note before that the finite group $S p(g, Z / n Z)=S p(g, Z) / \Gamma(n)$ acts on $\widetilde{\subseteq}_{g}^{*}(n)$ complex-analytically (and even algebraically). Further we have:

Proposition 1. i) For each $n, m \geq 1$, there is a canonical morphism $\varphi_{n, m n}: \overline{\mathfrak{S}}_{g}^{*}(m n) \rightarrow \bar{\Im}_{g}^{*}(n)$.

ii) There is an isomorphism $\psi_{n, m n}:(\Gamma(n) / \Gamma(m n)) \backslash \bar{\Im}_{g}^{*}(m n) \rightarrow \bar{\Im}_{g}^{*}(n)$ which satisfies the following commutative diagram;

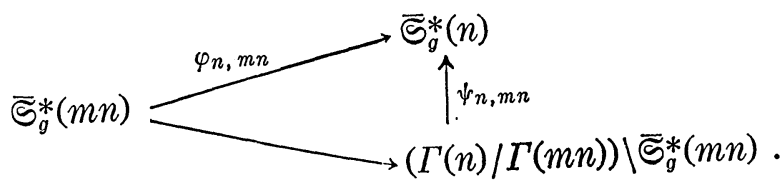

In particular $\varphi_{n, m n}$ is a finite morphism.

Proof. The existence of these morphisms is clear and it is easy to show that $\varphi_{n, m n}$ is a finite morphism. To see $\psi_{n, m n}$ to be isomorphic we have only to use the Zariski main theorem since all varieties are normal.

q.e.d.

Note that the sheaf $\mathscr{I}(m n)$ of ideals of cusp forms on $\widetilde{\Im}_{g}^{*}(m n)$ is stable under the action by $\Gamma(n) / \Gamma(\min )$. Moreover through $\psi_{n, m n}$ we obtain an isomorphism :

$$
\varphi_{n, m n^{*}}(\mathscr{I}(m n))^{\Gamma(n) / \Gamma(m n)} \stackrel{\sim}{\longrightarrow} \mathscr{I}(n) .
$$

Then we have:

Proposition 2. i) For each $m, n \geq 1$, there is a canonical morphism $\tilde{\varphi}_{n, m n}: \widetilde{\Im}_{g}^{*}(m n) \rightarrow \widetilde{\Xi}_{g}^{*}(n)$ which satisfies the following commutative diagram:

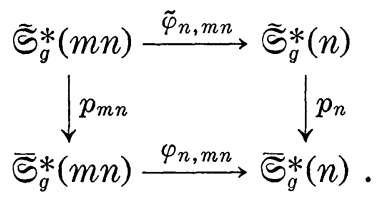

ii) The group $S p(g, Z / n Z)$ acts on $\widetilde{\Im}_{g}^{*}(n)$ and the action is compatible with $p_{n}: \widetilde{\mathfrak{S}}_{g}^{*}(n) \rightarrow \overline{\mathfrak{S}}_{g}^{*}(n)$.

iii) There is an isomorphism $\tilde{\psi}_{n, m n}:(\Gamma(n) / \Gamma(m n)) \backslash \widetilde{\Xi}_{g}^{*}(m n) \rightarrow \widetilde{\Xi}_{g}^{*}(n)$ which satisfies the following commutative diagrams: 

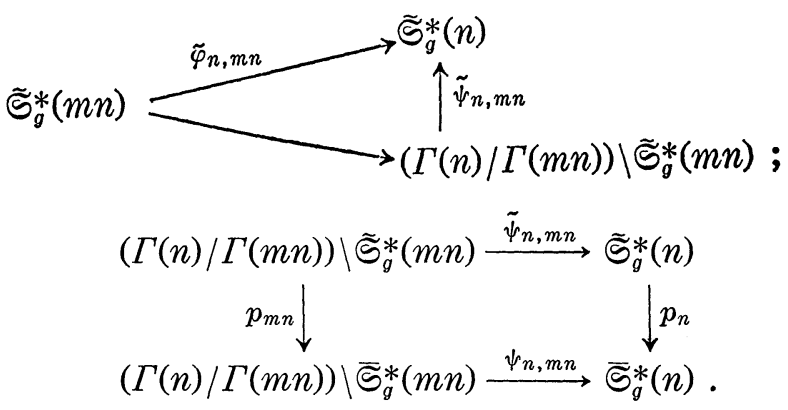

Proof. i) By the remark above we obtain a $\varphi_{n, m n}^{*}$-homomorphism of sheaves of algebras:

$$
\hat{\varphi}_{n, m n}^{*}: \bigoplus_{k \geq 0} \mathscr{I}(n)^{k} \longrightarrow \bigoplus_{k \geq 0} \mathscr{I}(m n)^{k}
$$

where $\varphi_{n, m n}^{*}: \mathcal{O}_{\bar{\Xi}_{g}^{*}(n)} \rightarrow \mathcal{O}_{\bar{\Xi}_{g}^{*}(m n)}$ is induced from $\varphi_{n, m n}$.

This homomorphism induces canonically a rational map

$$
\begin{gathered}
\hat{\varphi}_{n, m n}: \operatorname{Proj}\left(\bigoplus_{k \geq 0} \mathscr{I}(m n)^{k}\right) \longrightarrow \operatorname{Proj}\left(\bigoplus_{k \geq 0} \mathscr{I}(n)^{k}\right) \\
\hat{\Im}_{g}^{*}(m n) \longrightarrow
\end{gathered}
$$

and further $\varphi_{n, m n}$ is a morphism in fact by the remark just before Proposition 2. (We have only to use the fact that the support of $\varphi_{n, m n}^{*}(\mathscr{I}(n))$ is the same as that of $\mathscr{I}(m n)$.) Then it can be lifted canonically to a morphism:

$$
\tilde{\varphi}_{n, m n}: \widetilde{\Im}_{g}^{*}(m n) \longrightarrow \widetilde{\Im}_{g}^{*}(n) .
$$

ii) Clear from the remark above.

iii) Since $\hat{\varphi}_{n, m n}$ is induced from $\varphi_{n, m n}^{*}$-homomorphism (2.7.5), it is an affine map ([6] II. 3.5.1). Moreover clearly it is also proper, hence finite by virtue of Chevalley's theorem (ibid. III. 4.4.2). Therefore $\tilde{\varphi}_{n, m n}$ is also a finite morphism. Since $\widetilde{\Im}_{g}^{*}(n)$ and $\widetilde{\Im}_{g}^{*}(n)$ are birational, the rational function field of $\widetilde{\Im}_{g}^{*}(m n)$ is a Galois extension of that of $\widetilde{\Im}_{g}^{*}(n)$ by Proposition 1. Hence the conclusion follows. q.e.d.

\section{§3. Stable curves and their moduli spaces.}

In this section we recall the definition and fundamental properties of stable curves due to Deligne and Mumford [4]. For the proof not given here we refer the reader to their article above. Although their 
method is algebraic, it is the same in the analytic category by virtue of the representability of the Hilbert functor ([21] Exp. 16).

(3.1) Definition 6. Let $S$ be an analytic space. Let $g \geq 2$. We call a morphism $\pi: X \rightarrow S$ a family of stable curves of genus $g$ over $S$ if it satisfies the following conditions:

i) $\pi$ is a proper and flat epimorphism whose fibres are reduced, connected curves;

ii) for each $s \in S$ the fibre $X_{s}=\pi^{-1}(s)$ has only ordinary double points as singularities;

iii) if $\Gamma$ is a non-singular rational component of $X_{s}$, then $\Gamma$ meets the other components of $X_{s}$ in more than two points;

iv) $\operatorname{dim}_{C} H^{1}\left(X_{s}, \mathcal{O}_{X_{s}}\right)=g$.

If $S$ is one point, $X$ is called a stable curve.

(3.2) Since $\pi$ is flat and its fibres are locally complete intersections, $\pi$ is locally a complete intersection. Hence by the theory of duality of coherent sheaves in the analytic category ([17]), we have the following proposition and from it we infer the following theorem in the same way as in [4].

Proposition 3. Let $\pi: X \rightarrow S$ be a family of stable curves of genus $g$ over $S$. Then there is a canonical invertible sheaf $\omega_{X / S}$ on $X$ such that:

i) for all morphism $f: T \rightarrow S, \omega_{X \times S}$ is canonically isomorphic to $f^{*}\left(\omega_{X / S}\right)$;

ii) if $S$ is one point, let $p: \tilde{X} \rightarrow X$ be the normalization of $X$, $x_{1}, \cdots, x_{n}, y_{1}, \cdots, y_{n}$, the points of $\tilde{X}$ such that the $z_{i}=f\left(x_{i}\right)=f\left(y_{i}\right)$, $1 \leq i \leq n$, are the double points of $X$. Then $\omega_{X / S}$ is the sheaf of meromorphic 1-forms $\eta$ on $\tilde{X}$ regular on $\tilde{X}$ except for simple poles at the $x$ 's and $y$ 's and with $\operatorname{Res}_{x_{i}}(\eta)+\operatorname{Res}_{y_{i}}(\eta)=0$;

iii) if $S$ is one point, and $\mathscr{F}$ is a coherent sheaf on $X$, then

$$
\operatorname{Hom}_{C}\left(H^{1}(X, \mathscr{F}), C\right) \cong \operatorname{Hom}_{\mathscr{O}_{X}}\left(\mathscr{F}, \omega_{X / S}\right) .
$$

If $S$ is one point, we denote $\omega_{X / S}$ simply by $\omega_{X}$. Note that if $X$ is a smooth curve, $\omega_{X}$ is nothing but the usual sheaf of holomorphic one forms on $X$.

COROLlaRY. $\pi_{*} \omega_{X / S}$ is a locally free $\mathcal{O}_{S}$-Module of rank $g$, where $\mathcal{O}_{S}$ 
denotes the sheaf of holomorphic functions on $S$. For each $s \in S$ there are a neighbourhood $U$ of $S$ and $g$ sections $\omega_{1}, \cdots, \omega_{g}$ in $\Gamma\left(\pi^{-1}(U), \omega_{X / S}\right)$ such that for each $t$ in $U$ the restrictions $\left(\omega_{i}\right)_{t}, i=1, \cdots, g$, of $\omega$ 's to the fibre $X_{t}=\pi^{-1}(t)$ form a basis of $\Gamma\left(X_{t}, \omega_{X^{t}}\right)$.

Proof. By the conditions i) and iii) of Proposition 3 we have

$$
\begin{aligned}
H^{0}\left(X_{s},\left.\omega_{X / S}\right|_{X_{s}}\right) & \cong H^{0}\left(X_{s}, \omega_{X_{s}}\right) \cong \operatorname{Hom}_{\mathscr{O}_{S} s}\left(\mathcal{O}_{X_{s}}, \omega_{X_{s}}\right) \\
& \cong \operatorname{Hom}_{C}\left(H^{1}\left(X_{s}, \mathcal{O}_{X_{s}}\right) \cdot C\right) .
\end{aligned}
$$

Hence $\operatorname{dim}_{C} H^{0}\left(X_{s},\left.\omega_{X / S}\right|_{X_{s}}\right)=g$ always. By the theorems of Grauert ([5]) the conclusion follows.

(3.3) To construct the moduli space of stable curves we shall make use of Hilbert moduli space. The starting point is the following theorem.

THEOREM. Let $\pi: X \rightarrow S$ be a family of stable curves of genus $g$. Then $\omega_{X / S}^{\otimes n}$ is relatively very ample if $n \geq 3$ and $\pi_{*}\left(\omega_{X / S}^{\otimes n}\right)$ is a locally free sheaf on $S$ of rank $(2 n-1)(g-1)$.

Taking $n=3$, we can realize any family of stable curves as a family of curves in $\boldsymbol{P}^{5 g-6}$ with Hilbert polynomial:

$$
P_{g}(n)=(2 n-1)(g-1) .
$$

Further there is an analytic subspace

$$
\mathscr{H}_{g} \subset \mathbf{H i l b}_{P 5 g-6}^{P_{g}}
$$

of all tricanonically embedded stable curves where Hilb $_{P^{5 g-6}}^{P_{g}}$ is the Hilbert moduli space in $\boldsymbol{P}^{5 g-6}$ with Hilbert polynomial $\boldsymbol{P}_{g}$. Over $\mathscr{H}_{g}$ there is a family $\varpi: \mathscr{Z}_{g} \rightarrow \mathscr{H}_{g}$ of stable curves of genus $g$ with a tricanonical embedding $\mathscr{Z}_{g} \rightarrow \mathscr{H}_{g} \times \boldsymbol{P}^{5 g-6}$ which has the following universal properties:

(3.3.1) let $\pi: X \rightarrow S$ be a family of stable curves of genus $g$ with the relative projective embedding $i: X \rightarrow \boldsymbol{P}^{5 g-6} \times S$ over $S$ such that the inverse by $i$ of the line bundle determined by the hyperplane of $\boldsymbol{P}^{5 g-6}$ is $\omega_{X / S}^{\otimes 3}$. Then there exists a canonical holomorphic map $f: S \rightarrow \mathscr{H}_{g}$ such that $X$ is isomorphic to $S \times{ }_{\mathscr{F}_{g}} \mathscr{Z}_{g}$ over $S$ and the embeddings into $\boldsymbol{P}^{5 g-6}$ $\times S$ are compatible with $f$. This $f$ is uniquely determined by this property.

Note that by virtue of GAGA [22] $\mathbf{H i l b}_{P_{50-6}}^{P_{g}}$ is the analytic space associated with the algebraic Hilbert scheme. 
On $\mathscr{H}_{g}$ acts the reductive group PGL(5g-6). We see that the quotient space $\mathscr{S}_{g}=\mathscr{H}_{g} /$ PGL(5g-6) exists and it is quasi-projective [9], [16]: In fact it is projective and it is a coarse moduli space of stable curves of genus $g$.

(3.4) On the structure of $\mathscr{H}_{g}$ and $\mathscr{Z}_{g}$, with the approximation theorem of Artin [1], we infer the following theorem after Deligne and Mumford ([4] Th. 1.6).

THEOREM. $\mathscr{H}_{g}$ is smooth and the discriminant of $\varpi$ is reduced with only normal crossings.

More precisely, let $x$ be a point in $\mathscr{H}_{g}$ and $C$ be the stable curve in $\mathscr{Z}_{g}$ lying over $x$ with the double points $z_{1}, \cdots, z_{d}$. Then there are a neighbourhood $U$ of $x$ in $\mathscr{H}_{g}$ which is isomorphic to an open set in $C^{N}$ with local coordinates $\left(t_{1}, \cdots, t_{N}\right)$, and a neighbourhood $V_{i}$ of $z_{i}$ in $\mathscr{Z}_{g}$ which is isomorphic to an analytic subset defined by the equation:

$$
u_{i} v_{i}-t_{i}=0
$$

in an open set in $C^{N+2}$ with local coordinates $\left(u_{i}, v_{i}, t_{1}, \cdots, t_{N}\right)$ such that the structure morphism $\varpi: V_{i} \rightarrow U$ is induced from the projection:

$$
\begin{array}{ccc}
C^{N+2} & \longrightarrow & C_{\Psi}^{N} \\
\left(u_{i}, v_{i}, t_{1}, \cdots, t_{N}\right) & \longrightarrow\left(t_{1}, \cdots, t_{N}\right)
\end{array}
$$

through these isomorphisms.

\section{§ 4. Periods of stable curves.}

In this section we study the period map of the family $\widetilde{\sigma}: \mathscr{Z}_{g} \rightarrow \mathscr{H}_{g}$, especially its behaviour near the points corresponding to singular stable curves. For this purpose we shall study the homology group of stable curves and the monodromy of $\varpi$.

A) Period maps of families of smooth curves.

(4.1) Let $\mathscr{H}_{g}^{\circ}$ be the biggest open subset of $\mathscr{H}_{g}$ over which $\varpi$ is smooth, i.e. the complement of the discriminant $\mathscr{D}$ of $\widetilde{\sigma}$. Denote the inverse image $\widetilde{\sigma}^{-1}\left(\mathscr{H}_{g}^{\circ}\right)$ by $\mathscr{Z}_{g}^{\circ}$. For simplicity we write $\mathscr{H}^{\circ}$ and $\mathscr{Z}^{\circ}$ instead of $\mathscr{H}_{g}^{\circ}$ and $\mathscr{Z}_{g}^{\circ}$ in this section. As we have remarked before in (3.2), the sheaf $\omega_{\mathscr{x}^{\circ} / \mathscr{*}^{\circ}}$ is the sheaf of germs of holomorphic relative one forms on $\mathscr{Z}^{\circ}$.

Then $\widetilde{\varpi}: \mathscr{Z}^{\circ} \rightarrow \mathscr{H}^{\circ}$ is a topological fibre bundle whose fibre is a 
compact surface of genus $g$. Hence we have the following lemma.

DEFINITION 7. Let $C$ be a compact topological surface. A free basis $\left\{\alpha_{1}, \cdots, \alpha_{g}, \beta_{1}, \cdots, \beta_{g}\right\}$ of $H_{1}(C, Z)$ is called a canonical basis if the intersection numbers of the $\alpha$ 's and the $\beta^{\prime}$ s are the following:

$$
\begin{aligned}
& \left(\alpha_{i}, \beta_{j}\right)= \begin{cases}1 & \text { if } i=j \\
0 & \text { if } i \neq j,\end{cases} \\
& \left(\alpha_{i}, \alpha_{j}\right)=\left(\beta_{i}, \beta_{j}\right)=0 \quad \text { for } i, j=1, \ldots, g .
\end{aligned}
$$

LEMMA 10. For each point $x$ in $\mathscr{H}^{\circ}$ there exist a neighbourhood $U$ of $\mathscr{H}^{\circ}$ and $2 g$ cycles $\alpha_{1}, \cdots, \alpha_{g}, \beta_{1}, \cdots, \beta_{g}$ on $\varpi^{-1}(U)$ such that for each $y$ in $U$ the restrictions $\left(\alpha_{i}\right)_{y},\left(\beta_{j}\right)_{y}$ of $\alpha_{i}$ and $\beta_{j}$ to the fibre $C_{y}=$ $\widetilde{\varpi}^{-1}(y)$ form a canonical basis of $H_{1}\left(C_{y}, Z\right)$.

(4.2) Let $\pi: X \rightarrow S$ be a smooth family of non-singular curves of genus $g$ and $\widetilde{\Xi}_{g}$ the Siegel upper-half plane of degree $g$.

For each $s \in S$, choose a neighbourhood $U$ of $s, g$ linearly independent sections $\omega_{1}, \cdots, \omega_{g}$ of $\Gamma\left(\pi^{-1}(U), \omega_{X / S}\right)$, and $2 g$ cycles $\alpha_{1}, \cdots, \alpha_{g}, \beta_{1}, \cdots, \beta_{g}$ whose restrictions $\left(\alpha_{i}\right)_{t},\left(\beta_{j}\right)_{t}$ to $X_{t}=\pi^{-1}(t)$ for every $t$ in $U$ form a canonical basis of $H_{1}\left(X_{t}, Z\right)$. (This is possible by the corollary of Proposition 3 and Lemma 10.)

Then we shall define $T_{\pi}$ on $U$ by

$$
\begin{aligned}
T_{\pi}: U & \longrightarrow \mathbb{S}_{g} \\
& t \longrightarrow\left(\int_{\left(\beta_{k}\right)_{t}}\left(\omega_{\ell}\right)_{t}\right)\left(\int_{\left(\alpha_{i}\right)_{t}}\left(\omega_{j}\right)_{t}\right)^{-1}
\end{aligned}
$$

where $\left(\int_{\left(\alpha_{i}\right)_{t}}\left(\omega_{j}\right)_{t}\right)$ and $\left(\int_{\left(\beta_{k}\right) t}\left(\omega_{\ell}\right)_{t}\right)$ are considered as square matrices of degree $g$.

Since $X_{t}$ is non-singular and the $\left(\omega_{i}\right)_{t}$ 's form a basis of the vector space of holomorphic 1-forms on $X_{t}, T_{\pi}(s)$ belongs to $\widetilde{S}_{g}$ by virtue of Riemann's equality and inequality.

Definition 8. We call this multiple-valued map $T_{\pi}: S \rightarrow \widetilde{S}_{g}$ the period map of the family $\pi$.

The matrix $\Omega_{t}=\left(1_{g}, T_{\pi}(t)\right)$ is usually called the period matrix of $X_{t}$ where $1_{g}$ is the identity matrix of degree $g$. Also the matrix $T_{\pi}(t)$ itself is often called the period matrix of $X_{t}$. In this article we use mainly the latter terminology. 
The multiple-valuedness of $T_{x}$ comes from the freedom of choice of canonical bases. In fact let $(\alpha, \beta)$ and $\left(\alpha^{\prime}, \beta^{\prime}\right)$ be two canonical bases. If we express $\left(\alpha^{\prime}, \beta^{\prime}\right)$ with $(\alpha, \beta)$ in the form:

$$
\begin{aligned}
& \beta_{i}^{\prime}=\sum_{j}\left(A_{i j} \beta_{j}+B_{i j} \alpha_{j}\right), \\
& \alpha_{i}^{\prime}=\sum_{j}\left(C_{i j} \beta_{j}+D_{i j} \alpha_{j}\right),
\end{aligned}
$$

then $M=\left(\begin{array}{ll}A & B \\ C & D\end{array}\right)$ is contained in $S p(g, Z)$ where $A=\left(A_{i j}\right), B=\left(B_{i j}\right), C$ $=\left(C_{i j}\right)$ and $D=\left(D_{i j}\right)$ are matrices of degree $g$, and the value $T^{\prime}$ of $T_{\pi}(t)$ defined with $\left(\alpha^{\prime}, \beta^{\prime}\right)$ is expressed with the value $T$ of $T_{\pi}(t)$ defined with $(\alpha, \beta)$ as

$$
T^{\prime}=M \cdot T=(A T+B)(C T+D)^{-1} .
$$

Hence values of $T_{\pi}$ differ only by the action of $S p(g, Z)$ on $\widetilde{S}_{g}$.

When we are given a family of stable curves $\pi: X \rightarrow S$ which is smooth over an open dense subset $S^{\circ}$, there arises a problem on the behaviour of the period map of $\pi$ near $S-S^{\circ}$. Since the family $\varpi: \mathscr{Z}_{g}$ $\rightarrow \mathscr{H}_{g}$ is universal (3.3), we have only to study the period map $T=T_{\text {ø }}$ of $\varpi: \mathscr{Z}^{\circ} \rightarrow \mathscr{H}^{\circ}$.

B) Homology groups of stable curves.

(4.3) For later use we shall make a precise study on the homology group of stable curves.

Let $C$ be a stable curve with the double points $z_{1}, \cdots, z_{d}$. Denote by $f: \tilde{C} \rightarrow C$ the normalization of $C$ and by $x_{1}, \cdots, x_{d}, y_{1}, \cdots y_{d}$ the points of $\tilde{C}$ with $f\left(x_{i}\right)=f\left(y_{i}\right)=z_{i}, 1 \leq i \leq d$. Let $C_{1}, \cdots, C_{r}$ (resp. $\tilde{C}_{1}, \cdots, \tilde{C}_{r}$ ) be the irreducible components of $C$ (resp. $\tilde{C}$ ) where $C_{j}$ corresponds to $\tilde{C}_{j}$ for each $j$. The genus $g^{\prime}$ of $\tilde{C}$ is the sum of the genus $g_{j}$ of $\tilde{C}_{j}$, $1 \leq j \leq r$.

For each $\tilde{C}_{j}$ choose a canonical basis $\alpha_{\tilde{j}+1}, \cdots, \alpha_{\tilde{j}+g_{j}}, \beta_{\tilde{j}+1}, \cdots, \beta_{\tilde{j}+g_{j}}$ $\left(\tilde{j}=\sum_{k<j} g_{k}\right)$ of the first homology group $H_{1}\left(\tilde{C}_{j}, Z\right)$ of $\tilde{C}_{j}$. Then

$$
H_{1}(\tilde{C}, Z)=\bigoplus_{j=1}^{r} H_{1}\left(\tilde{C}_{j}, Z\right)
$$

and the whole $\alpha$ 's and $\beta$ 's form a canonical basis of $H_{1}(\tilde{C}, Z)$

(4.4) To study $H_{1}(C, Z)$ we shall associate a graph $\Gamma$ with $C$ as 
follows (cf. [4]) :

i) the set of vertices of $\Gamma$ is the set $\Gamma_{v}$ of irreducible components of $C$;

ii) the set of edges of $\Gamma$ is the set $\Gamma_{e}$ of the double points of $C$;

iii) the extremities of an edge $z_{i} \in \Gamma_{e}$ are the irreducible components on which $z_{i}$ lies.

This graph is connected by the condition i) in Definition 6 in (3.1).

Then we have:

LEMMA 11. The first homology group $H_{1}(\Gamma, Z)$ of $\Gamma$ is a free abelian group of rank $d-r+1$. Further we can choose a free basis $\gamma_{1}, \cdots, \gamma_{d-r+1}$ such that

i) if $z_{i} \in \Gamma_{e}$ has only one extremity, then $z_{i}$ is one of $r$ 's (with orientation) and no other $\gamma$ than $z_{i}$ passes through $z_{i}$;

ii) if we assume moreover that the graph $\Gamma$ can be embedded into the sphere $S^{2}$ (or equivalently into the Euclidian plane $\boldsymbol{R}^{2}$ ), then for each $\left.z_{i} \in \Gamma_{e}, a\right)$ there is no $\gamma$ passing through $z_{i}$, or $b$ ) there is only one $\gamma$ passing through $z_{i}$, or $c$ ) there are only two r's passing through $z_{i}$ with opposite directions.

Proof. The first claim is easy to prove.

Let $z_{1}, \cdots, z_{e}$ be the edges of $\Gamma$ with only one extremity and put $\gamma_{i}=z_{i}, i=1, \cdots, e$, with a fixed orientation. Consider the graph $\Gamma_{\text {。 }}$ obtained by deleting these $z$ 's. Then we have easily that

$$
H_{1}(\Gamma, Z)=H_{1}\left(\Gamma_{\circ}, Z\right) \oplus\left(\bigoplus_{i=1}^{e} Z_{\gamma_{i}}\right) \text {. }
$$

Hence if we choose a free basis of $H_{1}\left(\Gamma_{\circ}, Z\right)$, then together with $\gamma_{i}$ above they form a free basis of $H_{1}(\Gamma, Z)$ satisfying the condition i).

Now further we assume that $\Gamma$ can be embedded into $S^{2}$. This is equivalent to assume that $\Gamma_{\circ}$ is embedded into $S^{2}$. Take a point $\infty$ in $S^{2}$ outside $\Gamma_{\circ}$. Then the embedding of $\Gamma_{\circ}$ gives a partition of $S^{2}$ into cells, $\Delta_{1}, \cdots, \Delta_{k}$ and $\Delta_{\infty}$ where $\Delta_{\infty}$ contains the point $\infty$. These cells are naturally oriented by the orientation of $S^{2}$. Let $\gamma_{i}$ be the boundary of $\Delta_{i}, 1 \leq i \leq k$. Then it is easy to see that these $\gamma_{i}$ form a basis of $H_{1}\left(\Gamma_{\circ}, Z\right)$ satisfying the condition ii).

DEFINITION 8. A stable curve is called a planar stable curve if the graph associated with it can be embedded into the sphere $S^{2}$ (or equivalently, in the plane). 
(4.5) Now we embed this graph $\Gamma$ into $C$ so that

i) the image of $C_{i} \in \Gamma_{v}$ is a simple point $c_{i}$ in the irreducible component $C_{i}$;

ii) the image of $z_{i} \in \Gamma_{e}$ with extremities $C_{k}, C_{\ell}$ is a path with extremities $c_{k}, c_{\ell}$ which lies on $C_{k}$ and $C_{\ell}$ and passes through $z_{i}$ once, and through no other $z$ 's.

With this embedding and the normalization $f: \tilde{C} \rightarrow C$, we have a homomorphism:

$$
\varphi: H_{1}(\tilde{C}, Z) \oplus H_{1}(\Gamma, Z) \rightarrow H_{1}(C, Z) .
$$

Proposition 4. The homomorphism $\varphi$ is an isomorphism of groups. Hereafter we identify them through $\varphi$.

Proof. We shall prove the lemma by induction on the number $r$ of the components of $C$. In case of $r=1$ or in case $\Gamma$ is a tree, the proof is clear. In the general case take an irreducible component such that the curve $C^{\prime}$ obtained by deleting this component remains connected. We assume for example that the component $C_{r}$ is a such one.

Denote by $\tilde{C}_{r}$ and $\tilde{C}^{\prime}$ the respective normalizations of $C_{r}$ and $C^{\prime}$. Note that $\tilde{C}$ is a disjoint union of $\tilde{C}_{r}$ and $\tilde{C}^{\prime}$

Denote by $\Gamma_{r}$ and $\Gamma^{\prime}$ the intersections of $C_{r}$ and $C^{\prime}$ with the embeded $\Gamma$ respectively. Then $\Gamma_{r}$ and $\Gamma^{\prime}$ are respectively homotopic to the graph of $C_{r}$ and $C^{\prime}$, and $\Gamma_{r} \cap \Gamma^{\prime}=C_{r} \cap C^{\prime}$ is a finite set of points.

Hence by the theorem of Mayer-Vietorius we obtain the following commutative diagram where the horizontal sequences are exact.

$$
\begin{aligned}
& H_{1}\left(\tilde{C}_{r}\right) \oplus H_{1}\left(\tilde{C}^{\prime}\right) \quad H_{1}(\tilde{C}) \\
& \{0\}=H_{1}\left(\Gamma_{r} \cap \Gamma^{\prime}\right) \rightarrow \underset{ }{\oplus} \rightarrow \oplus \rightarrow H_{0}\left(\Gamma_{r} \cap \Gamma^{\prime}\right) \rightarrow H_{0}\left(\Gamma_{r}\right) \oplus H_{0}\left(\Gamma^{\prime}\right) \\
& H_{1}\left(\Gamma_{r}\right) \oplus H_{1}\left(\Gamma^{\prime}\right) \quad H_{1}(\Gamma) \\
& \underset{\downarrow}{\downarrow} \underset{\downarrow}{\downarrow} \underset{\varphi^{\prime}}{\downarrow} \underset{\downarrow}{\downarrow} \underset{H_{1}}{\downarrow}\left(C_{r} \cap C^{\prime}\right) \rightarrow H_{1}\left(C_{r}\right) \stackrel{\downarrow \psi}{\oplus} H_{1}\left(C^{\prime}\right) \rightarrow \underset{H_{1}}{\downarrow}(C) \rightarrow H_{0}\left(C_{r} \cap C^{\prime}\right) \rightarrow H_{0}\left(C_{r}\right) \oplus H_{0}\left(C^{\prime}\right)
\end{aligned}
$$

Evidently $\psi$ and $\psi^{\prime}$ are isomorphic and $\varphi^{\prime}$ is also by the induction hypothesis. Hence the isomorphy of $\varphi$ follows by virtue of the five lemma.

q.e.d.

C) Monodromy of families of stable curves.

(4.6) In this paragraph $\mathrm{C}$ ) we consider only a family $\pi: X \rightarrow D$ of 
stable curves of genus $g$ over a disc $D=\{t \in C ;|t|<\varepsilon\}$ which is smooth on $D^{\prime}=D-\{0\}$. We shall denote by $X_{t}$ the fibre $\pi^{-1}(t)$ over $t$.

At each double point $z$ of $X_{0}$, there is a neighbourhood $U$ of $z$ which is isomorphic to a closed analytic subset defined by the equation $x y-t^{n}$ $=0$ in an open set in $C^{3}=\{(x, y, t)\}$ containing the origin (cf. (3.4)). Hence replacing $z$ by a series of $(n-1)$ projective lines, we obtain a non-singular model $\tilde{X}$ of $X$. Denote by $\tilde{\pi}$ the canonical map from $\tilde{X}$ to $D$. Note that in the preceding discussion in B) we did not use the condition iii) of Definition 6, hence all results in B) hold also for the curve $\tilde{X}_{0}=\tilde{\pi}^{-1}(0)$.

For the fibre $\tilde{X}_{0}=C$ we shall use the same notations as in (4.3). By the remark above, in particular, we can choose a basis $\left\{\alpha_{1}, \cdots, \alpha_{g^{\prime}}\right.$, $\left.\beta_{1}, \cdots, \beta_{g^{\prime}}, \gamma_{1}, \cdots, \gamma_{g^{\prime \prime}}\right\}$ of $H_{1}(C, Z)$ such that the $\alpha_{\tilde{j}+k}, \beta_{\tilde{j}_{+k}}, 1 \leq k \leq g_{j}$, form a canonical basis of $H_{1}\left(\tilde{C}_{j}, Z\right)$ and the $\gamma_{k}, 1 \leq k \leq g^{\prime \prime}$, form a basis of $H_{1}(\Gamma, Z)$ satisfying the conditions in Lemma 11.

Take a point $t_{\circ}$ in $D^{\prime}$. For simplicity we assume $t_{\circ}=1$. Then we have:

LEMMA 12 a) There are families of cycles $\alpha_{1}(t), \cdots, \alpha_{g}(t), \beta_{1}(t)$, $\cdots, \beta_{g}(t), 0 \leq t \leq 1$, in $H_{1}\left(X_{t}, Z\right)$ such that

i) they vary continuously;

ii) for each $0<t \leq 1$ they form a canonical basis of $H_{1}\left(X_{t}, Z\right)$;

iii) $\alpha_{\tilde{j}+k}(t)\left(\operatorname{resp} . \beta_{\tilde{j}+k}(t)\right), 1 \leq k \leq g_{j}$, tends to $\alpha_{\tilde{j}+k}\left(\right.$ resp. $\left.\beta_{\tilde{j}+k}\right)$ if $t$ tends to 0 ;

iv) $\beta_{g^{\prime}+k}(t), 1 \leq k \leq g^{\prime \prime}$, tends to $\gamma_{k}$ if $t$ tends to 0 ;

v) $\alpha_{g^{\prime}+k}(t), 1 \leq k \leq g^{\prime \prime}$, tends to a cycle homologous to zero if $t$ tends to 0 .

b) For each double point $z_{i}$ in $C=\tilde{X}_{0}$ there is a small open neighbourhood of $z_{i}$ which is homeomorphic to a join of two discs meeting at $z_{i}$. Take the boundary $\delta_{i}$ of one of these discs. Then there is a family of non-zero cycles $\delta_{i}(t), 0<t \leq 1$, (called the vanishing cycles associated with $z_{i}$ ) which tends to $\delta_{i}$ when $t$ tends to 0 .

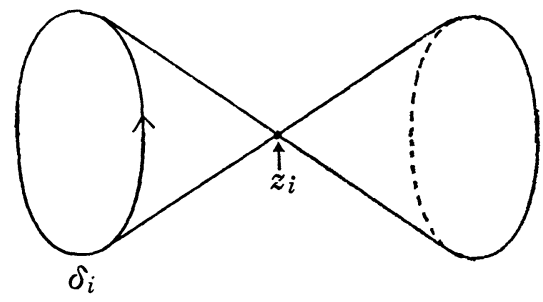


For the proof see [3] Lects. 4 and 5 for example.

As an important corollary of Lemma 11 we have:

LEMMA 13. i) Each $\delta_{i}(t)$ can be expressed as a linear combination of $\alpha_{g^{\prime}+k}(t), 1 \leq k \leq g^{\prime \prime}$.

ii) Further if $C$ is planar (Note that $X_{0}$ is planar if and only if $\tilde{X}_{0}$ is planar.), then $\delta_{i}(t)$ is homologous to zero, or $\pm \alpha_{g^{\prime}+j}(t)$, or $\alpha_{g^{\prime}+j}(t)$ $-\alpha_{g^{\prime}+k}(t)$ with $1 \leq j, k \leq g^{\prime \prime}$.

Proof. i) If we express $\delta_{i}(t)$ in the form

$$
\delta_{i}(t)=\sum_{j=1}^{g} a_{i j} \alpha_{j}(t)+\sum_{j=1}^{g} b_{i j} \beta_{j}(t)
$$

in $H_{1}\left(X_{t}, Z\right)$, then we have

$$
\begin{aligned}
& a_{i j}=\left(\delta_{i}(t), \beta_{j}(t)\right)=\left(\delta_{i}, \beta_{j}\right)=0, \quad 1 \leq j \leq g^{\prime}, \\
& b_{i j}=\left(\delta_{i}(t), \alpha_{j}(t)\right) \begin{cases}=\left(\delta_{i}, \alpha_{j}\right)=0, & 1 \leq j \leq g^{\prime}, \\
=\left(\delta_{i}, 0\right)=0, & g^{\prime}<j \leq g,\end{cases}
\end{aligned}
$$

by Lemma 12 and the condition i) of Lemma 11.

ii) Assume moreover that $C$ is planar. Then by Lemma 11, for each $z_{i}$ in $C$ only one of the following three cases occurs:

a) there is no $\gamma$ passing through $z_{i}$;

b) there is only one $\gamma_{j}$ passing through $z_{i}$;

c) there are two cycles $\gamma_{j}, \gamma_{k}$ passing through $z_{i}$ once with different orientations. Since $a_{i, g^{\prime}+j}=\left(\delta_{i}, \gamma_{j}\right)$, the conclusion follows.

(4.7) Take a circle $\Gamma$ rounding the origin once counterclockwise with base point 1 . For a cycle $c$ in $H_{1}\left(X_{1}, Z\right)$ we denote by $c^{\Gamma}$ the transform of $c$ along $\Gamma$. This operation induces an automorphism of $H_{1}\left(X_{1}, Z\right)$ called the monodromy or the Picard-Lefschetz transformation of $\tilde{\pi}$. Then by the theorem of Picard-Lefschetz we have

$$
c^{\Gamma}=c+\sum_{i=1}^{d}\left(\delta_{i}(1), c\right) \delta_{i}(1)
$$

Let $\mathrm{A}$ be the $d \times g$ matrix with the $(i, j)$-th coefficient $\left(\delta_{i}(1), \beta_{j}(1)\right)$. Then by the above formula we obtain

$$
c^{\Gamma}=c+\alpha(1){ }^{t} A A\left({ }^{t} \alpha(1), c\right) .
$$

Proposition 5. The monodromy of $\pi$ is expressed in the form 


$$
\left(\begin{array}{cc}
1_{g} & B \\
0 & 1_{g}
\end{array}\right), B={ }^{t} A A, 1_{g}=\text { the identity matrix of degree } g
$$

by using the basis $\left\{\beta_{1}(1), \cdots, \beta_{g}(1), \alpha_{1}(1), \cdots, \alpha_{g}(1)\right\}$. Here $B=\left(\begin{array}{ll}0 & 0 \\ 0 & B_{\circ}\end{array}\right)$ where $B_{\circ}$ is a positive definite symmetric matrix of degree $g^{\prime \prime}$.

Moreover if $\tilde{X}_{0}=C$ is planar, then $B_{\circ}$ is contained in the principal cone $C_{\text {。 }}(1.7)$.

Proof. The first part is clear from the argument above except for the claim that $\operatorname{det} B_{\circ} \neq 0$. Since the $\delta_{i}(1)$ 's generate the subgroup generated by $\alpha_{g^{\prime}+1}(1), \cdots, \alpha_{g}(1), \operatorname{rank} A=g^{\prime \prime}$. The formula: $\operatorname{rank}^{t} A A=$ $\operatorname{rank} A$ shows that $\operatorname{det} B_{\circ} \neq 0$.

Denoting by $a_{i}$ the $i$-th row vector of $A$, we have

$$
B=\sum_{i}{ }^{t} \mathfrak{a}_{i} \mathfrak{a}_{i}
$$

If $C$ is planar, then Lemma 13 ii) shows that ${ }^{t} \mathfrak{a}_{i} \mathfrak{a}_{i}=\left(A_{\imath k}^{i}\right)$ is a matrix of one of the following types:

a) $A_{\iota \kappa}^{i}=0,1 \leq \iota, \kappa \leq g$;

b) there is a $j$ with $g^{\prime}<j \leq g$ such that

$$
A_{\iota x}^{i}= \begin{cases}1 & \text { if } \iota=\kappa=j \\ 0 & \text { otherwise }\end{cases}
$$

c) there are $j, k$ with $g^{\prime}<j, k \leq g$ such that

$$
A_{\iota x}^{i}=\left\{\begin{array}{l}
1 \quad \text { if } \iota=\kappa=j \text { or } k \\
-1 \quad \text { if }(\iota, \kappa)=(j, k) \text { or }(k, j) \\
0 \quad \text { otherwise. }
\end{array}\right.
$$

Therefore each minor matrix $A^{i}=\left(A_{\iota x}^{i}\right)_{g^{\prime}<i, \kappa \leq g}$ is contained in $C_{\circ}$, hence $B_{\circ}=\sum A^{i}$ also.

D) Behaviour of the period map near the discriminant.

(4.8) Now we go back to study the period map $T$ of $\varpi: \mathscr{Z}^{\circ} \rightarrow \mathscr{H}^{\circ}$.

Take a point $x$ in $\mathscr{H}_{g}$ and let $C=\widetilde{\sigma}^{-1}(x)$ be the stable curve in $\mathscr{Z}_{g}$ over $x$ with the double points $z_{1}, \cdots z_{d}$. Then by Theorem (3.4) there is a coordinate neighbourhood $U=\left\{\left(t_{1}, \cdots, t_{N}\right) ;\left|t_{i}\right|<\varepsilon\right\}$ with the centre $x$ such that each $z_{i}$ has a neighbourhood biholomorphic to an analytic subset defined by the equation: $x_{i} y_{i}-t_{i}=0$ of an open set in $C^{N+2}=$ 
$\left\{\left(x_{i}, y_{i}, t_{1}, \cdots, t_{N}\right)\right\}$. Hence the discriminant $\mathscr{D}$ is the union $\bigcup_{i=1}^{d}\left\{(t) \in U ; t_{i}\right.$ $=0\}$ in $U$. Its complement $U-\mathscr{D} \cap U$ is isomorphic to $\left(D^{\prime}\right)^{d} \times D^{N-d}$ where $D=\{t \in C ;|t|<\varepsilon\}$ and $D^{\prime}=D-\{0\}$, and the fundamental group of $U-\mathscr{D} \cap U$ is $Z \Gamma_{1} \oplus \cdots \oplus Z \Gamma_{d}$ where $\Gamma_{i}$ is the homotopy class of a circle rounding the divisor $\left\{t_{i}=0\right\}$ once counterclockwise.

Choose a basis of $H_{1}(C, Z)$ as in (4.6). Take a point $x_{1}=\left(t_{1}^{(1)}, \cdots\right.$, $\left.t_{N}^{(1)}\right)$ with $t_{i} \neq 0$ in $U$ sufficiently near to $x$ such that there is a canonical basis of the first homology group $H_{1}\left(C_{1}, Z\right)$ of the non-singular curve $C_{1}=\widetilde{\varpi}^{-1}\left(x_{1}\right)$ such as in Lemma 12 . Then the monodromy of $H_{1}\left(C_{1}, Z\right)$ along $\Gamma_{i}$ is

$$
M_{i}=\left(\begin{array}{cc}
1_{g} & B_{i} \\
0 & 1_{g}
\end{array}\right)
$$

where $B_{i}=\left(b_{i},{ }_{\iota k}\right)$ is a non-negative symmetric integral matrix of rank at most 1 with $b_{i},{ }_{\iota}=0$ for $\iota \leq g^{\prime}$ or $\kappa \leq g^{\prime}$ (Proposition 5). Hence the analytic continuation $T\left(\Gamma_{i} t\right)$ of $T(t)$ along $\Gamma_{i}$ is subject to

$$
T\left(\Gamma_{i} t\right)=M_{i} \cdot T(t)=T(t)+B_{i} .
$$

Hence the matrix-valued function:

$$
S(t)=T(t)-\sum_{i=1}^{d} \frac{\log t_{i}}{2 \pi \sqrt{-1}} B_{i}
$$

is a single-valued function on $U-\mathscr{D} \cap U$. Our main result in this section is :

THEOREM 2. The function $S(t)$ (4.8.2) is bounded on $U-\mathscr{D} \cap U$. Hence it can be extended to a holomorphic function on $U$ by virtue of Riemann's removable singularity theorem.

Proof. Since a holomorphic function defined except on an analytic subset of codimension two can be extended, we have only to prove the case that $C$ has only one double point by virtue of Theorem (3.4).

Even if $C$ has a double point, the monodromy can be trivial. In this case $T(t)$ is already single-valued and is a map into a bounded domain $\mathfrak{S}_{g}$, hence the conclusion follows.

Now we suppose that the monodromy is non-trivial. Then by Proposition 5 we may assume $B=\left(\begin{array}{ll}0 & 0 \\ 0 & n\end{array}\right), n>0$ (in fact $n=1$ ). Let $S_{i j}(t)$ (resp. $T_{i j}(t)$ ) denote the $(i, j)$-coefficient of $S(t)$ (resp. $T(t)$ ). Then by the 
same argument as above, for all $(i, j)$ but $(g, g), S_{i j}(t)=T_{i j}(t)$ is bounded.

So we must prove that $S_{g g}(t)=T_{g g}(t)-\left(n \log t_{1}\right) /(2 \pi \sqrt{-1})$ is holomorphic on $t_{1}=0$ also. This method of proof is due to Mayer ([3]). Since $\operatorname{Im} T_{g g}(t)>0, e(t)=\exp \left(2 \pi \sqrt{-1} T_{g g}(t)\right)$ is bounded on $U$, hence can be extended to a holomorphic function on $U$. As $e(t)$ does not vanish except on $t_{1}=0$, we have $e(t)=t_{1}^{m} e_{0}(t)$ where $e_{0}(0) \neq 0$, so $S^{\prime}(t)=T_{g g}(t)$ - $(m / 2 \pi \sqrt{-1}) \log t_{1}$ is single-valued and holomorphic on a neighbourhood of $x$. If $m \neq n$, then $S^{\prime}(t)$ cannot be single-valued. This shows that $m=n$ and $S^{\prime}(t)=S_{g g}(t)$ is holomorphic at $t_{1}=0 . \quad$ q.e.d.

\section{\$5. The canonical map from the moduli space of stable curves to the Satake compactification.}

In this section we shall prove the following theorem.

THEOREM 3. Let $\pi: X \rightarrow S$ be a family of stable curves of genus $g$. Denote $\operatorname{Sp}(g, Z) \backslash \mathfrak{S}_{g}$ by $\mathfrak{S}_{g}^{*}$. Then there is a canonical holomorphic map

$$
\bar{T}_{\pi}^{*}: S \rightarrow \widetilde{\subseteq}_{g}^{*}=\bigcup_{g^{\prime} \leq g} \widetilde{S}_{g^{\prime}}^{*} \quad \text { (cf. (2.2)) }
$$

sending $s \in S$ to the period matrix of the non-singular model of $X_{s}=\pi^{-1}(s)$.

Proof. By (3.3.1) there is a functorial map $f: S \rightarrow \mathscr{H}_{g}$ such that $X$ is isomorphic to $S \times \mathscr{\mathscr { A }}_{g} \mathscr{Z}_{g}$. Hence we have only to prove the map $\bar{T}_{\sigma_{g}}^{*}: \mathscr{H}_{g} \rightarrow \bar{\Im}_{g}^{*}$ is holomorphic. On the points in $\mathscr{H}_{g}-\mathscr{D}$ whose fibres are smooth curves, this map is nothing but the composition of the canonical surjection $\mathfrak{S}_{g} \rightarrow \mathfrak{S}_{g}^{*}$ and the period map $T$ we have defined in (4.2). Hence it is holomorphic.

The question being local, we consider a point $x \in \mathscr{D} \subset \mathscr{H}_{g}$ and a neighbourhood $U$ of $x$ in Theorem (3.4). Let $C=\varpi^{-1}(x)$ be the stable curve lying over $x$ whose normalization $\tilde{C}$ has a genus $g^{\prime}=g-g^{\prime \prime}$. By virtue of Hartog's theorem we have only to prove that $\bar{T}_{\sigma_{g}}^{*}$ is holomorphic on a neighbourhood of $x$ in a generic line through $x$,i.e., a line which cuts $\mathscr{D}$ transversally.

Denote by $\tau$ a local parameter of a neighbourhood $L$ of $x$ in a line $\left\{\left(\alpha_{1} \tau, \cdots, \alpha_{N} \tau\right) ;|\tau|<\varepsilon\right\}$. By assumption.

$$
T^{*}=\bar{T}_{* \mid L}^{*}: L \rightarrow \bar{\Im}_{g}^{*}
$$

is holomorphic except at $\tau=0$. Hence it is sufficient to show that $T^{*}$ is continuous at $\tau=0$. 
First of all we shall consider the period map $T=T_{\mid L-\{0\}}: L-\{0\} \rightarrow \widetilde{S}_{g}$. By Theorem 2,T( $\tau)$ can be expressed in the form

$$
T(\tau)=\frac{\log \tau}{2 \pi \sqrt{-1}}\left(\begin{array}{cc}
0 & 0 \\
0 & B_{\circ}
\end{array}\right)+S(\tau)
$$

where $B_{\circ}$ is a positive definite symmetric matrix of degree $g^{\prime \prime}$ and $S(\tau)$ is holomorphic on the whole $L$. Let $T^{\prime}$ be the $g^{\prime}$ principal matrix $\left(T_{i j}\right)_{1 \leq i, j \leq g^{\prime}}$ of $T$ which is hence holomorphic on the whole $L$. Then by the definition of the topology of Satake compactification (2.3), we have

$$
\lim _{\tau \rightarrow 0} T^{*}(\tau)=T^{\prime}(0) \bmod S p\left(g^{\prime}, Z\right) .
$$

Hence we have only to prove the following.

Proposition 6. $T^{\prime}(0)$ is the period matrix of the non-singular model $\tilde{C}$ of $C$.

Proof. Let $C_{1}, \cdots, C_{r}$ be the irreducible components of $C$. Then $\tilde{C}$ is a disjoint union of the normalizations $\tilde{C}_{j}$ of $C_{j}, 1 \leq j \leq r$, with genus $g_{j}$. Take such a basis $\left\{\alpha_{1}, \cdots, \alpha_{g^{\prime}}, \beta_{1}, \cdots, \beta_{g^{\prime}}, \gamma_{1}, \cdots, \gamma_{g^{\prime \prime}}\right\}$ of $H_{1}(C, Z)$ as in (4.6).

On the other hand let us consider the dualizing sheaf $\omega_{C}$ on $C$ (3.2). Then we can choose a basis of $\Gamma\left(C, \omega_{C}\right)$ including holomorphic forms $\omega_{\tilde{j}+k}, 1 \leq j \leq r, 1 \leq k \leq g_{j}, \tilde{j}=\sum_{\ell<j} g_{\ell}$, such that for each $j$, the restrictions on $\tilde{C}_{j}$ of $\omega_{\tilde{j}+k}, 1 \leq k \leq g_{j}$, form a basis of $\Gamma\left(\tilde{C}_{j}, \omega_{\widetilde{C}_{j}}\right)$ and they vanish identically on the other $\tilde{C}_{k}^{\text {'s. }}$. Further by a suitable change of basis we may assume that

$$
\int_{\alpha \tilde{j}+i} \omega_{\tilde{j}+k}= \begin{cases}1, & i=k \\ 0, & i \neq k\end{cases}
$$

Since $\widetilde{\sigma}_{*} \omega_{\mathscr{z}_{g / \mathscr{*}_{g}}}$ is a locally free sheaf of rank $g$, we can extend these sections to $\omega_{k}(t), 1 \leq k \leq g$, in $\Gamma\left(\widetilde{\varpi}^{-1}(U), \omega_{\mathscr{x}_{g} / \mathscr{P}_{g}}\right)$ where $U$ is a neighbourhood of $x$ in $\mathscr{H}_{g}$.

By Lemma 12 there is a path $\Gamma$ from $x_{1}$ in $U \cap \mathscr{H}_{g}^{\circ}$ to $x$ such that there is a family of canonical bases of $H_{1}\left(C_{t}, Z\right)$ with $C_{t}=\widetilde{\varpi}^{-1}(t), t \in \Gamma$ satisfying the conditions in Lemma 12. In particular for each $1 \leq i \leq g^{\prime \prime}$, $1 \leq k \leq g^{\prime}$ we have

$$
\lim _{t \rightarrow x} \int_{\alpha_{g^{\prime}+i}(t)} \omega_{k}(t)=\int_{\delta_{i}} \omega_{k}=0
$$


since $\delta_{i}$ is homologous to zero.

Hence we have

$$
T_{i j}(0)=\int_{\beta_{j}} \omega_{j}, 1 \leq i, j \leq g^{\prime}
$$

that is,

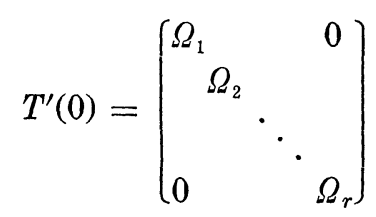

where $\Omega_{j}=\left(\int_{\beta_{\tilde{j}+i}} \omega_{j_{+k}}\right) 1 \leq i, k \leq g_{j}$ is the period matrix of $\tilde{C}_{j}$.

Remark. Since $\mathscr{D}$ is a closed analytic subset in $\mathscr{H}_{g}$ with only normal crossings, the extendability of $T_{\sigma}^{*}: \mathscr{H}_{g}^{\circ} \rightarrow \mathfrak{S}_{g}^{*}$ to $\bar{T}_{\sigma}^{*}: \mathscr{H}_{g} \rightarrow \bar{\Xi}_{g}^{*}$ follows also directly from the theorem of Kobayashi-Ochiai ([10]) in the theory of hyperbolic analysis.

\section{§6. The canonical map from the moduli space of stable curves to the Igusa monoidal transform.}

(6.1) Now we are ready to prove the first main theorem of this article.

THEOREM 4. Let $\pi: X \rightarrow S$ be a family of stable curves of genus $g$. Denote by $\widetilde{\Im}_{g}^{*}$ the Igusa monoidal transform of the Satake compactification $\bar{\Im}_{g}^{*}$ (Definition 5 in (2.6)). Then the canonical map $\bar{T}_{\pi}^{*}: S \rightarrow \widetilde{\Im}_{g}^{*}$ can be lifted to a holomorphic map $\tilde{T}_{\pi}^{*}: S \rightarrow \widetilde{\Xi}_{g}^{*}$.

From this theorem we obtain the following important corollary.

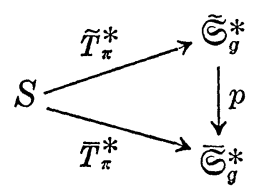

CoRollaRY. Let $\mathscr{M}_{g}$ and $\mathscr{S}_{g}$ be the coarse moduli space of nonsingular curves and stable curves of genus $g$ respectively. Then there is a holomorphic map $j: \mathscr{S}_{g} \rightarrow \widetilde{\Im}_{g}^{*}$ which is an extension of the injection $i: \mathscr{M}_{g} \rightarrow \mathbb{S}_{g}^{*}$. 
Proof. Clear since $\mathscr{S}_{g}=\mathscr{H}_{g} / P G L(5 g-6)$ and $\bar{T}_{\sigma}^{*}$ is $P G L(5 g-6)$ invariant.

(6.2) Proof of Theorem 4. By the same reason as in the proof of Theorem 3, we have only to prove the theorem for the family $\widetilde{\varpi}_{g}: \mathscr{Z}_{g}$ $\rightarrow \mathscr{H}_{g}$.

On $\mathfrak{S}_{g}^{*}$ the morphism $p$ is isomorphic, hence $T_{\sigma}^{*}$ can be defined and holomorphic on $\mathscr{H}_{g}-\mathscr{D}=\mathscr{H}_{g}^{\circ}$.

Since the question is local, we shall consider a point $x$ in $\mathscr{D}$ and take a neighbourhood $U$ satisfying the condition in (3.4). We shall use the same notations in (3.4).

By Theorem 2 the period map $T$ on $U-\mathscr{D} \cap U$ is subject to

$$
T(t)=\sum_{i=1}^{d} \frac{\log t_{j}}{2 \pi \sqrt{-1}} A_{i}+S(t)
$$

where $A_{i}$ is a non-negative matrix of degree $g$ whose $(i, j)$-coefficient is zero if $i \leq g^{\prime}$ or $j \leq g^{\prime}$ and $S(t)$ is a holomorphic function on the whole $U$.

Take a ramified covering $c: V=\left\{\left(s_{1}, \cdots, s_{N}\right)\right\} \rightarrow U$ defined by sending $s_{i}$ to $t_{i}=s_{i}^{n}, 1 \leq i \leq d$, and to $t_{i}=s_{i}, d<i \leq N$. The ramification locus of $c$ is $E=c^{-1}(\mathscr{D})$. Then the composite map $T_{1}=T \circ c$ is subject to

$$
T_{1}(s)=\sum_{i=1}^{d} \frac{\log s_{i}}{2 \pi \sqrt{-1}} n A_{i}+S_{1}(s)
$$

where $S_{1}(s)=S \circ c$ is holomorphic on $V$. Denote $n A_{i}$ by $B_{i}$. Then the composite map:

$$
T_{1}^{*}=p_{n} \circ T_{1}: V-E \longrightarrow \Im_{g}(n) \longrightarrow \Im_{g}^{*}(n)
$$

satisfies the commutative diagram:

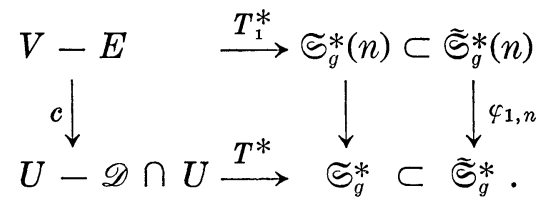

Since these analytic spaces are normal, it suffices to prove that $T_{1}^{*}$ can be extended to a holomorphic map from $V$ to $\widetilde{\varsigma}_{g}^{*}(n)$ for an $n$. Moreover since $\widetilde{\Im}_{g}^{*}(n)$ is the normalization of the monoidal transform $\hat{\Im}_{g}^{*}(n)$, we have only to prove that $T_{1}^{*}$ can be extended to a map from $V$ to $\hat{\varsigma}_{g}^{*}(n)$. 
Let $\bar{T}^{*}: U \rightarrow \bar{\Im}_{g}^{*}$ be the extension of $T^{*}$ in Theorem 3 . Then it is easy to see that there is a holomorphic map $\bar{T}_{1}^{*}: V \rightarrow \bar{\Im}_{g}^{*}(n)$ satisfying the commutative diagram:

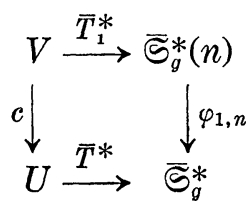

where $\varphi_{1, n}$ is defined in (2.7).

Put $y=\bar{T}_{1}^{*}(x)$, and let $f_{0}, \cdots, f_{s}$ be a system of generators of the ideal $\mathscr{I}_{y}(n)$ of cusp forms at $y$. We may assume that they are holomorphic in a neighbourhood $W$ of $y$. Then the monoidal transform $\hat{\Im}_{g}^{*}(n)$ over $W$ is a strict transform of the image by the map defined by

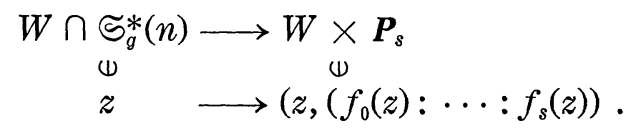

Taking a smaller neighbourhood of $x$ if necessary, we may assume that $\bar{T}_{1}^{*}(V) \subset W$. Hence in order to prove the existence of an extension $\hat{T}_{1}^{*}: V$ $\rightarrow \hat{\Im}_{g}^{*}(n)$ of $T_{1}^{*}$, it is sufficient to prove that the image of $V-E$ by the map $\hat{T}: V-E \rightarrow \boldsymbol{P}_{s}$ defined by sending $z$ to $\left(f_{0}(z): \cdots: f_{s}(z)\right)$ is bounded. In fact we shall prove the following claim:

(*) $\quad V-E$ is covered by a finite union of subsets $V_{i}$ such that for each $i$ there is a unique point $\hat{y}$ in $\boldsymbol{P}_{s}$ with $\lim _{z \rightarrow x, z \in V_{i}} \hat{T}(z)=\hat{y}$.

(6.3) The proof of the claim $(*)$ is done in a few steps.

Write the period map $T_{1}: V-E \rightarrow \mathfrak{S}_{g}$ as

$$
T_{1}(s)=\left(\begin{array}{cc}
T_{1}^{\prime}(s) & T_{1}^{\prime \prime \prime}(s) \\
{ }^{t} T_{1}^{\prime \prime \prime}(s) & T_{1}^{\prime \prime}(s)
\end{array}\right)
$$

where $T_{1}^{\prime \prime \prime}(s)$ is a $g^{\prime} \times g^{\prime \prime}$ matrix, or simply as $\left(T_{1}^{\prime}, T_{1}^{\prime \prime \prime}, T_{1}^{\prime \prime}\right)$.

Then by (6.2.1) $T_{1}^{\prime}(s)$ and $T_{1}^{\prime \prime \prime}(s)$ is holomorphic on the whole $V$ and

$$
T_{1}^{\prime \prime}(s)=\sum_{i=1}^{d} \frac{\log s_{i}}{2 \pi \sqrt{-1}} B_{i}^{\prime \prime}+S_{1}^{\prime \prime}(s)
$$

where $B_{i}^{\prime \prime}$ is non-negative, $B^{\prime \prime}=\sum_{i=1}^{d} B_{i}^{\prime \prime}$ is (strictly) positive and $S_{1}^{\prime \prime}(s)$ is holomorphic on $V$. The imaginary part of $T_{1}^{\prime \prime}(s)$ is hence 


$$
\operatorname{Im} T_{1}^{\prime \prime}(s)=\sum_{i=1}^{d} \frac{-\log \left|s_{i}\right|}{2 \pi} B_{i}^{\prime \prime}+\operatorname{Im} S_{1}^{\prime \prime}(s)
$$

Write also $\tau \in \mathbb{S}_{g}$ as

$$
\tau=\left(\begin{array}{cc}
\tau^{\prime} & \zeta \\
t \zeta & \tau^{\prime \prime}
\end{array}\right)
$$

where $\zeta$ is a $g^{\prime} \times g^{\prime \prime}$ matrix, or simply as $\tau=\left(\tau^{\prime}, \zeta, \tau^{\prime \prime}\right)$. Then $\tau^{\prime}=T_{1}^{\prime}(s)$ (resp. $\zeta=T_{1}^{\prime \prime \prime}(s)$ ) moves a bounded set $K_{1}$ in $\widetilde{S}_{g^{\prime}}$ (resp. $K_{12}$ in the vector space 3 of $g^{\prime} \times g^{\prime \prime}$ matrices) when $s$ moves in $V$.

(6.4) Now we shall use Theorem (2.8) in the full extent. Moreover as you will see soon, our method of proof depends heavily on the method developped by Igusa in [8]. We shall use the same notations as in (2.4).

LEMMA 14 (cf. [8] Lemma 9). Let $\sigma$ be a half-integer positive matrix in $Y^{*}$ and consider a holomorphic function $\theta_{\sigma}\left(\tau^{\prime}, \zeta\right)$ on $K_{1} \times 3$ satisfying the functional equation (2.4.3). Then there are positive constants $\mu, C$ in $\boldsymbol{R}$ such that for $\left(\tau^{\prime}, \zeta\right) \in K_{1} \times K_{12}$ and $u \in G L(g, Z)(n)$ we have

$$
\left|\theta_{\sigma}\left(\tau^{\prime}, \zeta^{t} u\right)\right| \leq C \exp \left(\mu \operatorname{tr}\left({ }^{t} u \sigma u\right)\right) .
$$

Proof. Let $Z$ be the set of points $\tau^{\prime} \mathfrak{m}+\mathfrak{n}$ in 3 where $\tau^{\prime} \in K_{1}$, and $\mathfrak{m}$ and $\mathfrak{n}$ are real $g^{\prime} \times g^{\prime \prime}$ matrices with coefficients in $[0, n]$. Then $Z$ is compact. For every $\zeta \in 3, u \in G L(g, Z)(n)$ and $\tau^{\prime} \in K_{1}$ write $\zeta^{t} u$ in the form $\zeta_{\circ}+\tau^{\prime} \mathfrak{m}+\mathfrak{n}$ with $\zeta_{\circ} \in Z$ and with $\mathfrak{m}, \mathfrak{n} \equiv 0 \bmod . n . \quad$ Put $\operatorname{Re}\left(L_{\tau^{\prime}}(\zeta, \zeta)\right)$ $={ }^{t} \operatorname{Im} \zeta \operatorname{Im}\left(\tau^{\prime}\right)^{-1} \operatorname{Im} \zeta$ after Igusa [8]. Then by (2.4.3) we have

$$
\begin{aligned}
\left|\theta_{\sigma}\left(\tau^{\prime}, \zeta^{t} u\right)\right|= & \left|\theta_{\sigma}\left(\tau^{\prime}, \zeta_{\circ}\right)\right| \exp \left(-(2 \pi / n) \operatorname{tr}\left(\sigma \operatorname{Re}\left(L_{\tau^{\prime}}\left(\zeta_{\circ}, \zeta_{\circ}\right)\right)\right)\right) \\
& \times \exp \left((2 \pi / n) \operatorname{tr}\left(\sigma u\left(\operatorname{Re}\left(L_{\tau^{\prime}}(\zeta, \zeta)\right)\right){ }^{t} u\right)\right) .
\end{aligned}
$$

Since $K_{1}$ and $K_{12}$ are compact, there are positive constants $C$, $\mu$ with

$$
\begin{array}{r}
\left|\theta_{\sigma}\left(\tau^{\prime}, \zeta_{0}\right)\right| \exp \left(-(2 \pi / n) \operatorname{tr}\left(\sigma \operatorname{Re}\left(L_{\tau^{\prime}}\left(\zeta_{\circ}, \zeta_{0}\right)\right)\right)\right)<C \\
\text { for } \tau^{\prime} \in K_{1}, \zeta_{\circ} \in Z
\end{array}
$$

and with $(2 \pi / n) \operatorname{Re}\left(L_{\tau^{\prime}}(\zeta, \zeta)\right)<\mu 1_{g}$ for $\tau^{\prime} \in K_{1}$ and $\zeta \in K_{12}$. Hence we have

$$
\left|\theta_{\sigma}\left(\tau^{\prime}, \zeta^{t} u\right)\right|<C \exp \left(\mu \operatorname{tr}\left({ }^{t} u \sigma u\right)\right)
$$

(6.5) Let $C_{\sigma_{k}}, 1 \leq k \leq p$, be a finite family of non-degenerate central cones whose union $C$ is convex and contains $C\left(B_{1}^{\prime \prime}, \ldots, B_{d}^{\prime \prime}\right)$ and which 
contain the $C_{\sigma}$ 's with $\sigma \in M\left(B^{\prime \prime}\right) \cap Y_{g}^{\circ}$ (cf. (1.4) and (1.5)). We can choose such a family by virtue of Theorem 1 in $\S 1$.

For each $\sigma_{k}$ only a finite number of $u$ in $G L(g, Z)$ satisfy $F_{\sigma_{k}} \cap F_{t_{u \sigma_{k} u}}$ $\neq \phi$. Hence for a sufficiently large $n$ no such an element but the identity is contained in $G L(g, Z)(n)$. That is, $\operatorname{tr}\left(\left({ }^{t} u \sigma u-\sigma\right) y\right)>0$ for $y \in F_{\sigma}$ and $1_{g} \neq u \in G L(g, Z)(n)$. We may also assume $n \geq 3$. We shall fix such an $n$ from now on.

Take an arbitrary positive number $\nu$ and let $\delta$ be a sufficiently large positive number with $\delta B^{\prime \prime} \geq(n / 2 \pi)(\mu+\nu) 1_{g}$ where $\mu$ is the number in Lemma 14.

When $s$ moves in $V, \operatorname{Im} S_{1}^{\prime \prime}(s)$ moves a bounded set $K_{2}$ in the vector space of real matrices of degree $g$. By the assumption above $B^{\prime \prime}$ is contained in the interior of $C$. Hence for a sufficiently large $\varepsilon, K_{2}+\varepsilon B^{\prime \prime}$ is contained in the interior of $C$.

Then we have:

LEMma 15. If $\left|s_{i}\right|<\exp (-2 \pi(\delta+\varepsilon))$, then $\operatorname{Im} T_{1}^{\prime \prime}(s)-\delta B^{\prime \prime} \in C$. Further suppose that $\operatorname{Im} T_{1}^{\prime \prime}(s)-\delta B^{\prime \prime} \in C_{\sigma}$ for $\sigma=\sigma_{k}$. Then the series

$$
\begin{aligned}
& H_{\sigma}\left(T_{1}(s)\right) \mathbf{e}\left(-(1 / n) \operatorname{tr}\left(\sigma T_{1}^{\prime \prime}(s)\right)\right. \\
& \quad=\sum_{u} \theta_{\sigma}\left(T_{1}^{\prime}(s), T_{1}^{\prime \prime \prime}(s) u\right) \mathbf{e}\left((1 / n) \operatorname{tr}\left(\left({ }^{t} u \sigma u-\sigma\right) T_{1}^{\prime \prime}(s)\right)\right)
\end{aligned}
$$

can be dominated by a series

$$
C \sum_{u} \exp \left(-\nu \operatorname{tr}\left({ }^{t} u \sigma u\right)\right)
$$

where $u$ runs over $G L(g, Z)(n)$ and $\nu>0$.

Moreover $H_{\sigma}\left(T_{1}(s)\right) \mathbf{e}\left(-(1 / n) \operatorname{tr}\left(\sigma T_{1}^{\prime \prime}(s)\right)\right)$ converges to $\theta_{\sigma}\left(T_{1}^{\prime}(0), T_{1}^{\prime \prime \prime}(0)\right)$ if $s \rightarrow 0$.

Proof. Put $\tau^{\prime}=T_{1}^{\prime}(s), \zeta=T_{1}^{\prime \prime \prime}(s), \beta=\operatorname{Im} S_{1}^{\prime \prime}(s)$ and $\delta_{i}=-(1 / 2 \pi) \log \left|s_{i}\right|$. Then we have

$$
\begin{aligned}
\operatorname{Im} T_{1}^{\prime \prime}(s)-\delta B^{\prime \prime} & =\sum \delta_{i} B_{i}^{\prime \prime}-\delta B^{\prime \prime}+\beta \\
& =\sum\left(\delta_{i}-\delta-\varepsilon\right) B_{i}^{\prime \prime}+\left(\varepsilon B^{\prime \prime}+\beta\right) \in C .
\end{aligned}
$$

since $\delta_{i}-\delta-\varepsilon>0$ and $\varepsilon B^{\prime \prime}+\beta \in C$.

If we suppose that $\operatorname{Im} T_{1}^{\prime \prime}(s)-\delta B^{\prime \prime} \in C_{\sigma} \subset F_{\sigma}$, then we have

$$
\operatorname{tr}\left(\left({ }^{t} u \sigma u-\sigma\right)\left(\sum \delta_{i} B_{i}^{\prime \prime}-\delta B^{\prime \prime}+\beta\right) \geq 0 .\right.
$$

Hence we have 


$$
\begin{aligned}
& \mid \theta_{\sigma}\left(\tau^{\prime}, \zeta^{t} u\right) \mathbf{e}\left((1 / n) \operatorname{tr}\left(\left({ }^{t} u \sigma u-\sigma\right) T_{1}^{\prime \prime}(s)\right) \mid\right. \\
& \quad=\left|\theta_{\sigma}\left(\tau^{\prime}, \zeta^{t} u\right)\right| \exp \left(-(2 \pi / n) \operatorname{tr}\left(\left({ }^{t} u \sigma u-\sigma\right)\left(\sum \delta_{i} B_{i}^{\prime \prime}+\beta\right)\right)\right) \\
& \quad \leq \text { const. } \exp \left(\mu \operatorname{tr}\left({ }^{t} u \sigma u\right)\right) \exp \left(-(2 \pi / n) \operatorname{tr}\left(\left({ }^{t} u \sigma u-\sigma\right) \delta B^{\prime \prime}\right)\right) \\
& \quad \leq \text { const. } \exp \left(-\nu \operatorname{tr}\left({ }^{t} u \sigma u\right)\right) .
\end{aligned}
$$

Hence the first conclusion follows.

On the other hand with the estimation obtained above we have

$$
\begin{aligned}
\mid \theta_{\sigma}\left(\tau^{\prime}, \zeta^{t} u\right) \mathbf{e}\left((1 / n) \operatorname{tr}\left(\left({ }^{t} u \sigma u-\sigma\right) T_{1}^{\prime \prime}(s)\right) \mid\right. \\
=\left|\theta_{\sigma}\left(\tau^{\prime}, \zeta^{t} u\right)\right| \exp \left(-(2 \pi / n) \operatorname{tr}\left(\left({ }^{t} u \sigma u-\sigma\right)\left(\sum \delta_{i} B_{i}^{\prime \prime}+\beta\right)\right)\right. \\
\leq \text { const. } \exp \left(\mu \operatorname{tr}\left({ }^{t} u \sigma u\right)\right) \exp \left(-(2 \pi / n) \operatorname{tr}\left(\left({ }^{t} u \sigma u-\sigma\right)\left(\sum \sigma_{i} B_{i}^{\prime \prime}+\beta\right)\right)\right. \\
\leq \text { const. } \exp \left(-\nu \operatorname{tr}\left({ }^{t} u \sigma u\right)\right) \exp (-(2 \pi / n) \\
\quad \times \operatorname{tr}\left(\left({ }^{t} u \sigma u-\sigma\right)\left(\sum \delta_{i} B_{i}^{\prime \prime}+\beta-\delta B^{\prime \prime}\right)\right) .
\end{aligned}
$$

By the condition on $n$ above this tends to zero for $s \rightarrow 0$ unless $u$ $=1_{g^{\prime \prime}}$. Together with the first claim the conclusion follows. q.e.d.

LEMMA 16. The assumption is the same as Lemma 15. For any half-integer positive-definite matrix $\sigma^{\prime}$ the series

$$
H_{\sigma^{\prime}}\left(T_{1}(s)\right) \mathbf{e}\left(-(1 / n) \operatorname{tr}\left(\sigma T_{1}^{\prime \prime}(s)\right)\right)
$$

is dominated by a series

$$
C \sum_{u} \exp \left(-\nu^{\prime} \operatorname{tr}\left({ }^{t} u \sigma^{\prime} u\right)\right)
$$

The proof is similar to that of the first part of Lemma 15.

(6.6) Now we shall finish the proof of Theorem 4.

Shrinking $U$ if necessary, we may assume that $\left|s_{i}\right|<\exp (-2 \pi(\delta+\varepsilon))$ on $V$. Put

$$
V_{i}=\left\{s \in V^{\prime} ; \operatorname{Im} T_{1}^{\prime \prime}(s)-\delta B^{\prime \prime} \in C_{\sigma_{i}}\right\} .
$$

Then by assumption $V=\bigcup V_{i}$ and on $V_{i}$ the estimates in Lemmas 15 and 16 hold.

Since $n \geq 3$, by the theorem in the theory of the theta function, for a function $\theta_{\sigma_{i}}^{\circ}\left(\tau^{\prime}, \zeta\right)$ defined near $\left(T_{1}^{\prime}(0), T_{1}^{\prime \prime \prime}(0)\right)$ we have $\theta_{\sigma_{i}}^{\circ}\left(T_{1}^{\prime}(0), T_{1}^{\prime \prime \prime}(0)\right) \neq 0$. Let $H_{\sigma_{i}}^{\circ}(\tau)$ be the Fourier-Jacobi series with the above $\theta_{\sigma_{i}}^{\circ}$. Then by Lemmas 15 and 16 for every $H_{\sigma^{\prime}}(\tau)$ the function

$$
\begin{aligned}
H_{\sigma^{\prime}}\left(T_{1}(s)\right) / H_{\sigma_{i}}\left(T_{1}(s)\right)= & H_{\sigma^{\prime}}\left(T_{1}(s)\right) \mathbf{e}\left(-(1 / n) \operatorname{tr}\left(\sigma_{s} T_{1}^{\prime \prime}(s)\right)\right) \\
& \times\left(H_{\sigma_{i}}^{\circ}\left(T_{1}(s)\right) \mathbf{e}\left(-(1 / n) \operatorname{tr}\left(\sigma_{i} T_{1}^{\prime \prime}(s)\right)\right)\right)^{-1}
\end{aligned}
$$


converges if $s \rightarrow 0$. This proves the claim $(*)$ in (6.2) together with Theorem (2.4), hence the proof of Theorem 4 is complete.

\section{$\S 7$. The image by $\boldsymbol{j}$ of points corresponding to planar stable-curves.}

(7.1) Let $j: \mathscr{S}_{g} \rightarrow \widetilde{\Xi}_{g}^{*}$ be the canonical extension of $i: \mathscr{M}_{g} \rightarrow \widetilde{\subseteq}_{g}^{*}$ obtained in Corollary of Theorem 4.

Recall the set $\widetilde{\Xi}_{g}^{\circ}$ of points which are limits of points on $\widetilde{\varsigma}_{g}^{*}$ with representatives in $\widetilde{S}_{g}$ whose imaginary parts of normal coordinates are bounded above (2.5). We have considered it as a subset of $\hat{\subseteq}_{g}^{*}$ but we can also consider it as a subset of $\widetilde{\Im}_{g}^{*}$ since $\widetilde{\Im}_{g}^{\circ}$ is normal by virtue of Theorem (2.5) and Proposition 2 in (2.7).

Let $x$ be a point in $\mathscr{H}_{g}$ corresponding to a planar stable curve with virtual genus $g^{\prime}=g-g^{\prime \prime}$. Then by virtue of Proposition 5 in (4.7) the period map $T$ near $x$ is subject to

$$
T(t)=\sum_{i=1}^{d} \frac{\log t_{i}}{2 \pi \sqrt{-1}}\left(\begin{array}{ll}
0 & 0 \\
0 & B_{i}^{\prime \prime}
\end{array}\right)+S(t)
$$

where $B_{i}^{\prime \prime}$ is contained in the principal cone $C_{\circ}$, i.e. the normal coordinates of $B_{i}^{\prime \prime}$ are non-positive, and $S(t)$ is holomorphic on a neighbourhood of $x$. Hence the normal coordinates of $\operatorname{Im} T^{\prime \prime}(t)$ are bounded above near $x$ where

$$
T(t)=\left(\begin{array}{cc}
T^{\prime}(t) & T^{\prime \prime \prime}(t) \\
{ }^{t} T^{\prime \prime \prime}(t) & T^{\prime \prime}(t)
\end{array}\right)
$$

with $T^{\prime \prime}(t) \in \subseteq_{g^{\prime \prime}}$. Together with Theorem (2.5) and Proposition 2 we have obtained the following theorem.

THEOREM 5. Every point in $\mathscr{S}_{g}$ corresponding to a planar stable curve is mapped into $\widetilde{\varsigma}_{g}^{\circ}$ by $j$. In particular the image point has at most quotient singularity.

In this case exist limits

$$
\begin{aligned}
\tau^{\prime} & =\lim _{t \rightarrow 0} T^{\prime}(t)=T^{\prime}(x), \\
\zeta & =\lim _{t \rightarrow 0} T^{\prime \prime \prime}(t)=T^{\prime \prime \prime}(x),
\end{aligned}
$$

and

$$
\xi_{i j}=\lim _{t \rightarrow 0} \mathbf{e}\left(-T^{\prime \prime}(t)_{i j}\right)= \begin{cases}0 & \text { if } B_{k, i j}^{\prime \prime} \neq 0 \text { for a } k \\ \mathbf{e}\left(-T^{\prime \prime}(x)_{i j}\right) & \text { otherwise }\end{cases}
$$


where $T^{\prime \prime}(t)_{i j}$ (resp. $B_{k, i j}^{\prime \prime}$ ) denotes the $(i, j)$ coefficient of the normal coordinates of $T^{\prime \prime}(t)$ (resp. $B_{k}^{\prime \prime}$ ). We call $\left(\tau^{\prime}, \zeta, \xi\right)$ the generalized period matrix of the stable curve corresponding to $x$ (cf. (8.2) and (8.4)).

(7.2) Remark. The simplest example of non-planar stable curve is a union of 6 non-singular rational curves with genus 4 such as Fig. 1.

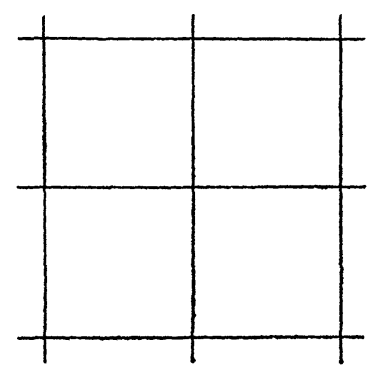

Fig. 1

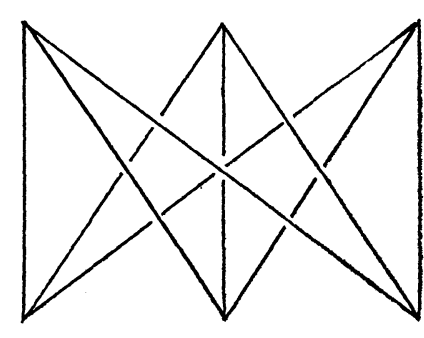

Fig. 2

The graph of this curve is Fig. 2. This is known to be the simplest graph without embedding into plane.

By $j$ the point corresponding to this curve is mapped to the point

$$
\lim _{t \rightarrow \infty} \sqrt{-1} t\left(\begin{array}{rrrr}
4 & 1 & -2 & -2 \\
1 & 4 & -2 & -2 \\
-2 & -2 & 4 & 1 \\
-2 & -2 & 1 & 4
\end{array}\right) \bmod . S p(4, Z)
$$

in $\widetilde{\mathfrak{S}}_{4}^{*}$. The integral matrix above is equal to $e_{12,345}+e_{34,125}$ in Igusa's notation [8] which is not conjugate to any points in $C_{\circ}$.

\section{\$ 8. The case of irreducible stable curves.}

In this section we shall study the generalized period matrices of irreducible stable curves. In the moduli space $\mathscr{S}_{g}$ of stable curves of genus $g$ those points which correspond to irreducible stable curves form an open subset $\mathscr{U}_{g}$. Using the results on the period matrices of irreducible stable curves, we shall show finally that the canonical map $j: \mathscr{S}_{g} \rightarrow \widetilde{\Xi}_{g}^{*}$ is injective on $\mathscr{U}_{g}$.

(8.1) Let $C$ be an irreducible stable curve of genus $g$ with the double points $z_{1}, \cdots, z_{g^{\prime \prime}}$. Let $f: \tilde{C} \rightarrow C$ be the normalization of $C$ and $x_{i}, y_{i}$, $i=1, \cdots, g^{\prime \prime}$, the points in $\tilde{C}$ with $f\left(x_{i}\right)=f\left(y_{i}\right)=z_{i}$. The genus of $\tilde{C}$ is then $g^{\prime}=g-g^{\prime \prime}$. 
Choose a canonical basis $\left\{\alpha_{1}, \cdots, \alpha_{g^{\prime}}, \beta_{1}, \cdots, \beta_{g^{\prime}}\right\}$ of $H_{1}(\tilde{C}, Z)$ and let $\alpha_{g^{\prime}+i}, i=1, \cdots, g^{\prime \prime}$, be small circles in $\tilde{C}$ rounding $y_{i}$ once cunterclockwise and $\beta_{g^{\prime}+i}, i=1, \cdots, g^{\prime \prime}$, paths from $x_{i}$ to $y_{i}$ meeting $\alpha_{g^{\prime}+i}$ once but without meeting any other $\alpha$ 's and $\beta$ 's. Then the homology classes of the images of $\alpha_{1}, \cdots, \alpha_{g^{\prime}}, \beta_{1}, \cdots, \beta_{g^{\prime}}, \beta_{g^{\prime}+1}, \cdots, \beta_{g}$ by $f$ (which we denote by the same letters) form a canonical basis of $H_{1}(C, Z)$. Hence $H_{1}(C, Z)$ has rank $2 g^{\prime}+g^{\prime \prime}=2 g-g^{\prime \prime}$.

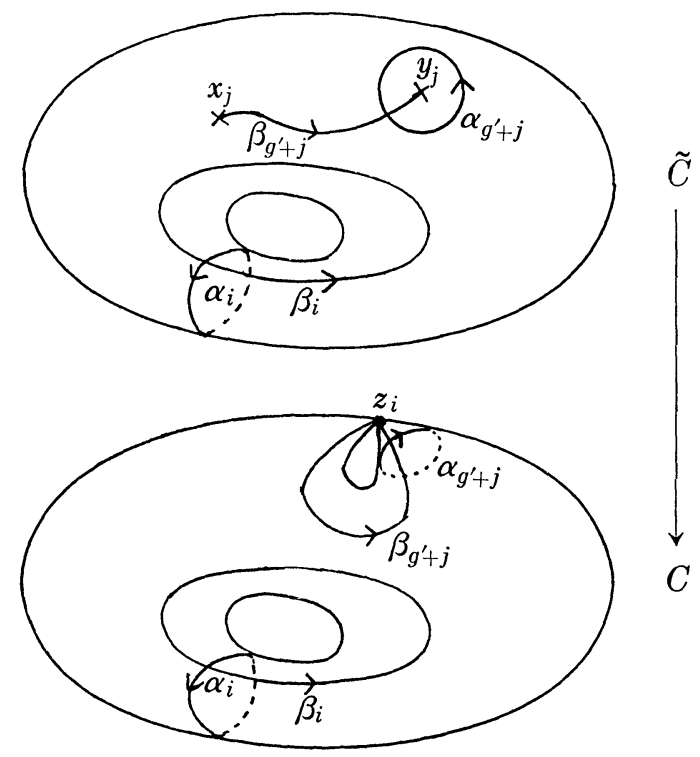

Next we shall choose a special basis of $\Gamma\left(C, \omega_{C}\right)$. Let $\omega_{1}^{\prime}, \cdots, \omega_{g}^{\prime}$, be $g^{\prime}$ independent holomorphic forms in $\tilde{C}$, that is, they form a basis of $\Gamma\left(\tilde{C}, \omega_{\tilde{C}}\right)$.

LEMMA 17. Let $D$ be a smooth curve of genus $\tilde{g}$ and $\{x, y\}$ a pair of two distinct points in $D$. Then there is a meromorphic form $\omega$ on $D$ which is holomorphic except on $x$ and $y$ and which has simple poles at $x$ and $y$.

Proof. Let $\omega_{D}(x+y)$ be the sheaf of meromorphic forms on $D$ except for simple poles at $x$ and $y$. Then we claim that

$$
\operatorname{dim}_{C} \Gamma(D, \omega(x+y))-\operatorname{dim}_{C} \Gamma\left(D, \omega_{D}\right)=1>0 .
$$

If a form $\omega$ has a simple pole at $x$, then $\omega$ must have a simple pole also at $y$ by virtue of the equality: $\operatorname{Res}_{x} \omega+\operatorname{Res}_{y} \omega=0$. Hence the lemma follows from the above claim. 
If $\tilde{g}$ is greater than 1 , we have $\operatorname{dim}_{C} \Gamma\left(D, \omega_{D}\right)=\tilde{g}$, and $\operatorname{deg} \omega_{D}=2 \tilde{g}$

-2 . On the other hand by the theorem of Riemann-Roch we have

$$
\begin{aligned}
\cdot \operatorname{dim}_{C} & \Gamma\left(D, \omega_{D}(x+y)\right) \\
& =-\operatorname{dim}_{C} \Gamma\left(D, \mathcal{O}_{D}(-x-y)\right)+\operatorname{deg} \omega_{D}(x+y)+1-\tilde{g} \\
& =0+2 \tilde{g}+1-\tilde{g}=\tilde{g}+1 .
\end{aligned}
$$

The other cases are similar.

q.e.d.

Hence for each pair $\left(x_{i}, y_{i}\right)$ we can choose a meromorphic form $\omega_{g^{\prime}+i}^{\prime}$ in $\Gamma\left(\tilde{C}, \omega_{\tilde{C}}\left(x_{i}+y_{i}\right)\right)$ with $\operatorname{Res}_{y_{i}} \omega_{g^{\prime}+i}^{\prime}=1$. Then $\omega_{1}^{\prime}, \cdots, \omega_{g^{\prime}}^{\prime}, \omega_{g^{\prime}+1}^{\prime}, \cdots, \omega_{g}^{\prime}$ defined above are clearly linearly independent, hence form a basis of $\Gamma\left(C, \omega_{C}\right)$.

(8.2) Let $A^{\prime}, B^{\prime}, C^{\prime}$ and $D^{\prime}$ be the matrices defined by

$$
\begin{aligned}
& A^{\prime}=\left(\int_{\alpha_{i}} \omega_{j}^{\prime}\right) \quad i, j=1, \cdots, g^{\prime}, B^{\prime}=\left(\int_{\beta_{i}} \omega_{j}^{\prime}\right) \quad i, j=1, \cdots, g^{\prime}, \\
& C^{\prime}=\left(\int_{\alpha_{i}} \omega_{g^{\prime}+j}^{\prime}\right) \begin{array}{l}
i=1, \cdots, g^{\prime} \\
j=1, \cdots, g^{\prime \prime}
\end{array}, \quad D^{\prime}=\left(\int_{\beta_{i}} \omega_{g^{\prime}+j}^{\prime}\right) \begin{array}{l}
i=1, \cdots, g^{\prime} \\
j=1, \cdots, g^{\prime \prime}
\end{array}
\end{aligned}
$$

Since $A^{\prime}$ is non-degenerate, we put $A^{\prime-1}=\left(\alpha_{i j}^{\prime \prime}\right)$. Put

$$
\omega_{j}=\sum_{k=1}^{g^{\prime}} a_{k j}^{\prime \prime} \omega_{k}^{\prime}, \quad 1 \leq j \leq g^{\prime},
$$

and

$$
\omega_{g^{\prime}+j}=\omega_{g^{\prime}+j}^{\prime}-\sum_{k, \ell=1}^{g^{\prime}} a_{k \ell}^{\prime \prime} c_{\ell j}^{\prime} \omega_{k}^{\prime}, \quad 1 \leq j \leq g^{\prime \prime}
$$

Then $\omega_{j}, 1 \leq j \leq g^{\prime}$, form a basis of $\Gamma\left(\tilde{C}, \omega_{\tilde{C}}\right)$ and $\omega_{g^{\prime}+j}$ is holomorphic except for simple poles at $x_{i}$ and $y_{i}$ and has its residue 1 at $y_{i}$. Hence we can replace the basis $\left\{\omega_{1}^{\prime}, \cdots, \omega_{g}^{\prime}\right\}$ of $\Gamma\left(C, \omega_{C}\right)$ by $\left\{\omega_{1}, \cdots, \omega_{g}\right\}$. If we denote by $B$ and $D$ the matrices

$$
\left(\int_{\beta_{i}} \omega_{j}\right) i, j=1, \cdots, g^{\prime}, \quad \text { and } \quad\left(\int_{\beta_{i}} \omega_{g^{\prime}+j}\right) \begin{aligned}
& i=1, \cdots, g^{\prime} \\
& j=1, \cdots, g^{\prime \prime}
\end{aligned},
$$

respectively, then we have $B=B^{\prime} A^{\prime-1}$ and $D=D^{\prime}-B^{\prime} A^{\prime-1} C^{\prime}$. Hence we have obtained

LEMMA 18. We can choose a basis $\left\{\omega_{1}, \cdots, \omega_{g}\right\}$ of $\Gamma\left(C, \omega_{C}\right)$ such that i) $\omega_{1}, \cdots, \omega_{g}$ are everywhere holomorphic on $\tilde{C}$ and form a basis of $\Gamma\left(\tilde{C}, \omega_{\tilde{C}}\right)$; 
ii) for each $i, 1 \leq i \leq g^{\prime \prime}, \omega_{g^{\prime}+i} i s$ holomorphic except for simple poles at $x_{i}$ and $y_{i}$ and has residue 1 at $y_{i}$;

iii)

$$
\int_{\alpha_{i}} \omega_{j}= \begin{cases}1 & \text { if } i=j \\ 0 & \text { if } i \neq j .\end{cases}
$$

For later use we put $E^{(n)}=\left(E_{i j}^{(n)}\right)_{1 \leq i<j \leq g^{\prime \prime}+1}$ with

$$
E_{i j}^{(n)}=\mathbf{e}\left((1 / n) \int_{\beta_{g,+}} \omega_{g^{\prime}+j}\right), \quad 1 \leq i<j \leq g^{\prime \prime},
$$

and

$$
E_{i j}^{(n)}=0, j=g^{\prime \prime}+1 .
$$

Remark. i) This basis is determined uniquely.

ii) We have an extension of Riemann's equality:

$$
\int_{\beta_{i}} \omega_{j}=\int_{\beta_{j}} \omega_{i}, \quad i, j=1, \cdots, g,
$$

except for $i=j=g^{\prime}+1, \cdots, g$.

In case of $i, j \leq g^{\prime}$ this is the usual Riemann's equality on $\tilde{C}$. The other cases are known to be the law of interchange of argument and parameter (cf. [29] §16).

(8.3) Let $e_{i}$ (resp. $\tilde{e}_{i}$ ) be the unit vector in $C^{g}$ (resp. in $C^{g^{\prime}}$ ) whose $i$-th coefficient is 1 and the others are 0 . Let $e_{g+i}\left(\operatorname{resp} . \tilde{e}_{g^{\prime}+i}\right)$ be the $i$-th column vector of the matrix $\left(\begin{array}{c}B \\ { }^{t} D\end{array}\right)$ (resp. $B$ ). Then the generalized Jacobian variety of $C$ is defined to be the group variety

$$
J(C)=C^{g} / \sum_{i=1}^{2 g^{\prime}+g^{\prime \prime}} Z e_{i},
$$

and the Jacobian variety of $\tilde{C}$ to be the torus

$$
J(\tilde{C})=\boldsymbol{C}^{g^{\prime}} / \sum_{i=1}^{2 g^{\prime}} \boldsymbol{Z} \tilde{e}_{i} .
$$

There is a canonical holomorphic group epimorphism $J(C) \rightarrow J(\tilde{C})$, whose kernel is a product of $g^{\prime \prime}$ copies of the multiplicative group $C^{*}$.

$$
0 \longrightarrow\left(C^{*}\right)^{g^{\prime \prime}} \longrightarrow J(C) \longrightarrow J(\tilde{C}) \longrightarrow 0 \text { (exact) . }
$$

That is $J(C)$ is a group extension of $J(\tilde{C})$ by $\left(C^{*}\right)^{g^{\prime \prime}}$. Hence $J(C)$ defines an extension class $e$ in $\operatorname{Ext}\left(J(\tilde{C}),\left(C^{*}\right)^{g^{\prime \prime}}\right)=\operatorname{Ext}\left(J(\tilde{C}),\left(C^{*}\right)\right)^{g^{\prime \prime}}$.

On the other hand it is known (e.g. [23]) that there is a canonical isomorphism : 


$$
\operatorname{Ext}\left(J(\tilde{C}), C^{*}\right) \stackrel{\sim}{\longrightarrow} \operatorname{Pic}^{0}(J(\tilde{C})) \stackrel{\sim}{\longrightarrow} J(\tilde{C}) .
$$

Proposition 7. Let $\left[d_{i}\right]$ denote the points in $J(\tilde{C})$ corresponding to the column vectors $d_{i}, i=1, \cdots, g^{\prime \prime}$, of $D$ in $C^{g^{\prime}}$ respectively. Then through the isomorphism (8.3.4) the extension class e of (8.3.3) in $\operatorname{Ext}\left(J(\tilde{C}),\left(C^{*}\right)^{g^{\prime \prime}}\right)$ corresponds to $\left(\left[d_{1}\right], \cdots,\left[d_{g^{\prime \prime}}\right]\right)$ in $J(\tilde{C})^{g^{\prime \prime}}$.

Proof. First of all we shall express the (inverse) isomorphism (8.3.4) explicitely. Denote by $Z$ the additive group $\sum Z \tilde{e}_{i}$ in $\boldsymbol{C}^{g^{\prime}}$.

Take a vector $d=\left(d_{i}\right)$ in $C^{g^{\prime}}$. Then we can define a homomorphism $f_{d}: Z \rightarrow C^{*}$ by sending $\tilde{e}_{i}$ to 1 for $1 \leq i \leq g^{\prime}$ and to $\mathbf{e}\left(d_{i-g^{\prime}}\right)$ for $g^{\prime}<i \leq 2 g^{\prime}$. By this homomorphism, define the action of $Z$ on $C^{*} \times C^{g^{\prime}}$ by

$$
\begin{aligned}
z: C^{*} \times C^{g^{\prime}} & \longrightarrow C^{*} \times C^{g^{\prime}} \\
(a, \zeta) & \longrightarrow\left(f_{d}(z) a, \zeta+z\right)
\end{aligned}
$$

for $z \in Z$. Hence we obtain a principal $C^{*}$-bundle:

$$
0 \longrightarrow C^{*} \longrightarrow C^{*} \times C^{g^{\prime}}\left|Z \longrightarrow C^{g^{\prime}}\right| Z=J(\tilde{C}) \longrightarrow 0 \text { (exact) . }
$$

By corresponding $d$ to the extension class of this $C^{*}$-bundle, we get a homomorphism: $C^{g^{\prime}} \rightarrow \operatorname{Ext}\left(J(\tilde{C}), C^{*}\right)$. (Clearly this correspondence is additive.) Moreover if $d$ is contained in $Z$, i.e. $d=\sum n_{i} \tilde{e}_{i}$ with $n_{i} \in Z$, then by the isomorphism:

$$
\begin{aligned}
C^{*} \times C^{g^{\prime}} & \sim C^{*} \times C_{\underset{\mathcal{E}}{g^{\prime}}}^{\sim} \\
\left(a, z_{i}\right) & \longrightarrow\left(\mathbf{e}\left(\sum_{j=g^{\prime}+1}^{2 g^{\prime}} n_{j} z_{j}\right) a, z_{i}\right)
\end{aligned}
$$

transforms the $G$-action above into the trivial one on $C^{*}$. (We use the fact the period matrix $\left(\tilde{e}_{g^{\prime}+1}, \cdots, \tilde{e}_{2 g^{\prime}}\right)$ is symmetric.) Hence the extension class corresponding to $d$ in $Z$ is trivial, i.e. the homomorphism above factors through $i: J(\tilde{C})=C^{g^{\prime}} / Z \rightarrow \operatorname{Ext}\left(J(\tilde{C}), C^{*}\right)$. This map $i$ gives the inverse of (8.3.4).

Now we are ready to prove the Proposition 7. If we take a quotient of $\boldsymbol{C}^{g}$ by the subgroup $Z_{1}=\sum_{i=1}^{g^{\prime \prime}} \boldsymbol{Z} e_{g^{\prime}+i}$, then this is isomorphic to $\boldsymbol{C}^{g^{\prime}} \times\left(\boldsymbol{C}^{*}\right)^{g^{\prime \prime}}$ by corresponding $\left(\zeta_{1}, \cdots, \zeta_{g}\right) \bmod Z_{1}$ to $\left(\zeta_{1}, \ldots, \zeta_{g^{\prime}}, \mathbf{e}\left(\zeta_{g^{\prime}+1}\right), \cdots, \mathbf{e}\left(\zeta_{g}\right)\right)$. Through the canonical isomorphism $\sum_{i=1}^{2 g^{\prime}+g^{\prime \prime}} Z e_{i} / Z_{1} \widetilde{\rightarrow}$, an action of $Z$ on $\boldsymbol{C}^{g^{\prime}} \times\left(\boldsymbol{C}^{*}\right)^{g^{\prime \prime}}$ is induced canonically as

$$
\begin{aligned}
z: C^{g^{\prime}} \times\left(C^{*}\right)^{g^{\prime \prime}} & \longrightarrow C^{g^{\prime}} \times\left(C^{*}\right)^{g^{\prime \prime}} \\
& \left(\zeta, a_{1}, \cdots, a_{g^{\prime \prime}}\right) \longrightarrow\left(\zeta+z, f_{d_{1}}(z) a_{1}, \cdots, f_{d_{g^{\prime \prime}}}(z) a_{g^{\prime \prime}}\right)
\end{aligned}
$$


for $z \in Z$, and with the action we have $J(C) \widetilde{\rightarrow} C^{g^{\prime}} \times\left(C^{*}\right)^{g^{\prime \prime}} / Z$, hence the conclusion follows.

q.e.d.

We can define a canonical embedding of $\tilde{C}$ into $J(\tilde{C})$. Fix a point $q$ in $\tilde{C}$. Then the embedding $i: \tilde{C} \rightarrow J(\tilde{C})$ is defined by

$$
\begin{aligned}
i: & \tilde{C} \longrightarrow J(\tilde{C}) \\
& \mathbb{\Psi} \\
& p \longrightarrow{ }^{t}\left(\int_{q}^{p} \omega_{1}, \cdots, \int_{q}^{p} \omega_{g^{\prime}}\right) \bmod Z .
\end{aligned}
$$

Since the cycle $\beta_{g^{\prime}+i}$ is the image of a path from $x_{i}$ to $y_{i}$ where $x_{i}$ and $y_{i}$ correspond to a double point $z_{i}$ in $C$, and since the $i$-th column vector $d_{i}$ of $D$ is equal to $\left(\int_{\beta_{g^{\prime}+i}} \omega_{j}\right)_{j=1, \cdots, g^{\prime}}$, we have

$$
\left[d_{i}\right]=i\left(y_{i}\right)-i\left(x_{i}\right) \text {. }
$$

Together with Proposition 7 we have obtained the following theorem.

THEOREM 6. Let $C$ be an irreducible stable curve of genus $g$ with the double points $z_{i}, i=1, \cdots, g^{\prime \prime}$. Let $\tilde{C}$ be the normalization of $C$ with genus $g^{\prime}=g-g^{\prime \prime}$, and $x_{i}, y_{i}$ the points in $\tilde{C}$ corresponding to $z_{i}$ Denote by $i$ the canonical embedding of $\tilde{C}$ into the Jacobian variety (8.3.5). Then the generalized Jacobian variety of $C$ is an extension of $J(\tilde{C})$ by $\left(C^{*}\right)^{g^{\prime \prime}}$ whose extension class correspond to $\left(i\left(y_{1}\right)-i\left(x_{1}\right), \cdots, i\left(y_{g^{\prime \prime}}\right)-i\left(x_{g^{\prime \prime}}\right)\right)$ in $J(\tilde{C})^{g^{\prime \prime}}$ through the isomorphism (8.3.4).

Remark. The extension class above in $J(\tilde{C})^{g^{\prime \prime}}$ is determined up to changing factors and the isomorphisms of $J(\tilde{C})$ as a principally polarized abelian variety.

(8.4) Now we are ready to prove the main result in this section.

THEOREM 7. The canonical map $j: \mathscr{S}_{g} \rightarrow \widetilde{\Xi}_{g}^{*}$ is injective on the open set $\mathscr{U}_{g}$ of points corresponding to irreducible stable curves.

The rest of this section is devoted to the proof of this theorem.

Take a point $x$ in $\mathscr{H}_{g}$ corresponding to an irreducible stable curve $C$. Choose a coordinate neighbourhood $U$ of $x$ with centre $x$ satisfying (3.4). In the same way as (6.2) take a ramified covering $V$ of $U$. Here we may assume $n=3$. If we define the period map $T=\left(T^{\prime}, T^{\prime \prime \prime}, T^{\prime \prime}\right)$ of the canonical family of stable curves on $V$ by using the canonical basis of $H_{1}(C, Z)$ and that of $\Gamma\left(C, \omega_{C}\right)$ introduced in (8.2) and (8.3), then $T$ is subject to 


$$
T(s)=\sum_{i=1}^{g^{\prime \prime}} \frac{\log s}{2 \pi \sqrt{-1}} 3 E_{g^{\prime}+i} S(s)
$$

where $E_{j}$ is the matrix with the $(j, j)$ coefficient 1 and 0 otherwise, and $S(s)$ is holomorphic on the whole $V$ (cf. Proof of Proposition 5.). Moreover by Lemma 12 in (4.6) we have $T^{\prime}(0)=A, T^{\prime \prime \prime}(0)=D$ and $\lim _{s \rightarrow 0} \mathbf{e}\left((1 / 3)\left(-T^{\prime \prime}(s)\right)\right)=E^{(3)}$. We denote $E^{(3)}$ simply by $E$.

Then as we have seen in the proof of Theorem 4, this period map $T$ induces a holomorphic map

$$
\hat{T}^{*}: V \longrightarrow \hat{\subseteq}_{g}^{*}(3)
$$

Moreover by Theorem (2.5) the image $p=\hat{T}^{*}(0)$ is a simple point in $\hat{\Im}_{g}^{*}(3)$ and a system of local coordinates with centre $p$ is given by

$$
\left(\tau^{\prime}-A, \zeta-D, \xi-E\right) \text {. }
$$

With this system of coordinates, $\hat{T}^{*}$ is expressed in the form

$$
\begin{aligned}
\hat{T}^{*}: V & \longrightarrow \hat{\mathfrak{S}}_{g}^{*}(3) \\
\underset{\cup}{U} & \stackrel{\Psi}{(s)} \longrightarrow\left(T^{\prime}(s)-A, T^{\prime \prime \prime}(s)-D, \mathbf{e}\left(-(1 / 3) T^{\prime \prime}(s)\right)-E\right) .
\end{aligned}
$$

The point $p$ being simple, we can identify $\hat{\subseteq}_{g}^{*}(3)$ with $\widetilde{\subseteq}_{g}^{*}(3)$ near $p$.

Now let $x_{1}$ and $x_{2}$ be two points in $\mathscr{H}_{g}$ corresponding to irreducible stable curves with $\tilde{j}\left(x_{1}\right)=\tilde{j}\left(x_{2}\right)$ where $\tilde{j}=j \circ p: \mathscr{H}_{g} \rightarrow \mathscr{S}_{g} \rightarrow \widetilde{\Xi}_{g}^{*}$. For each $i=1,2$ we can make the argument above and we shall use the same notations but with subscript or superscript $i$. Then we have $p_{1}=M \cdot p_{2}$ for an element $M$ in $S p(g, Z)$ by Proposition 2 . We shall give an explicit form of $M$ and its action.

Since $\bar{j}\left(p_{1}\right)=\bar{j}\left(p_{2}\right)$ is contained in the image of the Siegel operator $\Phi: \widetilde{\Im}_{g^{\prime}}^{*} \rightarrow \bar{\Im}_{g}^{*}$ where $\bar{j}=p \cdot \tilde{j}: \mathscr{H}_{g} \rightarrow \widetilde{\Im}_{g}^{*} \rightarrow \bar{\Im}_{g}^{*}, M$ can be expressed in the form

$$
\left(\begin{array}{cccc}
A^{\prime} & 0 & B^{\prime} & B_{1} \\
A_{1} & U & B_{2} & B_{3} \\
C^{\prime} & 0 & D^{\prime} & D_{1} \\
0 & 0 & 0 & { }^{t} U^{-1}
\end{array}\right)
$$

where $\quad M=\left(\begin{array}{ll}A^{\prime} & B^{\prime} \\ C^{\prime} & D^{\prime}\end{array}\right) \in S p\left(g^{\prime}, Z\right), U \in G L\left(g^{\prime \prime}, Z\right)$ and these matrices satisfy the equalities: $A_{1}{ }^{t} D^{\prime}-B_{2}{ }^{t} C^{\prime}+U^{t} D_{1}=0, A_{1}{ }^{t} B^{\prime}-B_{2}{ }^{t} A^{\prime}+U^{t} B_{1}=0$ and $\left(A_{1}{ }^{t} B_{2}+U^{t} B_{3}\right)-\left(B_{2}{ }^{t} A_{1}+B_{3}{ }^{t} U\right)=0$.

The action of $M$ on $\widetilde{S}_{g}$ is written explicitly as $\tau=\left(\tau^{\prime}, \zeta, \tau^{\prime \prime}\right) \rightarrow M \cdot \tau$ 
$=\left(M^{\prime} \cdot \tau^{\prime},\left(-\left(M^{\prime} \cdot \tau^{\prime}\right)\left(C^{\prime} \zeta+D_{1}\right)+\left(A^{\prime} \zeta+B_{1}\right){ }^{t} U,(M \cdot \tau)^{\prime \prime}=\left(-\left(A_{1} \tau^{\prime}+U^{t} \zeta+\right.\right.\right.\right.$ $\left.\left.B_{2}\right)\left(C^{\prime} \zeta+D_{1}\right)+\left(A_{1} \zeta+U \tau^{\prime \prime}+B_{3}\right){ }^{t} U\right)$. Note that $(M \cdot \tau)^{\prime \prime}=U \tau^{\prime \prime}{ }^{t} U+$ a function of $\left(\tau^{\prime}, \zeta\right)$.

Then the assumption: $p_{1}=M \cdot p_{2}$ implies that the imaginary part of the diagonal elements of $(M \cdot \tau)^{\prime \prime}$ tends to infinity when the diagonal elements of $\operatorname{Im} \tau$ tends to infinity by (8.4.1). Hence observing that $(M \cdot \tau)^{\prime \prime}$ $=U \tau^{\prime \prime}{ }^{t} U+$ a function of $\left(\tau^{\prime}, \zeta\right)$, we have $U^{t} U=1_{g^{\prime \prime}}$, i.e. $U=\left( \pm e_{\pi(1)}, \cdots\right.$, $\left.\pm e_{\pi\left(g^{\prime \prime}\right)}\right)$ for a permutation $\pi$ of $g^{\prime \prime}$ elements where $e_{i}$ is the unit vector with $i$-th coefficient 1 and 0 otherwise.

Hence if we replace the canonical basis of $H_{1}\left(\tilde{C}_{2}, Z\right)$ by $M^{\prime}\left\{\alpha^{(2)}, \beta^{(2)}\right\}$, change the index of $z_{i}^{(2)}$ to $z_{\pi(i)}^{(2)}$ and for every $i$ with $i$-th column vector of $U,-e_{\pi(i)}$, change $x_{\pi(i)}^{(2)}$ and $y_{\pi(i)}^{(2)}$, then we may assume that $M^{\prime}=1_{g^{\prime}}$ and $U=1_{g^{\prime \prime}}$.

Let us sum up our results obtained up to now.

(8.4.2) For an appropriate choice of the canonical basis of $H_{1}\left(C_{2}, Z\right)$ we may assume that $p_{1}=M \cdot p_{2}$ where $M$ is subjects to

$$
M=\left(\begin{array}{cccc}
1 & 0 & 0 & \mathfrak{n} \\
t_{\mathfrak{m}} & 1 & { }^{t} \mathfrak{n} & \mathfrak{s} \\
0 & 0 & 1 & -\mathfrak{m} \\
0 & 0 & 0 & 1
\end{array}\right)
$$

where $\mathfrak{m}$ and $\mathfrak{n}$ are integral $g^{\prime} \times g^{\prime \prime}$ matrices and $\mathfrak{g}$ is an integral $g^{\prime \prime} \times g^{\prime \prime}$ matrix with ${ }^{t} \mathfrak{m} \mathfrak{n}+{ }^{t} \mathfrak{g}={ }^{t} \mathfrak{n} \mathfrak{m}+\mathfrak{g}$. The action on $\mathbb{S}_{g}$ by $M$ is written as $\tau=\left(\tau^{\prime}, \zeta, \tau^{\prime \prime}\right) \rightarrow M \cdot \tau=\left(\tau^{\prime}, \zeta+\tau \mathfrak{m}+\mathfrak{n},(M \cdot \tau)^{\prime \prime}=\tau^{\prime \prime}+{ }^{t} \mathfrak{m} \tau^{\prime} \mathfrak{m}+\left({ }^{t} \mathfrak{m} \zeta+{ }^{t} \zeta \mathfrak{m}\right)\right.$ $\left.+\left({ }^{t} \mathfrak{m} \mathfrak{n}+{ }^{t} \mathfrak{g}\right)\right)$. Hence from the condition $p_{1}=M \cdot p_{2}$ we have

$$
\text { (**) }\left\{\begin{array}{l}
A_{1}=A_{2}=A, \\
D_{1}=D_{2}+A \mathfrak{m}+\mathfrak{n}, \\
\left.\left(E_{1}\right)_{i j}=\left(E_{2}\right)_{i j} \mathbf{e}\left((1 / 3)\left({ }^{t} \mathfrak{m} A \mathfrak{m}+\left({ }^{t} \mathfrak{m} D_{2}+{ }^{t} D_{2} \mathfrak{m}\right)+\left({ }^{t} \mathfrak{m} \mathfrak{n}\right)+{ }^{t} \mathfrak{g}\right)\right)_{i j}\right) .
\end{array}\right.
$$

If we define $J\left(C_{i}\right)$ and $J\left(\tilde{C}_{i}\right)$ with a basis of $H_{1}\left(C_{i}, Z\right)$ above as (8.3.1) and (8.3.2), then from the above equality we can identify $J\left(\tilde{C}_{1}\right)$ and $J\left(\tilde{C}_{2}\right)$, and the extension classes of $J\left(C_{1}\right)$ and $J\left(C_{2}\right)$ are the same through this identification. (End of (8.4.2.)).

Hence the following is the claim which should be proved.

(8.4.3) Let $\tilde{C}$ be a non-singular curve of genus $g^{\prime}$ and $\left(x_{j}^{(i)}, y_{j}^{(i)}\right), i=$ $1,2, j=1, \cdots, g^{\prime \prime}, 2 g^{\prime \prime}$ pairs of distinct elements in $\tilde{C}$ such that $\left(x_{j}^{(i)}, y_{j}^{(i)}\right)$ 
$\neq\left(x_{k}^{(i)}, y_{k}^{(i)}\right)$ for $j \neq k$. Denote by $C_{1}$ (resp. $C_{2}$ ) the curve obtained by identifying $x_{j}^{(1)}$ with $y_{j}^{(1)}$ (resp. $x_{j}^{(2)}$ with $y_{j}^{(2)}$ ), which is an irreducible stable curve of genus $g=g^{\prime}+g^{\prime \prime}$ with the double points $z_{j}^{(1)}$ (resp. $\left.z_{j}^{(2)}\right), j=1$, $\ldots, g^{\prime \prime}$, each of which corresponds to $x_{j}^{(1)}, y_{j}^{(1)}$ (resp. $\left.x_{j}^{(2)}, y_{j}^{(2)}\right)$ respectively. For each $i=1,2$ choose a canonical families of 1-cycles in $C_{i}$ and a basis of $\Gamma\left(C_{i}, \omega_{C_{i}}\right)$ such as (8.1) and (8.2) and define the matrices $A_{i}, D_{i}$, and $E_{i}$. If for an element $M$ of $S p(g, Z)$ of the form $(*)$ in (8.4.2) the above matrices are subject to $(* *)$ in (8.4.2), then $C_{1}$ and $C_{2}$ are isomorphic.

(8.5) We shall prove (8.4.3) by dividing it into the following four cases according to the properties of $\tilde{C}$. In every case if $C$ itself is non-singular, then Theorem 7 is the usual theorem of Torelli. Hence we assume that $C$ has at least one double point, i.e. $g^{\prime \prime}>0$.

A) The case that $\tilde{C}$ is a non-hyperelliptic curve with $g^{\prime}>2$.

The theorem follows from the following proposition and the last claim of (8.4.2).

Proposition 9. Let $C$ be an irreducible stable curve of genus g whose normalization is a non-hyperelliptic curve with genus $g^{\prime}>2$. Then $C$ is uniquely determined by its generalized Jacobian variety $J(C)$.

Proof. By Torelli's theorem the non-singular model $\tilde{C}$ is uniquely determind. Let $i: \tilde{C} \rightarrow J(\tilde{C})$ be the canonical embedding (8.3.5). Denote by $z_{i}, 1 \leq i \leq g^{\prime \prime}$, the double points of $C$ and by $x_{i}, y_{i}$ the corresponding points in $\tilde{C}$. Then by Theorem 6 the set of elements $i\left(y_{i}\right)-i\left(x_{i}\right), i=1$, $\cdots, g^{\prime \prime}$, is uniquely determined by $J(C)$ up to isomorphisms of $J(\tilde{C})$. Hence the proposition follows from the following lemma.

LEMMA 19. Let $C$ be a non-hyperelliptic curve and $J(C)$ the Jacobian variety of $C$. Define a canonical embedding $i: C \rightarrow J(C)$ as (8.3.5) Then the morphism $C \times C \rightarrow J(C)$ defined by sending $(x, y)$ to $i(x)-i(y)$ is injective outside the diagonal of $C \times C$.

Proof. Let $\left(x_{1}, y_{1}\right)$ and $\left(x_{2}, y_{2}\right)$ be two pairs of distinct points in $C$ with $i\left(x_{1}\right)-i\left(y_{1}\right)=i\left(x_{2}\right)-i\left(y_{2}\right)$. Then we have

$$
i\left(x_{1}\right)+i\left(y_{2}\right)=i\left(x_{2}\right)+i\left(y_{1}\right) .
$$

Hence by virtue of Abel's theorem the divisor $x_{1}+y_{2}$ is linearly equivalent to $x_{2}+y_{1}$. Assume that $x_{1}+y_{2} \neq x_{2}+y_{1}$. Then the above claim im- 
plies that $\operatorname{dim}_{C} H^{0}\left(C, \mathcal{O}\left(x_{1}+y_{2}\right)\right)>0$. Hence $C$ has a non-empty linear system of degree 2, i.e. $C$ is hyperelliptic. This contradicts with the assumption.

q.e.d.

B) The case that $\tilde{C}$ is a hyperelliptic curve with $g^{\prime}>1$.

The proof of Lemma 19 shows in fact the following.

LEMMA $19^{\text {bis. }}$. Let $C$ be a non-singular curve and $J(C)$ the Jacobian variety of $C$. Define a canonical embedding $i: C \rightarrow J(C)$ as (8.3.5). For two distinct pairs of distinct elements $\left(x_{1}, y_{1}\right)$ and $\left(x_{2}, y_{2}\right)$ in $C$, the equality: $i\left(x_{1}\right)-i\left(y_{1}\right)=i\left(x_{2}\right)-i\left(y_{2}\right)$ does hold if and only if the curve $C$ is hyperelliptic and $\iota\left(x_{1}\right)=y_{2}$ and $\iota\left(x_{2}\right)=y_{1}$ by the involution $\circ$ of $C$.

(We have proved only the "only if" part but the "if" part is clear.)

Now we shall prove (8.4.3) in case of a hyperelliptic curve $\tilde{C}$ by the induction on $g^{\prime \prime}$.

In case of $g^{\prime \prime}=1$, we have $i\left(y_{1}^{(1)}\right)-i\left(x_{1}^{(1)}\right)=i\left(y_{1}^{(2)}\right)-i\left(x_{1}^{(2)}\right)$ in $J(\tilde{C})$ by (8.4.2). If $\left\{x_{1}^{(1)}, y_{1}^{(1)}\right\}=\left\{x_{1}^{(2)}, y_{1}^{(2)}\right\}$, then there is nothing to prove. If they are different, then Lemma $19^{\text {bis }}$ shows that by the involution \& of $\tilde{C}$ the set $\left\{x_{1}^{(1)}, y_{1}^{(1)}\right\}$ is mapped to $\left\{x_{1}^{(2)}, y_{1}^{(2)}\right\}$. Hence this involution induces an isomorphism between $C_{1}$ and $C_{2}$.

In case of $g^{\prime \prime}=2$, we have

$$
i\left(y_{i}^{(1)}\right)-i\left(x_{i}^{(1)}\right)=i\left(y_{i}^{(2)}\right)-i\left(x_{i}^{(2)}\right), \quad i=1,2 .
$$

If $\left\{x_{i}^{(1)}, y_{i}^{(1)}\right\}=\left\{x_{i}^{(2)}, y_{i}^{(2)}\right\}$ for $i=1,2$ or $\left\{x_{i}^{(1)}, y_{i}^{(1)}\right\}=\iota\left(\left\{x_{i}^{(2)}, y_{i}^{(2)}\right\}\right)$ for $i=1,2$ with the involution $c$ of $\tilde{C}$, then clearly $C_{1}$ and $C_{2}$ are isomorphic. Hence we shall assume that $\iota\left(\left\{x_{1}^{(2)}, y_{1}^{(2)}\right\}\right)=\left\{x_{1}^{(1)}, y_{1}^{(1)}\right\}$ and $\left\{x_{2}^{(1)}, y_{2}^{(1)}\right\}=\left\{x_{2}^{(2)}, y_{2}^{(2)}\right\}$. In order to prove the assertion, we must strengthen the assumption (8.4.3). We add two more assumptions:

$(8.4 .3)^{\mathrm{bis}}$ in the family of 1-cycles on $\tilde{C}, \alpha_{i}^{(1)}=\alpha_{i}^{(2)}, \beta_{i}^{(1)}=\beta_{i}^{(2)}$ and $\omega_{i}^{(1)}$ $=\omega_{i}^{(2)}$ for $1 \leq i \leq g^{\prime}$; in the form of $M$ we assume moreover $\mathfrak{m}=0$.

Let us show that these assumptions can be satisfied. First of all note that if we take the same $\alpha_{i}^{(1)}=\alpha_{i}^{(2)}, \beta_{i}^{(1)}=\beta_{i}^{(2)}$ and $\omega_{i}^{(1)}=\omega_{i}^{(2)}, 1 \leq i \leq g^{\prime}$, then we have $A_{1}=A_{2}$. When we consider the form $M, M^{\prime}$ must be subject to $M^{\prime} \cdot A_{2}=A_{1}$. Since there is an automorphism of $\tilde{C}$ which induces $M^{\prime}$, we may assume $M^{\prime}=1_{g^{\prime}}$ by identifying $\tilde{C}_{1}=\tilde{C}$ and $\tilde{C}_{2}=\tilde{C}$ through this isomorphism (Here we used the condition that $\tilde{C}$ is hyperelliptic. In general cases we can reduce $M^{\prime}= \pm 1_{g^{\prime}}$ in the same way.). 
The other reduction of the form of $M$ in (8.4.2) does not concern with $\alpha_{i}$ 's and $\beta_{i}$ 's for $1 \leq i \leq g^{\prime}$. Hence we may assume the $\alpha_{i}^{(1)}=\alpha_{i}^{(2)}, \beta_{i}^{(1)}$ $=\beta_{i}^{(2)}$ and $\omega_{i}^{(1)}=\omega_{i}^{(2)}$ for $1 \leq i \leq g^{\prime}$.

Moreover if a column vector $\mathfrak{m}_{j}$ of $\mathfrak{m}$ is not zero, i.e. $d_{j}^{(1)}=d_{j}^{(2)}+$ $A \mathfrak{m}_{j} \neq d_{j}^{(2)}$ where $d_{j}^{(i)}$ is the $j$-th column vector of $D_{i}$, then $y_{j}^{(1)} \neq y_{j}^{(2)}$. In fact if $y_{j}^{(1)}=y_{j}^{(2)}$ and $\left[d_{j}^{(1)}\right]=\left[d_{j}^{(2)}\right]$, then we have $i\left(x_{j}^{(1)}\right)=i\left(x_{j}^{(2)}\right)$, hence $x_{j}^{(1)}=x_{j}^{(2)}$ and $d_{j}^{(1)}=d_{j}^{(2)}$. Hence we can move $\alpha_{i}=\alpha_{i}^{(1)}=\alpha_{i}^{(2)}$ to $\alpha_{i}^{\prime}$ so that $\alpha_{i}^{\prime}$ is homologous to $\alpha_{i}$ in $H_{1}\left(\tilde{C}-\left\{y_{j}^{(1)}\right\}, Z\right)$ and homologous to $\alpha_{i}+$ $m_{i j} \alpha_{g^{\prime}+j}$ in $H_{1}\left(\tilde{C}-\left\{y_{j}^{(2)}\right\}, Z\right)$. Then replacing $\alpha_{i}$ by $\alpha_{i}^{\prime}$, we can assume that $\mathfrak{m}_{j}=0$. Repeating this process for $j=1, \cdots, g^{\prime \prime}$, we can assume that $\mathfrak{m}=0$. Hence we can add the assumption $(8.4 .3)^{\text {bis }}$ to $(8.4 .3)$.

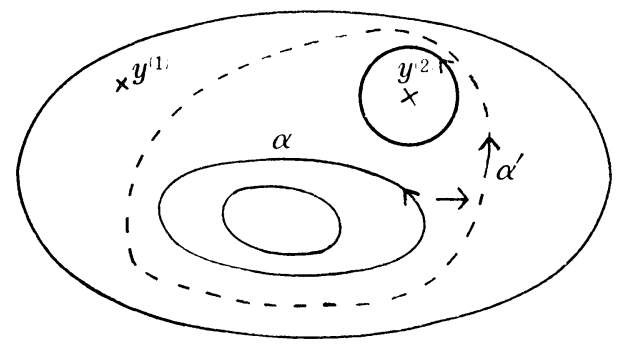

Now we shall go back to the proof of (8.4.3). Define multiple valued holomorphic functions $v_{i}(z)$ on $\tilde{C}$ as

$$
v_{i}(z)=\mathbf{e}\left(\int^{z} \omega_{g^{\prime}+1}^{(i)}\right), \quad i=1,2 .
$$

This function is a so-called multiplicative function, i.e. there is a character $\chi_{i}: \pi_{1}(\tilde{C}) \rightarrow C^{*}$ of the fundamental group of $\tilde{C}$ such that, for every closed path $\Gamma$ with base point $z$, the analytic continuation $v_{i}(\Gamma z)$ of $v_{i}(z)$ along $\Gamma$ is subject to

$$
v_{i}(\Gamma z)=\chi_{i}([\Gamma]) v_{i}(z)
$$

where $[\Gamma]$ is the homotopy class of $\Gamma$.

Note that the character $\chi_{1}$ is determined by the values $\chi_{i}\left(\left[\alpha_{j}\right]\right)$ and $\chi_{i}\left(\left[\beta_{j}\right]\right), 1 \leq j \leq g^{\prime}$. Since $\chi_{i}\left(\left[\alpha_{j}\right]\right)=\mathbf{e}\left(\int_{\alpha_{i}} \omega_{g^{\prime}+1}^{(i)}\right)=1$, and $\chi_{i}\left(\left[\beta_{j}\right]\right)=\mathbf{e}\left(\int_{\beta_{j}} \omega_{g^{\prime}+1}^{(i)}\right)$ $=\mathbf{e}\left(\left(D_{i}\right)_{j 1}\right)=\mathbf{e}\left(\left(d_{1}^{(1)}\right)_{j}\right)$, together with the assumption that $d_{1}^{(1)} \equiv d_{1}^{(2)} \bmod . Z^{g^{\prime}}$ (cf. $(8.4 .2)(* *)$ and $\left.(8.4 .3)^{\mathrm{bis}}\right)$, these characters coincide. Hence the ratio $f(z)=v_{1}(z) / v_{2}(z)$ is a (single-valued) meromorphic function on $\tilde{C}$. Moreover by the very definition $v_{i}(z)$ has a simple pole at $x_{1}^{(i)}$ and a simple 
zero at $y_{1}^{(i)}$ and it has neither poles nor zeros outside. Hence $f(z)$ has simple poles at $x_{1}^{(1)}$ and $y_{1}^{(2)}$ and simple zeros at $y_{1}^{(1)}$ and $x_{1}^{(2)}$ and it has neither poles nor zeros outside. Considering $f$ to be a holomorphic map from $\tilde{C}$ to $\boldsymbol{P}^{1}$, we see that this map is of degree 2 by the above observation, hence the non-trivial covering transformation of this covering $f$ gives the involution c of $\tilde{C}$.

On the other hand we have $\left(\left(E_{i}\right)_{12}\right)^{3}=v_{i}\left(y_{2}^{(i)}\right) / v_{i}\left(x_{2}^{(i)}\right)$ by definition and $\left(\left(E_{1}\right)_{12}\right)^{3}=\left(\left(E_{2}\right)_{12}\right)^{3}$ by (8.4.2) $\left.{ }^{* *}\right)$ and $(8.4 .3)^{\text {bis. }}$. Hence we have

$$
\frac{v_{1}\left(y_{2}^{(1)}\right)}{v_{1}\left(x_{2}^{(1)}\right)}=\frac{v_{2}\left(y_{2}^{(2)}\right)}{v_{2}\left(x_{2}^{(2)}\right)} \text {. }
$$

Since $x_{2}^{(1)}=x_{2}^{(2)}$ and $y_{2}^{(1)}=y_{2}^{(2)}$ by assumption, we have

$$
f\left(y_{2}^{(1)}\right)=v_{1}\left(y_{2}^{(1)}\right) / v_{2}\left(y_{2}^{(1)}\right)=v_{1}\left(x_{2}^{(1)}\right) / v_{2}\left(x_{2}^{(1)}\right)=f\left(x_{2}^{(1)}\right),
$$

i.e. $\iota\left(y_{2}^{(1)}\right)=x_{2}^{(1)}$. Hence the involution $\iota$ induces an isomorphism between $C_{1}$ and $C_{2}$.

In case of $g^{\prime \prime}>2$ the proof is similar, hence we shall omit it.

C) The case that $\tilde{C}$ is an elliptic curve, i.e. $g^{\prime}=1$.

The proof is similar to the case $B$ ). In this case we may also assume $(8.4 .3)^{\text {bis }}$ by the same reason as before.

In this case $J(\tilde{C})$ is isomorphic to $\tilde{C}$ by the canonical embedding $i$ (8.3.5), hence we identify them. The proof is done by induction on $g^{\prime \prime}$.

In case of $g^{\prime \prime}=1$, by Theorem 6 we have $y_{1}^{(1)}-x_{1}^{(1)}=y_{1}^{(2)}-x_{1}^{(2)}$. Hence the translation by $x_{1}^{(2)}-x_{1}^{(1)}$ maps $x_{1}^{(1)}$ to $x_{1}^{(2)}$ and $y_{1}^{(1)}$ to $y_{1}^{(2)}$, which induces an isomorphism between $C_{1}$ and $C_{2}$.

In case of $g^{\prime \prime}=2$, we also have the equalities: $y_{i}^{(1)}-x_{i}^{(1)}=y_{i}^{(2)}-x_{i}^{(2)}$ for $i=1,2$. By translation we may assume that $x_{2}^{(1)}=x_{2}^{(2)}=0$ and $y_{2}^{(1)}=y_{2}^{(2)}=y$.

Put $v_{i}(z)=\mathbf{e}\left(\int^{z} \omega_{2}^{(i)}\right), i=1,2$. Then they are multiple-valued holomorphic functions with characters $\chi_{i}: \pi_{1}(\tilde{C}) \rightarrow C^{*}$ such that for any closed path $\Gamma$ with base point $z$ the analytic continuation $v_{i}(\Gamma z)$ of $v_{i}(z)$ along $\Gamma$ is subject to

$$
v_{i}(\Gamma z)=\chi_{i}([\Gamma]) v_{i}(z)
$$

where $[\Gamma]$ is the homotopy class of $\Gamma$ in $\pi_{1}(\tilde{C})$. The only pole of $v_{i}(z)$ is a simple pole at $x_{1}^{(i)}$ and the only zero of $v_{i}(z)$ is a simple zero at $y_{1}^{(i)}$. 
If the assumptions in (8.4.3) and (8.4.3) ${ }^{\text {bis }}$ are satisfied, by the same argument as the case $\mathrm{B}), f(z)=v_{1}(z) / v_{2}(z)$ is a meromorphic function on $\tilde{C}$, and considered as a holomorphic map from $\tilde{C}$ to $\boldsymbol{P}^{1}$, it gives a two-fold covering with $f\left(x_{1}^{(1)}\right)=f\left(y_{1}^{(2)}\right)=\infty, f\left(y_{1}^{(1)}\right)=f\left(x_{1}^{(2)}\right)=0$ and moreover $f(y)=f(0)$ since $\left(\left(E_{1}\right)_{12}\right)^{3}=\left(\left(E_{2}\right)_{12}\right)^{3}$. Hence the non-trivial covering transformation induces the isomorphism between $C_{1}$ and $C_{2}$.

To prove the case $g^{\prime \prime}=3$ we note first of all that the above covering transformation $\iota$ is given explicitly as

$$
\begin{aligned}
\iota: \tilde{C} \longrightarrow & \tilde{C} \\
& \\
& z \longrightarrow
\end{aligned}
$$

(Note that $x_{1}^{(1)}+y_{1}^{(2)}=x_{1}^{(2)}+y_{1}^{(1)}$.) That is, if $\left(E_{12}^{(1)}\right)^{3}=\left(E_{12}^{(2)}\right)^{3}$, then

$$
x_{2}^{(1)}-x_{1}^{(1)}=x_{2}^{(2)}-x_{1}^{(2)}
$$

or

$$
x_{2}^{(1)}-x_{1}^{(1)}=-\left(y_{2}^{(2)}-y_{1}^{(2)}\right) .
$$

Therefore if $\left(E_{i j}^{(1)}\right)^{3}=\left(E_{i j}^{(2)}\right)^{3}$ for $1 \leq i, j \leq 3, i \neq j$ (we put $E_{j i}=E_{i j}$ if $i<j$ for the sake of convenience), then for each $(i, j)$ we have one of the following equalities:

$$
\begin{array}{ll}
\left(A_{i j}\right) & x_{j}^{(1)}-x_{i}^{(1)}=x_{j}^{(2)}-x_{i}^{(2)}, \\
\left(B_{i j}\right) & x_{j}^{(1)}-x_{i}^{(1)}=-\left(y_{j}^{(2)}-y_{i}^{(2)}\right) .
\end{array}
$$

Clearly if $\left(A_{i j}\right)$ and $\left(A_{j k}\right)$ hold, then $\left(A_{i k}\right)$ holds, and if $\left(B_{i j}\right)$ and $\left(B_{j k}\right)$ hold, then $\left(B_{i k}\right)$ holds. Hence for all $(i, j)$ either $\left(A_{i j}\right)$ or $\left(B_{i j}\right)$ does hold. If the equalities $\left(A_{i j}\right)$ hold for all $(i, j)$, then the translation of $\tilde{C}_{2}$ by $x_{1}^{(1)}-x_{1}^{(2)}$ induces an isomorphism between $C_{2}$ and $C_{1}$; if the equalities $\left(B_{i j}\right)$ hold for all $(i, j)$, then the involution above of $\tilde{C}_{2}$ induces an isomorphism between $C_{2}$ and $C_{1}$.

In case of $g^{\prime \prime}>3$ the proof is the same as the case of $g^{\prime \prime}=3$.

Remark (Ueno). We can also give these functions appeared above explicitly.

First of all we shall give the form of $\omega$ 's explicitly. For simplicity we assume that $g^{\prime \prime}=1$ and write $x$ and $y$ instead of $x_{1}$ and $y_{1}$. The curve $\tilde{C}$ is given as an elliptic curve $C / Z+Z \tau$ with $\operatorname{Im} \tau>0$. We shall 
denote by $z$ a uniformizing parameter of $\tilde{C}$, i.e. a coordinate of $C$ above. Put $\omega_{1}^{\prime}=d z$ and $\omega_{2}^{\prime}=(1 / 2 \pi \sqrt{-1})(\zeta(z-y)-\zeta(z-x)) d z$ where $\zeta(z)$ is the so-called zeta function of an elliptic curve defined as

$$
\zeta(z)=\frac{1}{z}+\sum_{(n, m) \neq(0,0)}\left(\frac{1}{z-m \tau-n}+\frac{z}{(m \tau+n)^{2}}+\frac{1}{m \tau+n}\right)
$$

Then it is easy to see that $\omega_{2}^{\prime}$ is a meromorphic form on $\tilde{C}$ with simple poles at $x$ and $y$ and with $\operatorname{Res}_{y} \omega_{2}^{\prime}=1$. Define $\alpha_{1}, \alpha_{2}, \beta_{1}, \beta_{2}$ as in (8.1). Then we have

$$
\begin{aligned}
& \int_{\alpha_{1}} \omega_{1}^{\prime}=1, \\
& \int_{\alpha_{2}} \omega_{1}^{\prime}=0, \\
& \int_{\beta_{1}} \omega_{1}^{\prime}=\tau
\end{aligned}
$$

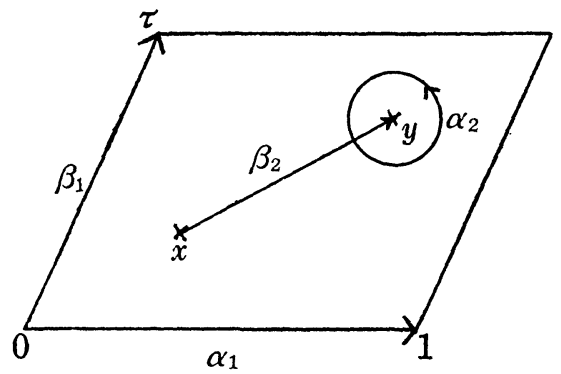

and by using the equality $\zeta(z)=\sigma^{\prime}(z) / \sigma(z)$ where $\sigma(z)$ is the $\sigma$-function defined as

$$
\sigma(z)=z \prod_{(m, n) \neq(0,0)}\left(1-\frac{z}{m \tau+n}\right) \exp \left(\frac{z}{m \tau+n}+\frac{1}{2}\left(\frac{z}{m \tau+n}\right)^{2}\right)
$$

and using the quasi-periodicity of $\sigma(z)$, i.e. the formula

$$
\sigma(z+m \tau+n)=(-1)^{m n+m+n}\left[\exp \left(\left(2 n \eta_{1}+2 m \eta_{2}\right)\left(z+\frac{n}{2}+\frac{m \tau}{2}\right)\right)\right] \sigma(z)
$$

where $\eta_{1}=\zeta(1 / 2)$ and $\eta_{2}=\zeta(\tau / 2)$, we have

$$
\begin{aligned}
& \int_{\alpha_{1}} \omega_{2}^{\prime}=\frac{\eta_{1}}{2 \pi \sqrt{-1}}(x-y), \\
& \int_{\alpha_{2}} \omega_{2}^{\prime}=1,
\end{aligned}
$$




$$
\int_{\beta_{1}} \omega_{2}^{\prime}=\frac{\eta_{2}}{2 \pi \sqrt{-1}}(x-y)
$$

Hence if we put $\omega_{1}=\omega_{1}^{\prime}, \omega_{2}=\omega_{2}^{\prime}-\left(\eta_{1}(-y) / \pi \sqrt{-1}\right) \omega_{1}^{\prime}$, then with the Legendre relation: $\eta_{1} \tau-\eta_{2}=\pi \sqrt{-1} / 2$ we can see that they form the basis introduced in Lemma 18, and we have $\int_{\beta_{1}} \omega_{2}=y-x$.

Then the multiplicative function we have introduced above is written explicitly as

$$
\begin{aligned}
v(z) & =\mathbf{e}\left(\int^{z} \omega_{2}\right) \\
& =\exp \left(2 \pi \sqrt{-1} \int^{z}\left(\omega_{2}^{\prime}-\frac{\eta_{1}}{\pi \sqrt{-1}}(x-y) \omega_{1}^{\prime}\right)\right) \\
& =\frac{\sigma(z-y)}{\sigma(z-x)} \exp \left(2 \eta_{1}(y-x) z\right) .
\end{aligned}
$$

Hence $E_{i j}^{3}$ is given explicitly as

$$
E_{i j}^{3}=\frac{\sigma\left(y_{i}-y_{j}\right) \sigma\left(x_{i}-x_{j}\right)}{\sigma\left(y_{i}-x_{j}\right) \sigma\left(x_{i}-y_{j}\right)} \exp \left(2 \eta_{1}\left(y_{i}-x_{i}\right)\left(y_{j}-x_{j}\right)\right) .
$$

About the formulae used above we refer the reader to [7] 2. Abschnitt, 1. Kapital for example.

D) The case that $\tilde{C}$ is rational, i.e. $g^{\prime}=0$.

Then clearly $g^{\prime \prime}=g>1$. The form of $M$ in (8.4.2) (*) is very simply

$$
M=\left(\begin{array}{cc}
1_{g} & \mathfrak{g} \\
0 & 1_{g}
\end{array}\right)
$$

with an integral symmetric matrix $\mathfrak{g}$ of degree $g$ and only the matrix $E$ appears.

First of all we shall give $\omega_{i}$ and $E$ explicitly. Let $C$ be a stable curve obtained by identifying $x_{i}$ and $y_{i}, 1 \leq i \leq g$, in a projective line $\tilde{C}=\boldsymbol{P}^{1}$. Denote by $z_{i}$ the double point corresponding to $x_{i}$ and $y_{i}$. Let $z$ be an inhomogeneous coordinate of $\tilde{C}$. Then the meromorphic forms: $\omega_{i}=\left(1 / 2 \pi \sqrt{-1)}\left(\left(1 /\left(z-y_{i}\right)\right)-\left(1 /\left(z-x_{i}\right)\right)\right) d z, 1 \leq i \leq g\right.$, form a basis of $\Gamma\left(C, \omega_{C}\right)$ in Lemma 18 , and by integrating them we have

$$
\int_{\beta_{i}} \omega_{j}=\frac{1}{2 \pi \sqrt{-1}} \log \frac{\left(y_{i}-y_{j}\right)\left(x_{i}-x_{j}\right)}{\left(x_{i}-y_{j}\right)\left(y_{i}-x_{j}\right)}
$$

hence 


$$
E_{i j}^{3}=\frac{\left(y_{i}-y_{j}\right)\left(x_{i}-x_{j}\right)}{\left(x_{i}-y_{j}\right)\left(y_{i}-x_{j}\right)}
$$

By (8.4.2) we have $\left(E_{i j}^{(1)}\right)^{3}=\left(E_{i j}^{(2)}\right)^{3}$ for all $1 \leq i<j \leq g$. Hence it is sufficient to prove that

$\left(^{*}\right)$ the family of values $\left(E_{i j}^{3}\right)_{1 \leq i<j \leq g}$ determines the isomorphism class of $C$ uniquely.

We use the induction on $g$.

In case of $g=2$. we may assume that $x_{1}=0, y_{1}=\infty$ and $y_{2}=1$ by changing $z$ with a projective linear transformation. Then with the equality above we have

$$
E_{12}^{3}=\frac{(\infty-1)\left(0-x_{2}\right)}{(0-1)\left(\infty-x_{2}\right)}=x_{2} .
$$

Hence the claim $\left(^{*}\right)$ is true for $g=2$.

In case of $g=3$, we may also assume that $x_{1}=0, y_{1}=\infty$ and $y_{2}=$ 1 as above. Then we have

$$
\begin{aligned}
& E_{12}^{3}=x_{2}, \\
& E_{13}^{3}=x_{3} / y_{3}, \\
& E_{23}^{3}=\frac{\left(1-y_{3}\right)\left(x_{2}-x_{3}\right)}{\left(1-x_{3}\right)\left(x_{2}-y_{3}\right)} .
\end{aligned}
$$

Solving these equations, we have the unique solution for $x_{2}$ and two pairs of solutions $\left(x_{3}^{(i)}, y_{3}^{(i)}\right), i=1,2$, for $\left(x_{3}, y_{3}\right)$. However a linear transformation $\rho: z \rightarrow x_{2} / z$ preserves the sets $\left\{x_{1}, y_{1}\right\}$ and $\left\{x_{2}, y_{2}\right\}$. If we put $x_{3}^{\prime}=\rho\left(y_{3}\right)=x_{2} / y_{3}$ and $y_{3}^{\prime}=\rho\left(x_{3}\right)=x_{2} / x_{3}$, then it is easy to see that the transformation $\left(x_{3}, y_{3}\right) \rightarrow\left(x_{3}^{\prime}, y_{3}^{\prime}\right)$ sends a set of solution for $\left(x_{3}, y_{3}\right)$ to another. By an elementary calculation we can see that $x_{3}=x_{3}^{\prime}$ if and only if the above pairs of solutions coincide. Hence the isomorphism class of $C$ is uniquely determined by the data, $E_{12}^{3}, E_{13}^{3}$ and $E_{23}^{3}$.

In case of $g>3$ the proof is similar, hence we omit it.

Thus we have proved (8.4.2) in every case A), B), C) and D), hence the proof of Theorem 7 is now complete.

(8.6) Remark. Assume that $g>2$. Let $\mathscr{N}$ be the union of divisors $\mathscr{N}_{i}, 1 \leq i \leq[\mathrm{g} / 2]$ whose general points correspond to stable curves with two non-singular irreducible components $C_{j}, j=1,2$, with genus $i$ and 
$g-i$ meeting at one point. Then $j$ is not injective on $\mathscr{N}$. In fact by the upper semicontinuity of the dimension of fibres, we have only to prove it for general points $\xi$ on $\mathscr{N}_{i}$. Hence the image by $j$ is contained in $\mathfrak{S}_{g}^{*}$, so it is sufficient to show that $\bar{j}$ is not injective. Let $C$ be the corresponding stable curve with the irreducible components $C_{1}$ and $C_{2}$. Let $x, y$ be the points on $C_{1}$ and $C_{2}$ respectively which coincide on $C$. Since $g>2$, we have $g-i>1$, i.e. the automorphism group of $C_{2}$ is finite. On the other hand if two such curves $C^{(1)}$ and $C^{(2)}$ are isomorphic, then it induces an isomorphism between $C_{2}^{(1)}$ and $C_{2}^{(2)}$ which maps $y^{(1)}$ to $y^{(2)}$. (In case of $i=g / 2$ we may need to change factors.) Hence the curves $C$ with different $y$ 's are not isomorphic in general. However, their generalized Jacobians are both $J(C)=J\left(C_{1}\right) \times J\left(C_{2}\right)$, hence the images by $\bar{j}$ coincide. This proves the assertion.

It is naturally expected that $j$ is injective outside of $\mathcal{N}$, but we have no proof.

\section{§. The case of $g=2$.}

(9.1) In this section we shall prove the following theorem.

THEOREM 8. In case of $g=2$ the canonical morphism $j: \mathscr{S}_{2} \rightarrow \overline{\mathfrak{S}}_{2}^{*}$ (Corollary of Theorem 4) is an isomorphism.

Since these two varieties are normal and complete, and since $j$ is birational, we have only to prove that $j$ has no fibres with positive dimension by virtue of Zariski's main theorem.

(9.2) The explicit structure of $\widetilde{\varsigma}_{2}^{*}\left(\right.$ or $\widetilde{\Im}_{2}^{*}(n)$ ) is known ([8], [18]). In fact this was the first compactification of $\mathfrak{S}_{2}^{*}$ constructed by Satake (ibid.).

So we must study the structure of the moduli space $\mathscr{S}_{2}$ of stable curves of genus 2 .

Proposition 10. Every stable curve of genus two is of one of the following types. Any curves of the same type are homeomorphic to each other. Those points in $\mathscr{S}_{2}$ which correspond to each type of stable curves form a locally closed algebraic subset in $\mathscr{S}_{2}$, hence making them strata, we can define a stratification in $\mathscr{S}_{2}$.

Type The corresponding stable curve

I a non-singular curve of genus 2

rank $H_{1}(C, Z)$ Stratum 
II $\quad$ a join of two non-singlar elliptic

curves meeting at one point transversally.

$\mathrm{III}_{a)}$ an elliptic curve with an ordinary double point.

$\mathrm{III}_{b\rangle} \quad a$ join of a non-singular elliptic

3

$\mathscr{B}_{a)}$ curve and a rational curve with an ordinary double point meeting at one point transversally.

$\mathrm{IV}_{a} \quad$ a rational curve with two ordinary double points.

$\mathrm{IV}_{b)} \quad$ a join of two rational curves with an ordinary double point meeting at one point transversally.

$\mathrm{V} \quad a$ join of two non-singular rational curves meeting at three points transversally.

The proof is straightforward, and we omit it (cf. [14] or [15]).

It is also easy to see that the holomorphic map $\bar{j}=p \circ j: \mathscr{S}_{2} \rightarrow \widetilde{\Xi}_{2}^{*}$ $\rightarrow \widetilde{\mathfrak{S}}_{2}^{*}=\mathfrak{S}_{2}^{*} \cup \widetilde{S}_{1}^{*} \cup \mathfrak{S}_{0}^{*} \operatorname{maps} \mathscr{M}$ and $\mathscr{N}$ to $\widetilde{S}_{2}^{*}, \mathscr{B}_{a}$ and $\mathscr{B}_{b)}$ to $\mathfrak{S}_{1}^{*}$, and $\mathscr{C}_{a)}, \mathscr{C}_{b)}$ and $\mathscr{D}$ to $\mathfrak{S}_{0}^{*}$.

(9.3) Now we shall prove Theorem 8 by reductio ad absurdum. Assume that for a point $\eta$ in $\widetilde{\varsigma}_{2}^{*}$ the fibre $F=j^{-1}(\eta)$ has a positive dimension.

The open set $\mathscr{U}_{2}$ in Theorem 7 is a union of strata $\mathscr{M}, \mathscr{B}_{a)}$ and $\mathscr{C}_{a \text { a) }}$. Hence by virtue of Theorem 7 no generic points in $F$ correspond to stable curves of types $\mathrm{I}, \mathrm{III}_{a}$, and $\mathrm{IV}_{a}$.

It is easy to see that the isomorphism classes of curves of types $\mathrm{IV}_{b)}$ and $V$ are unique, i.e. $\mathscr{C}_{b}$, and $\mathscr{D}$ consist of only one point respectively. Hence the generic point of $F$ is contained in $\mathscr{N}$ or $\mathscr{B}_{b \text { ) }}$.

First assume that the generic point $\xi$ is in $\mathscr{N}$. Let $C$ be a stable curve corresponding to $\xi$. Then $C$ is a union of two elliptic curves $C_{1}$ and $C_{2}$. By translation on each component we may assume that the origins of $C_{1}$ and $C_{2}$ meet together. Hence $C$ is determined uniquely by $C_{1}$ and $C_{2}$. On the other hand since $\eta$ is in $\widetilde{S}_{2}^{*}$, we can identify $j$ and $\bar{j}$ near $\eta$. Therefore $\eta$ corresponds to the isomorphism class of $J(C)=$ 
$J\left(C_{1}\right) \times J\left(C_{2}\right)$. Clearly it determines $C_{1}$ and $C_{2}$, hence $C$. Hence the assumption is impossible.

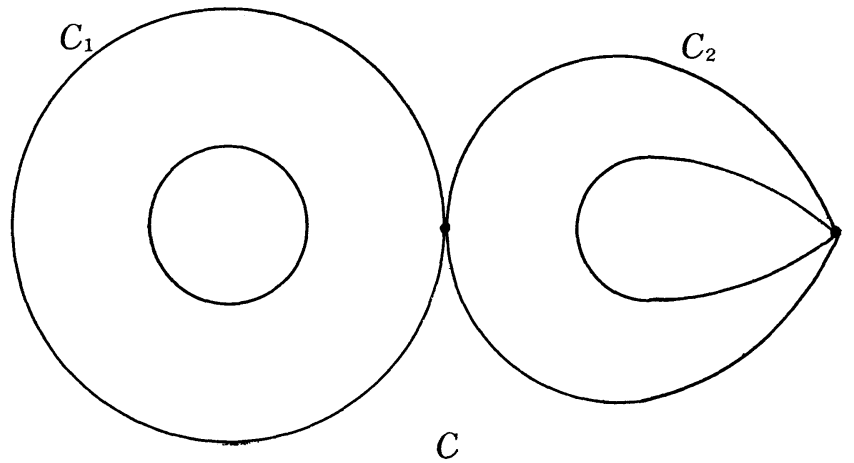

Now assume that the generic point $\xi$ of $F$ is in $\mathscr{B}_{b}$. Let $C$ be a stable curve corresponding to $\xi$. Then $C$ is a union of an elliptic curve $C_{1}$ and a rational curve $C_{2}$ with one double point. Since only one point in $C_{1}$ is specified and only three points in the normalization of $C_{2}$ are specified, the isomorphism class of $C$ is determined only by that of $C_{1}$. On the other hand since $J(\tilde{C})=C_{1}, \eta$ corresponds to the isomorphism class of $C_{1}$ in $\Im_{1}^{*}$, hence $\xi$ is uniquely determined by $\eta$, which again contradicts the assumption. q.e.d. of Theorem 8.

(9.4) We shall also give the explicit correspondence of $j$ in case of $g=2$ with the generalized period matrices (7.1). The proof is already done or easy, so we omit it. We shall use the notations in (8.2).

A) The case of type I. $g^{\prime}=2, g^{\prime \prime}=0$. In this case $j$ maps the isomorphism class of $C$ to the residue class of the period matrix $\Omega_{C}$ of $C$ in $\widetilde{S}_{2}^{*}$.

B) The case of type II. $g^{\prime}=2, g^{\prime \prime}=0$. Let $C$ be a stable curve with the irreducible components $C_{1}$ and $C_{2}$. Let $\tau_{1}$ and $\tau_{2}$ be the respective periods of $C_{1}$ and $C_{2}$. Then $j$ maps the point in $\mathscr{S}_{2}$ corresponding to $C$ to $\left(\begin{array}{cc}\tau_{1} & 0 \\ 0 & \tau_{2}\end{array}\right) \bmod . S p(2, Z)$ in $\widetilde{S}_{2}^{*}$.

C) The case of type $\mathrm{III}_{\mathrm{a}} \cdot g^{\prime}=1, g^{\prime \prime}=1$. Let $C$ be a stable curve of type $\mathrm{III}_{a}$, with normalization $\tilde{C}$ which is an elliptic curve with period $\tau$. Let $x, y$ be the points in $\tilde{C}$ corresponding to the unique double point of $C$. Then $j$ maps the point in $\mathscr{S}_{2}$ corresponding to $C$ to the point $(A, D, E)=(\tau, y-x, 0) \bmod . S p(2, Z)$.

D) The case of type $\mathrm{III}_{\mathrm{b})} \cdot g^{\prime}=1, g^{\prime \prime}=1$. Let $C$ be a stable curve 
of type $\mathrm{III}_{\mathrm{b}}$. It is a union of an elliptic curve $C_{1}$ with period $\tau$ and a rational curve with a double point. Then $j$ maps the point in $\mathscr{S}_{2}$ corresponding to $C$ to the point $(A, D, E)=(\tau, 0,0) \bmod . S p(2, Z)$.

E) The case of type $\mathrm{IV}_{\mathrm{a})} . \quad g^{\prime}=0, g^{\prime \prime}=2$. Let $C$ be a stable curve of type $\mathrm{IV}_{\mathrm{a}}$ with double points $z_{1}$ and $z_{2}$. Denote by $x_{i}, y_{i}$ the points in the normalization $\tilde{C}$ of $C$ corresponding to $z_{i}$. With a uniformizing parameter $z$ in $\tilde{C}$, we may assume $z\left(x_{1}\right)=0, z\left(y_{1}\right)=\infty$ and $z\left(y_{2}\right)=1$. Then $j$ maps the point in $\mathscr{S}_{2}$ corresponding to $C$ to the point $E=\left(E_{12}, E_{13}, E_{23}\right)$ $=\left(z\left(x_{2}\right), 0,0\right) \bmod . S p(2, Z)$.

F) The case of type $I \mathrm{~V}_{\mathrm{b})} . g^{\prime}=0, g^{\prime \prime}=2$. As we have remarked in the proof of Theorem 8, the isomorphism class of type $I_{\mathrm{b}}$ is unique. The point in $\mathscr{S}_{2}$ corresponding to it is mapped by $j$ to the point $E=$ $\left(E_{12}, E_{13}, E_{23}\right)=(1,0,0) \bmod . S p(2, Z)$.

G) The case of type V. $g^{\prime}=0, g^{\prime \prime}=2$. The isomorphism class of curves of type $\mathrm{V}$ is also unique, and the point in $\mathscr{S}_{2}$ corresponding to it is mapped by $j$ to the point $E=\left(E_{12}, E_{13}, E_{23}\right)=(0,0,0) \bmod . S p(2, Z)$.

\section{Addendum}

The proof of Theorem 4 is not complete. We need the following claim:

(*) for every point $s \in S$, when we express the period map of $\pi$ near $s$ as (4.8.2), there is a central cone $C_{\sigma}$ in $\mathfrak{Y}_{g}$ " containing "all" $B_{i, 0}$ where $B_{i, 0}=\left(b_{i, k s}\right)_{g^{\prime}<i, s \leq g^{\prime \prime}}$ (cf. (6.5)).

In case of $g \leq 6$ this claim holds for all families of stable curves. (It can be seen by direct calculation.) It seems true that it does hold in general.

The above claim being local, Theorem 4 is true for families of planar stable curves, for example. Hence the incompleteness of the proof of Theorem 4 gives no effect to the other theorems.

The author wishes to thank Professor P. Deligne who kindly pointed out my mistake of proof.

\section{REFERENCES}

[1] Artin, M.: On the solutions of analytic equations, Invent. math., Vol. 5 (1968), 277-291.

[2] Baily, W. L. Jr.: On compactifications of orbit spaces of arithmetic discontinuous groups acting on bounded symmetric domains, Algebraic Groups and Discontinuous Groups, Proc. Symp. Pure Math., Vol. 9, A.M.S., 1966, 281-295.

[ 3 ] Clemens, C. H. et al.: Seminar on degeneration of algebraic varieties, Institute for Advanced Study, Princeton, 1969-70. 
[ 4 ] Deligne, P. and Mumford, D.: The irreducibility of the space of curves of given genus, Volume Dédié au Professeur Oscar Zariski, Publ. math. I.H.E.S., No.36, Paris, 1969, 75-110.

[5] Grauert, H.: Ein Theorem der analytischen Garbentheorie und die Modulräume komplexer Strukturen, Publ. math. I.H.E.S., No. 5, Paris, 1960.

[6] Grothendieck, A. and Dieudonné, J.: Éléments de géométrie algébrique, Publ. math. I.H.E.S., Paris, 1960ff.

[ 7 ] Hurwitz, A. and Courant, R.: Vorlesungen über allgemeine Funktionentheorie und elliptische Funktionen, 4. Auflage, Springer-Verlag, Berlin, 1964.

[8] Igusa, J.: A desingularization problem in the theory of Siegel modular functions, Math. Ann., Bd. 168 (1967), 228-260.

[9] Knudsen, F. and Mumford, D.: to appear.

[10] Kobayashi, S. and Ochiai, T.: Satake compactification and the great Picard theorem, J. Math. Soc. Japan, Vol. 23 (1971), 340-350.

[11] Koecher, M.: Beiträge zu einer Reduktionstheorie in Positivitätsbereichen, I., Math. Ann., Bd. 141 (1960), 384-432.

[12] Koecher, M.: Ditto, II., Math. Ann., Bd. 144 (1961), 175-182.

[13] Nakamura, I.: On degeneration of abelian varieties, to appear.

[14] Namikawa, Y. and Ueno, K.: The complete classification of fibres in pencils of curves of genus two, Manuscripta math., Vol. 9 (1973), 143-186.

[15] — : On fibres in families of curves of genus two, I, Singular fibres of elliptic type, Number Theory, Algebraic Geometry and Commutative Algebra (in honor of Yasuo Akizuki), Kinokuniya, Tokyo, 1973, 297-317; II.: to appear.

[16] Popp, H.: On moduli of algebraic varieties, II., to appear.

[17* Ramis, J. P. and Ruget, G.: Complexe dualisant et théorème de dualité en géométrie analytique complexe, Publ. math. I.H.E.S., No. 38, Paris, 1970, 77-91.

[18] Satake, I.: On Siegel's modular functions, Proc. Internat. Symp. on Alg. Number Theory, Tokyo and Nikko, 1955, 107-129.

[19] ㄴ: On the compactification of the Siegel space, J. Indian Math. Soc., Vol. 20 (1956), 259-281.

[20] Séminaire H. Cartan: Fonctions automorphes, 10e année, 1957-58 Secrétariat math., Paris, 1958.

[21] Séminaire H. Cartan: Familles d'espaces complexes et fondements de la géométrie analytique, 13e année, 1960-61, 2e éd., Secrétariat math., Paris, 1962.

[22] Serre, J. P.: Géométrie algébrique et géométrie analytique, Ann. Inst. Fourier, t. $6(1955-56), 1-42$.

[23] —_: Groupes algébriques et corps de classes, Actualités scientifiques et industrielles, Hermann, Paris, 1959.

[24] Seshadri, C. S.: Quotient spaces modulo reductive algebraic groups, Ann. of Math., Vol. 95 (1972), 511-556.

[25] Siegel, C. L.: Symplectic geometry, Academic Press, New York, 1964; Gesammelte Abhandlungen, Springer, Berlin, 1966, Bd. 2, 274-360.

[26] Venkov, B. A.: On the reduction of positive quadratic forms (Russian), Izvest. Acad. Nauk. USSR, Vol. 4 (1940), 37-52.

[27] Voronoi, G.: Nouvelles applications des paramètres continus et théorie des formes quadratiques, I, II, III, J. Reine Angew. Math., Bd. 133 (1908), 97-178; Bd. 134 (1908), 198-287; Bd. 136 (1909), 67-181.

[28] Weil, A.: Zum Beweis des Torellischen Satzes, Nach. Akad. Wiss. Göttingen, Bd. 2 (1957), 33-53.

[29] Weyl, H.: Die Idee der Riemannschen Fläche, Teubner, Leipzig-Stuttgart, 1955.

Nagoya University

Universität Mannheim 